\title{
Anorexia and cachexia in cancer : a study with special emphasis on central tryptophan and serotonin metabolism
}

Citation for published version (APA):

von Meyenfeldt, M. F. (1983). Anorexia and cachexia in cancer : a study with special emphasis on central tryptophan and serotonin metabolism. [Doctoral Thesis, Maastricht University]. Rijksuniversiteit Limburg. https://doi.org/10.26481/dis.19830107mm

Document status and date:

Published: 01/01/1983

DOI:

10.26481/dis. $19830107 \mathrm{~mm}$

Document Version:

Publisher's PDF, also known as Version of record

\section{Please check the document version of this publication:}

- A submitted manuscript is the version of the article upon submission and before peer-review. There can be important differences between the submitted version and the official published version of record.

People interested in the research are advised to contact the author for the final version of the publication, or visit the DOI to the publisher's website.

- The final author version and the galley proof are versions of the publication after peer review.

- The final published version features the final layout of the paper including the volume, issue and page numbers.

Link to publication

\footnotetext{
General rights rights.

- You may freely distribute the URL identifying the publication in the public portal. please follow below link for the End User Agreement:

www.umlib.nl/taverne-license

Take down policy

If you believe that this document breaches copyright please contact us at:

repository@maastrichtuniversity.nl

providing details and we will investigate your claim.
}

Copyright and moral rights for the publications made accessible in the public portal are retained by the authors and/or other copyright owners and it is a condition of accessing publications that users recognise and abide by the legal requirements associated with these

- Users may download and print one copy of any publication from the public portal for the purpose of private study or research.

- You may not further distribute the material or use it for any profit-making activity or commercial gain

If the publication is distributed under the terms of Article $25 \mathrm{fa}$ of the Dutch Copyright Act, indicated by the "Taverne" license above, 


\section{ANOREXIA AND CACHEXIA IN CANCER}





\section{Anorexia and cachexia in cancer}

A study with special emphasis

on central tryptophan and serotonin metabolism

\section{Proefschrift}

ter verkrijging van de graad van doctor in de geneeskunde aan de Rijksuniversiteit Limburg te Maastricht, op gezag van de Rector Magnificus Prof. Dr. H.C. Hemker, volgens besluit van het College van Dekanen in het openbaar te verdedigen in de aula van de universiteit op vrijdag 7 januari 1983, des namiddags te vier uur

door

Maarten Frederik von Meyenfeldt

geboren te Makassar 
Promotores: Prof. Dr. J.M. Greep

Prof. J.E. Fischer, M.D.

Referenten: Dr. P.B. Soeters

Prof. Dr. H.A.J. Struyker-Boudier

Prof. Dr. P. Visser

$\mathbb{1} \mathbb{8}$

krips rapro meppel

The experimental studies reported in this thesis were performed at the Surgical Physiology Laboratory. Department of Surgery, University of Cincinnati Medical Center, Cincinnati, OH., U.S.A.

Financial support for the publication of this thesis was received from

Wander Pharma, Div. of Sandoz B.V., Uden

NV. Verenigde Bedrijven Nutricia Zoetermeer.

Jan Dekkerstichting en Dr. Ludgardine Bouwmanstichting 
Despite nearly half a century of intense and clinically relevant research in the field of nutrition the typical physician still considers malnutrition to be synonymous with a potbellied, holloweyed Southeast Asian child...

It has now become clear that starvation, malnutrition, or both and the inability of patients to receive adequate nourishment during the course of severe illness, have far-reaching immunological and functional effects:.

Josef E. Fischer

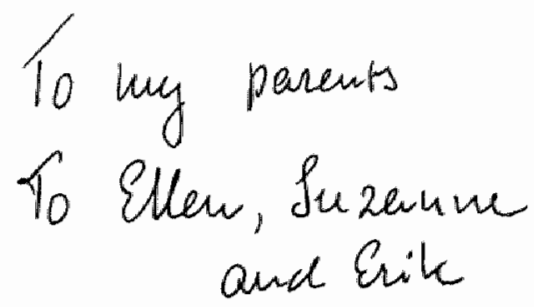





\section{Contents}

Abbreviations $X I I$

\section{PART I: INTRODUCTION}

\section{Chapter 1}

\section{Background 3}

1.1. General introduction to the problem of cancer anorexia 3

1.2. Proposed mechanisms for the regulation of food intake 3

1.2.1. A central regulatory system 3

1.2.2. An alimentary tract regulation 4

1.2.3. A thermostatic regulation 4

1.2.4. A lipostatic regulation 5

1.2.5. A glucostatic regulation 5

1.3. The influence of amino acids on the neuroregulation of food intake 5

1.4. Tryptophan, brain serotonin and food intake 6

1.5. Tryptophan, brain serotonin and food intake in cancer 9

\section{Chapter 2}

Introduction to and methodological considerations regarding the experiments in this thesis 11

2.1. Purpose 11

2.2. Outline of the experimental work 11

2.2.1. Experiments, designed to investigate correlations between altered brain serotonin metabolism and cancer anorexia (part II) 11

2.2.2. Efforts to manipulate the entry of tryptophan into the brain and the indoleamine system in cancer anorexia (part III) 12

2.3. Methodologicall considerations 12

2.3.1. Animals 12

2.3.2. Tumor models 12

2.3.3. Biochemical assays 73

2.3.4. Statistics 14 


\section{Chapter 3}

Replication of tryptophan and serotonin system changes in and further definition of the Walker 256 carcino sarcoma model of cancer anorexia

3.1. Introduction 17

3.2. Materials and methods 17

3.3. Results 18

3.4. Discussion 26

3.5. Conclusions 27

\section{Chapter 4}

Developmentall changes in central serotonergic activity during growth of a $W_{256}$ carcino sarcoma 29

4.1. Introduction 29

4.2. Materials and methods 31

4.3. Riesults 31

4.4. Discussion 31

4.5. Canclusions 32

\section{Chapter 5}

Generality of observed tryptophan and indoleamine system derangements in anorectic tumor bearing animals 33

5.1. Introduction 33

5.2. Materials and methods 33

5.3. Results 34

5.4. Discussion 38

5.5. Conclusions 40

\section{Chapter 6}

Regional brain serotonergic activity in tumor induced anorexia. studied in two tumor models 41

6.1. Introduction 41

6.2. Materials and methods 41

6.3. Results 42

6.4. Discussion 46

6.5. Conclusion 47 


\section{Chapter 7}

Summary of the results and conclusions of the experiments described in part ll 49

7.1. Tumor models 49

7.1.1. Walker 256 carcino sarcoma 49

7.1.2. Methylcholanthrene induced sarcoma 49

7.2. Serotonin 50

7.2.1. Plasma tryptophan 50

7.2.2. Blood-brain barrier transport 50

7.2.3. Brain tryptophan, serotonin and 5-hydroxyindoleacetic acid 50

7.2.4. Conclusion 51

7.3. Catecholamines 51

Part III: EFFORTS TO MANIPULATE THE ENTRY OF TRYPTOPHAN INTO THE BRAIN AND THE INDOLEAMINE SYSTEM IN CANCER ANOREXIA

\section{Chapter 8}

The effect of branched chain amino acids enriched diets on food intake of tumor bearing animals 55

8.1. Introduction 55

8.2. Materials and methods 55

8.3. Results 56

8.4. Discussion 60

8.5. Conclusions 61

\section{Chapter 9}

The effect of parachlorophenylalanine on serotonin synthesis and food intake in cancer anorexía 63

9.1. Introduction 63

9.2. Materials and methods 63

9.3. Riesullts 64

9.4. Discussion 68

9.5. Conciusions 68 


\section{Chapter 10}

The effect of methysergide on food intake in anorectic

cancer bearing rats 69

10.1. Introduction 69

10.2. Materials and methods 69

10.3. Results 71

10.4. Discussion 72

10.5. Conclusions 73

\section{Chapter 11}

The effect of 5.7-dihydroxytryptamine on serotonin metabolism and food intake in cancer induced anorexia 75

11.1. Introduction 75

11.2. Materials and methods 75

11.3. Results 76

11.4. Discussion 77

11.5. Conclusions 78

\section{Chapter 12}

The effect of intrahypothalamic administration of norepinephrine with and without manipulation of the serotonin system on food intake in tumor bearing animals 79

12.1. Introduction 79

12.2. Materials and methods 79

12.3. Results 81

12.4. Discussion 82

12.5. Conclusions 82

\section{Chapter 13}

Summary of the results and conclusions of the experiments described in part Ill 85

13.1. Tryptophan and serotonin 85 13.1.1. Effort to lower brain tipyptophan 85

13.1.2. Effect of serotonin synthesis inhibition 85

13.1.3. Effect of postsynaptic receptor blackade 85

13.1.4. Effect of the neurotoxic drug 5,7-dihydroxytryptamine 86

13.1.5. Effect of central chemical stimulation 86

13.1.6. Conclusion 86

13.2. Catecholamines 87 
PART IV: CONCLUDING REFLECTIONS

\section{Chapter 14}

Cancer anorexia 91

14.1. Plasma tryptophan and cancer anorexia 91

14.2. Brain serotonin and cancer anorexia 92

14.2.1. Serotonin receptors 92

14.2.2. False neurotransmitter formation 93

14.2.3. No specific serotonin involvement 93

14.2.4. Conclusion 94

14.3. Tyrosine, catecholamines and cancer anorexia 94

14.3.1. Norepinephrine 94

14.3.2. Dopamine 95

14.3.3. Conclusion 95

\section{Chapter 15}

Cancer anorexia and cachexia 97

Summary 99

Samenvatting 103

References 107

Acknowledgements 117

Curriculum vitae 119 


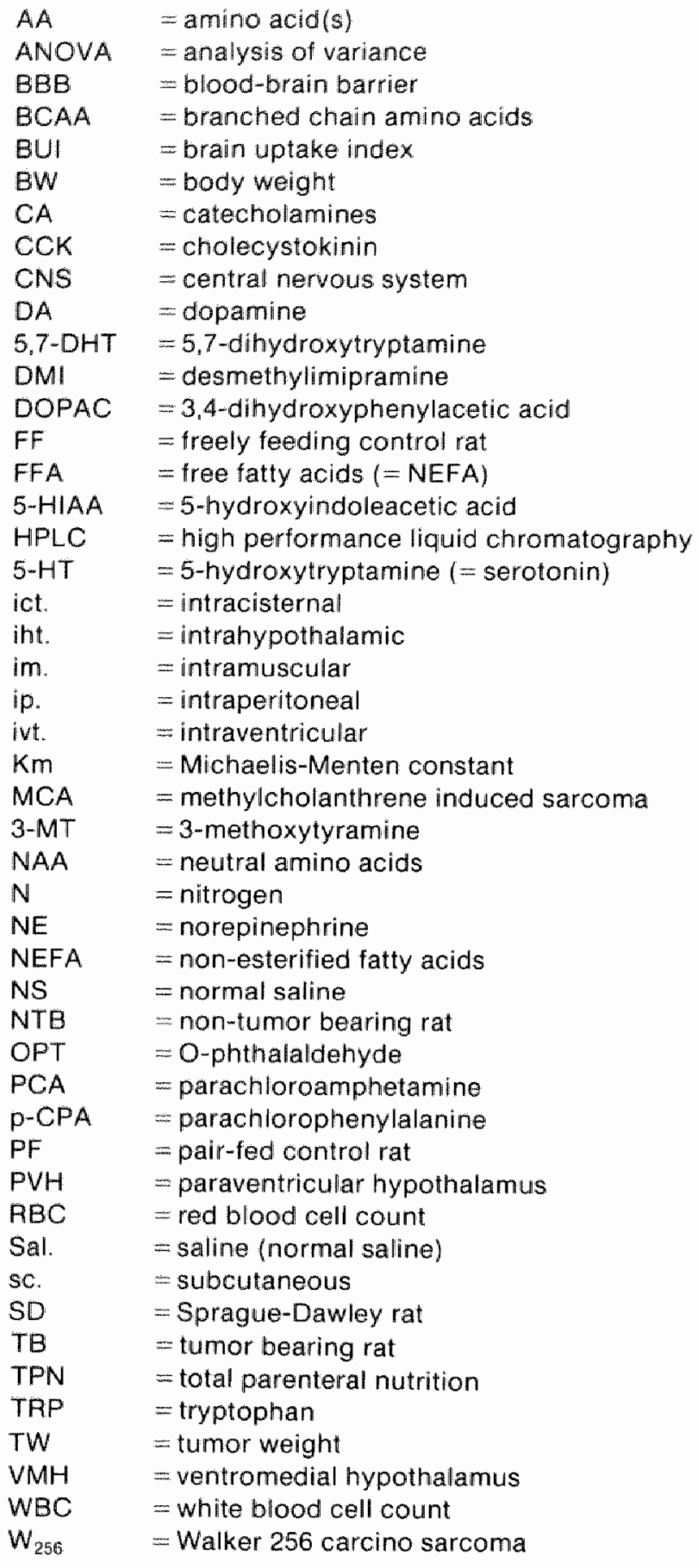


Part I

INTRODUCTION 



\section{Chapter 1}

\section{Background}

\subsection{General introduction to the problem of cancer anorexia}

Anorexia is a common phenomenon in cancer disease, often believed to be present in early stages (Theologides, 1974). The incidence of this phenomenon is not known however, and no quantification of the problem has been attempted. This absence of knowledge results in part because of the difficulty to isolate other influences on food intake behavior, such as psychological and emotional factors. Therapeutic regimens also disturb the assessment of the relationship of the anorexia to cancer (Theologides, 1972). Another reason for this lack of understanding is that major aspects of the normal physiology of hunger and satiety remain hypothetical, thus further complicating our understanding of the mechanisms of anorexia associated with disease (Theologides, 1976). Finally, the quantitative relationship between anorexila and the development of cancer cachexia is not well understood (Theologides, 1974; Lundholm, 1980) and consequently anorexia not well recognized as a clinical problem. The use of elaborated intravenous (hyper-) alimentation techniques has contributed considerably to the understanding of the anorexia-cachexia syndrome. However these techniques have also clouded the understanding of complicated tumor-host relationships by suggesting that simple suppletion with calories and nitrogen would result in restoration of the nutritional integrity of the tumor-host. Since in prospective randomized trials hyperalimentation did not appear to normallize cachexia effects in cancer disease (Brennan, 1981), even more attention may be directed towards characterization of the metabolic events that take place in the host and between the host and the tumor. Assessment of these problems should include a search for the cause of cancer induced anorexia.

\subsection{Proposed mechanisms for the regulation of food intake}

Many theories hawe been proposed for the regulation of food intake, and subsequently their potential role in the development of cancer anorexia were hypothesized and investigated. Some of these theories are briefly reviewed below.

\subsubsection{A central regulatory system}

A system in which the hypothalamus plays a major role for hunger and satiety has been proposed by Hetherington (1940). Brobeck (1943) and Anand and Brobeck (1951). The effects of lesions to certain parts of the hypothalamus on feeding behavior led to the concept of a 'satiety" center, located in the ventromedial nucleus, and a 'feeding' center. located in the lateral hypothalamus. Subsequent research has suggested that this interpretation is too simple (Grossman, 1980). In addition to these lesion studies, the association of anatomical structures with neurochemical determinations has added a functional aspect to this concept. Indeed did Ungerstedt (1971) describe a map of monoamine pathways in the rat brain, that provided possibilities for pharmacological 
manipulations. Thus, studies by Leibowitz (1976) provided evidence that a-adrenergic receptors in the medial paraventricular hypothalamus may mediale feeding stimulation. whereas $\beta$-adrenergic and dopaminergic receptors. located in the antero-latera! hypothalamic region, may mediate feeding inhibition. Both receptor systems seemed to be part of different pathways that seemed to originate in the midbrain and pass through hypothalamic regions before innervating other brain ares.

Studies by Baillie (1965) and Morrison (1968) in Walker 256 carcino sarcoma bearing rats have shown that such a regulatory system is not influenced by the presence of a tumor. Thus, Baillie demonstrated anorexia occurring at the same time and to the same severity in Walker 256 tumor bearing rats previously rendered hyperphagic by destruction of the ventromedial hypothalamus, as compared to untreated TB controls. Lesions to the lateral hypothalamus induced true aphagia and adipsia, suggesting normal functioning of the lateral area.

\subsubsection{An alimentary tract regulation}

Such a system would inwolve a variety of mechanisms, on different levels to influence feeding. The senses: sight, taste and smell, may trigger brain mechanisms that will modulate feeding (Wooley, 1975).

Ingestion of food may influence the central nervous system through distension of the stomach, as suggested by increased electrical activity in hypothalamic "satiety" centers after balloon inflation induced gastric distension (Sharma, 1961). Also, the normally observed inhibition of gastric contractions following a meal, is not seen after lesions to these 'satiety' centers (Sharma, 1961). Furthermore, compensatory drinking occurred when a certain volume of stomach contents was withdrawn or allowed to pass to the duodenum in rats with inflatable pyloric cuffs (Deutsch, 1978).

A humoral factor, released secondary to food intake, may also signal satiety. Flemming (1969) found decreased food intake in one rat of a parabiotic pair, if its partner was fed one hour before. This humoral factor seemed unrelated to blood glucose ievels, or to hypothalamic regulation of food intake.

Finally, the release of peptides, such as cholecystokinin (CCK), have been implicated in the control of feeding. Gibbs (1973) described decreased food intake in rats following administration of CCK. His results were confirmed by Della-Fera (1979), who observed decreased food intake in a dose-related manner after intraventricular administration of CCK octapeptide in sheep. McCaleb (1980) described CCK attenuating or even blocking feeding responses to intrahypothalamically administered morepinephrine.

Alterations of these regulatory systems, related to cancer anorexia, have been described DeWijs (1978) reported altered taste sensations in cancer patients. In addition, the acquisition of learned food aversions has been described in tumor-bearing animals (Bernstein, 1980). Thus, appetite loss in cancer patients may be due not only to the direct effects of tumor treatments with unpleasant side effects, but to aversions learned as a consequence of the association of foods with these side effects.

The mediation of cancer anorexia through a 'humoral' factor has been hypothesized. without concrete evidence for its existence however (Theologides, 1974).

It has been demonstrated in the Surgical Physiology laboratory (Univ. of Cincinnati) that cholecystokinin is not anymore potent in decreasing food intake un tumor bearing animals as it is in non-tumor bearing controls ( $v$. Lammeren, 1982

\subsubsection{A thermostatic regullation}

Strominger and Brobeck (1953) suggested that the heat resulting from the specific dynamic action of food regulates food intake. Thus, in a cold environment, animals would eat to keep 
warm, while they would stop eating to prevent hyperthermia. Anorectic tumor-bearing animals "placed in a cold environment, increased their oral intake lemponarily, but the anorexia reappeared subsequently, thus suggesting that the need for temperature homeostasis was stronger than the anorexigenic action, elicited by the presence of a tumor (Stevenson, 1961). No causative relationship however has been demonstrated.

\subsubsection{A lipostatic regulation}

Body lipids, and more precisely free fatty acids and glycerol have been described in the regulation of food intake (Oomura, 1976). Rapid alterations during the day of the free fatty acid-levels, however, suggest that these compounds do not generally exert significant control functions. Glycerol is not readily being transported across the blood brain bartier, and may act through peripheral sensors.

However, it is not clear what importance these factors have in the development of cancer anorexia. Liebelt (1971) observed anorexia in genetically-obese and gold-thio-glucoseobese tumor bearing mice, but not in normal or lean hosts, although both groups exhibited approximately the same percentage decrease in lipid stores, and consequent hyperlipidemia.

\subsubsection{A glucostatic regulation}

In an effort to explain the interaction between the ingestion of food and the signalling of satiety to feeding control areas of the brain, Mayer (1955) proposed the presence of glucoreceptors in the hypothalamus. These would be sensitive to "effective blood sugar levels', that is, the rate of glucose utilization. In support of this hypothesis Anand (1964) reported differentially influenced hypothalamic electric spike activity after the iv.

administration of glucose, insulin, or a combination of both. Thus, spike frequency recorded from the 'satiety center neurons' increased, while that of 'feeding center neurons' decreased. Neuronal activity correlated best with arterio-venous glucose differences, rather than with blood glucose levels per se.

It is widely accepted that glucose metabolism is altered in cancer disease (Brennan, 1977). Most prominent is a relative insulin resistance (Lundholm, 1978 ${ }^{\circ}$ ). How this decreased glucose utilization, in the presence of increased blood sugar levels, could account for mediation of the observed anorexia in cancer patients, is not clear.

A hypothetical aminostatic regulatory system of food intake control will be discussed separately in the next section.

\subsection{The influence of amino acids on the neuroregulation of food intake}

The influence of amino acids on food intake has been known for a long time. In 1916 (b) Osborne and Mendel wrote: 'We have frequently noted that when the protein concentration of the food becomes very low the animals do not eat satisfactorily. . . In the same year they published results illustrating the effect of an amino acid deficiency on food intake (Osborme, $1916^{\circ}$ ). They fed increasing supplements of lysine with a gliadin diet to rats and found increasing weight gain and increasing quantities of food ingested. They continued, however, to describe pairmeeding experiments and detailed growth curves and concluded that the depression in food intake was a secondary effect, resulting from poor growth. It did not become apparent until the early 1930's, when Rose began his work on amino acid requirements, that the idea was set forth clearly that an amino acid deficiency was synonymous with a depression in food intake and with problems with the acceptability of 
the diet. Thus, Rose (1931) wrote: 'Our experience with other types of deficiencies involving the nitrogenous portion of the ration has taught us to expect a marked failure in appetite when the diet is completely devoid of an essential component".

Although it has since been observed consistently that feeding an amino acid deficient diet results in a rapid depression of food intake. little work was done on the mechanism of the depression of food intake until the last two decades. Only after reconsideration of the brain as an autonomous organ, that removes from the circulation the substances it requires. oblivious to the dietary history or metabolic state of its host, was it realized, that the brain is susceptible to dietary influences (Curzon, 1978; Wurtman, 1979). Thus, the mervous system is intricately involved in the food intake regulation by plasma amino acids.

In their effort to correlate changes in amino acid patterns to brain areas, Rogers and Leung (1973) described four categories of amino acid patterns to which the rat responds by changes in feeding:

\section{Amino acid imbalance}

This condition has been reported to result in food intake depression and altered dietary choice (Harper, 1965). In addition Leung (1969) observed changes in the pattern of eating of rats fed an isoleucine imbalanced diet. The rat would decrease the number of meals per day after exposure to the test diet, increase the meal size after an adlaptation to the diet, thus eating a pre-test $24 \mathrm{hr}$ amount of food, and overeat when the deficiency was corrected.

\section{Amino acid deficiency}

An amino acid deficiency would arise when a diet completely devoid of an essential amino acid was fed. A diet devoid of isoleucine induced marked decreased food intake of a rat by decreasing meal sizes. Adlaptation to the diet consisted of frequent small meals, even during the light period of the day, with small effects on the total daily intake. This observation suggests that the rat was continually hungry, but refused to eat much of the deficient diet at any one time. Addition of the deficient component to the test diet induced immediate restoration of normal food intake (Leung, 1969).

\section{High protein diet}

Depression of food intake and subsequent metabolic adaptation and restoration of food intake caused by the feeding of a high protein diet have been described (Anderson, 1968). It appeared that the rat fed a high protein diet would decrease the meal sizes. After adaptation, meal frequency was decreased, but meal sizes were increased. This adaptation resulted in restoration of pre-test $24 \mathrm{hr}$ period food intake values. Decreasing the protein component of the diet resulted in marked overeating.

\section{Excess of one amino acid}

Most amino acids will depress food intake and growth if only given at high enough levels. The essential amino acids, and especially methionine, tryptophan and leucine, exert the greatest depression. Rats, fed a leucine enriched diet, would decrease the number of meals but not the meal sizes, thereby reducing their $24 \mathrm{hr}$ consumption. This $24 \mathrm{hr}$ consumption remained reduced after adaptation in the presence of decreased meal sizes and increased meal frequencies.

How the above described diet-induced amino acid patterns would exert their effect on food intake, is not known. It has been postulated that the excess or deficit amino acid in plasma may be the one common unique metabolic event, influencing some receptor to respond and decrease food intake. In an effort to determine what neurostructures would be involved in mediating the amino acid pattern induced changes in feeding. Rogers and Leung (1973) could reverse amino acid deficiency induced anorexia by prepyriform cortical lesions, and imbalance induced anorexia by medial amygdala and prepyritorm cortical lesions. They 
could not locate, however, brain areas which, when destroyed by electrolytic lesions would prevent the food intake depression that occurs when an animal is fed an excess of one or all of the amino acids.

Not only diet manipulations induced plasma amino acid patterns may be of importance when considering an amino acid regulation of food intake. Disease states known to be associated (clinically) with anorexia, are correlated with altered plasma amino acid, and also brain amino acid patterns: sepsis (Jeppsson, 1980), uremia (Jeppsson, 1982), respiratory and metabolic acidosis (Jeppsson, 1980') and hepatic failure (Rosen, 1977; Soeters, 1979). In none of these reports, however, was the relationship between these amino acid patterns and food intake investigated.

In recent years the wiew has gained adherence that nutrients alter plasma lewels of amino acids, which may induce alterations in brain neurotransmitter synthesis. The amino acid tryptophan has been widely studied in this respect (Fernstrom, 1981).

\subsection{Tryptophan, brain serotonin and food intake.}

In recent years dietary influences on precursor availability and neurotransmitter formation have been studied, and numerous reports have been published with special reference to tryptophan (TRP) and serotonin (5-HT) synthesis, and their possible role in the physiology of the control of feeding. Thus, these studies hypothesize the following model of TRP and 5-HT metabolism in the control of food intake.

Ingestion of a meal results in alterations of plasma amino acid concentrations, and consequently of TRP (Fernstrom, 1978). This amino acid is unique, in that it is distributed in serum between two pools: approximately $25 \%$ circulates free, the remainder binds to serum albumin (Mc Menamy 1958, 1961).

Curzon (1973) demonstrated in vitro that a rise of non-esterified fatty acid concentrations (NEFA) induced increased levels of the unbound TRP fraction, presumably because NEFA compete with TRP for the same bind ing site on albumin. This concept seems to be confirmed by Madras (1974), who found increasing levels of plasma free TRP with increments in plasma free fatty acids (FFA). When FFA were increased by six fold no further raise of plasma free TRP could be detected, but a fall in plasma total TRP was noted. This observation suggests that unbound TRP can be present in plasma to a certain maximum concentration and that further displacement of this amino acid from its binding site on albumin, results in its disappearance by the same amount from plasma, presumably by degradation in the liver (Rodwell. 1977)

In addition to the NEFA influence on TRP binding to albumin, Yuwiler (1977) observed decreased TRP binding to albumin as plasma $\mathrm{pH}$-values increased.

The importance of either tryptophan fraction for transport of this amino acid into the brain is also relative to the presence of other large neutral amino acids (NAA: Valine, Methionine, isoleucine Leucine, Tyrosine. Phenylalanine and Histidine). This interaction has been the subject of extensive work, generated and published by Oldendorf (1971), Yuwiller (1977) and Pardridge (1977, 1979 $\left.{ }^{\mathrm{a}+\mathrm{b}}\right)$ and summarized below:

Tryptophan uptake into the brain is concentration dependent ${ }_{\text {is greater from albumin free }}$ solutions than from solutions containing this plasma protein, is subject to contributions from both 'free' and 'bound' fractions, and finally, is under strong influence of amino acid competition at the large neutral amino acid blood-brain barrier (BBB) carrier site.

Because in their experimental design plasma free TRP concentrations could not account for the measured brain concentrations, it was hypothesized that a considerable portion of TRP would be stripped from albumin during passage through the capillary plexus. Their data indicate, that the capacity/affinity ratio is much higher for BBB-binding and transport of 


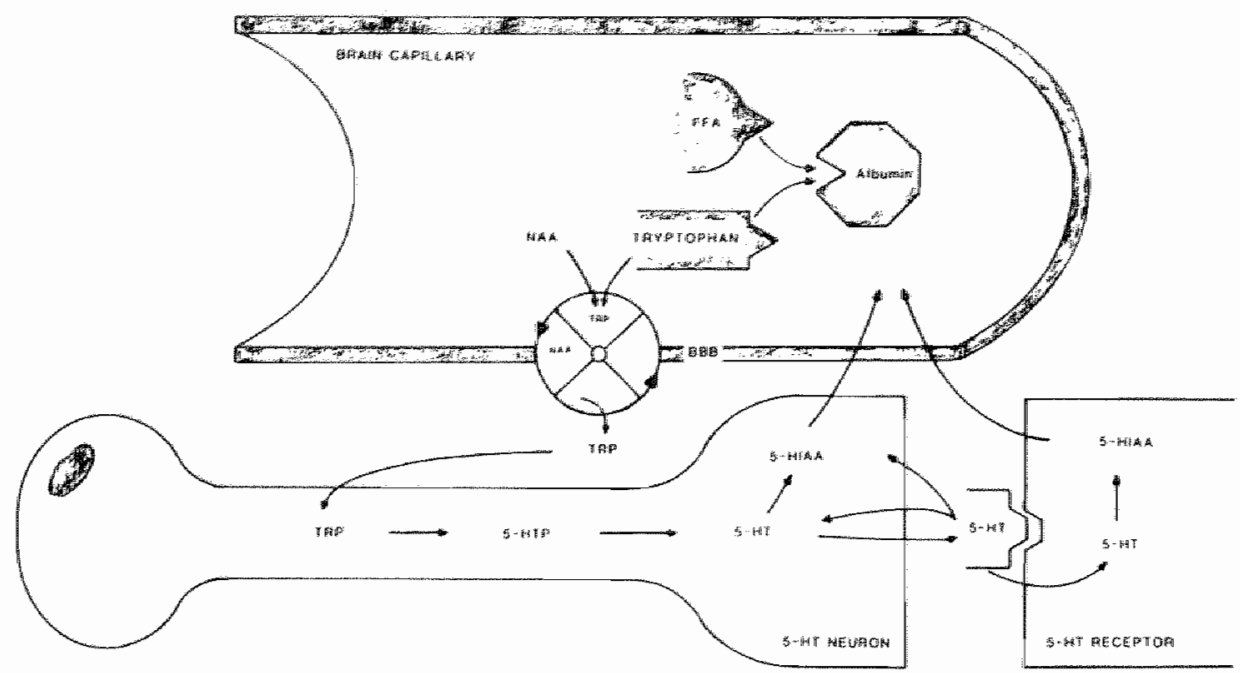

Fig. 1.4.-1 Tryptophan uptake into the brain and serotonin synthesis. The figure shows factors influencing tryptophan (TRP) avalability for blood-brain barrier (BBB) transport, and precursor dependence of serotonin (5-HT) synthesis.

Free fatty acids (FFA) compete with TRP for binding to albumin. Furthermore, neutral amino acids (NAA) compete with TRP for binding to the NAA carrier system in the BBB. Within 5-HT neurons TRP is hydroxylated to 5-hydroxylryptophan (5-HTP): 5-HTP is decarboxylated to serotonin.

Monoamine oxidase initiates the catabolism of 5-HT to 5-hydroxyindoleacetic acid (5-HIAA)

TRP, relative to albumin binding of the amino acid (Pardridge, 1979'). The mechanism underlying this phenomenon remains hypothetical.

In contrast to the above theory are reports that describe increased brain TRP concentrations after a $24 \mathrm{hr}$ food deprivation period in the presence of lowered plasma total TRP levels, and an increased plasma free TRP fraction (Taglliamonte, 1973; Perez-Cruet, 1974; Curzon; 1975; Young, 1976). Which one of the factors contributes most to TRP transport into the brain appears to depend on the specific circumstances of observation: plasma total TRP concentration. TRP binding to albumin, or concentrations of the competing neutral amino acids (Yuwiler, 1977).

The Michaelis-Menten constant $(\mathrm{Km})$ is the term for the substrate concentration that produces half maximal velocity of an enzymatic reaction. Thus, the $\mathrm{Km}$ of the rate limiting enzymatic step in the symthesis of the monoamine neurotransmitter serotonin "tryptophan hydroxylase, is also important with respect to the synthesis rate of serotonin. While this constant is equal to, or greater than physiological intracellular concentrations of the substrate amino acid, tryptophan, precursor supply, viz. tryptophan, will influence the rate of the reaction, and consequently the BBB transport phase is rate affecting (Pardridge, 1979').

The hydroxylation step is followed by decarboxylation of 5-hydroxytryptophan to 5-hydroxytryptamine, the biologically active amine serotonin. Degradation within either preor postsynaptic neurons via 5-hydroxyindoleacetaldehyde yields 5-hydroxyindoleacetic acid (5-H|AA), which is cleared from the brain and excreted in the urine (Bender, 1978). With the above described knowledge, to which their work also basically contributed. Fernstrom and Wurtmans observations on dietary influences on brain TRP concentrations. and 5-HT synthesis, can thus be summarized and explained:

Ingestion of a carbohydrate meal increases plasma TRP levels, simultaneous to insulin induced reductions of the other neutral amino acids, predominantly the branched chain amino acids (Fernstrom, 1971). Thus competitive advantage for TRP would arise over the 
other neutral amino acids, resulting in increased brain TRP levels and increased 5-HT synthesis and degradation. In contrast, ingestion of a $18-240$ casein diet would not only result in plasma tryptophan elevations, but also in comparablie increments of the competing neutral amino acids, thus avolding a change in the competition at the blood brain barrier carrier site, and consequently in brain tryptophan concentrations (Fernstrom, 1972", 1979) Furthermone, starvation results in displacement of tryptophan from albumin binding sites by non esterified fatty acids, which increase as a result of lipolytic processes associated with lood deprivation. Consequently brain TRP levels, 5-HT synthesis and degradation increase (Curzon, 1972; Perez-Cruet, 1972).

The functional significance to the brain and body of this concept, that diet induces alterations in plasma and brain neutral amino acid levels and neurotransmitter synthesis, is becoming increasingly clear. Related to the control of feeding behavior, the weight of evidence suggests that brain 5-HT may function as a satiety signal. Thus, direct injection of serotonin into the lateral hypothalamus (Singer, 1971; Lehr, 1973), into the lateral wentricle (Kruk, 1973), or in the perifornical hypothalamus (Goldman, 1971) of rat brain elicits satiety. as does systemic (ip.) administration of the precursors tryptophan (Latham, 1979) and 5-hydroxytryptophan (Blundell, 1975, 1979).

The anorectic drugs fenfluramine (Garattini, 1975) and fluoxetine (Fuller, 1977) indirectly increase functional serotonin activity by increasing its release or blocking its synaptic reuptake respectively. Furthermore, systemic injection of the serotonin receptor stimulants, quipazine (Samanin, 1977) and Lilly 110-140 (Goudie, 1976) showed, that these are very potent anorectic agents in rats. Antagonism of CNS serotonergic systems has been reported to produce the opposite effects, and elicit eating. Thus, the serotonin receptior blockers methysergidle (Barrett, 1975) and cyproheptadine (Ghosh. 1973; Kruk, 1973; Clineschmidt, 1974 ) abolish the anorectic effect of serotonin agonists, as well as increase food intake in rats (Baxter, 1970). Intraventricular injection of the serotonin symthesis inhibitor" parachlorophenylalanine, has been reported to elicit hyperphagia in rats (Breisch, 1976). Additional qualitative information on serotonin function was reported by Ashley (1975)" suggesting an inverse relationship between the plasma tryptophan over neutral amino acids ratio to protein intake.

\subsection{Tryptophan, brain serotonin and food intake in cancer}

The concept that the presence of a tumor induces increased metabolic needs (Mider, 1948 ; Fenninger, 1954; Morrison, $1976^{b}$ ) and that there is no increase in energy intake, but rather an absolute decline in food intake (Mider. 1948) is suggestive for a dysfunction of CNS mechanisms, that normally control hunger and satiety.

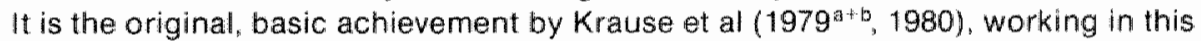
aboratory, to try to relate this notion to the accumulating knowledge regarding the inhibitory effects of serotonim on food intake behavior and thus to try to relate new basic physiological knowledge to circumstances of pathology.

Thus, working with an intramuscularly implanted Walker 256 carcino sarcoma in immature female Sprague-Dawley rats, Krause $\left(1979^{\text {a }}\right)$ observed significant anorexia in TB animals for at least 72 hr prior to decapitation on day 10 post tumor implantation.

In order to identify undernutrition induced changes that were not tumor specific, a pair fed (PF) control group matched in weight to the TB group, was included in their studies. This group was presented with the amount of chow eaten by their tumor bearing counterparts in the previous $24 \mathrm{hr}$. period.

Analysis of plasma amino acid levels indicated reduced aspartate, hydroxyproline, asparagine, glycine, citrulline and arginine levels as compared to the PF control group, while tyrosine was elevated, and total TRP was decreased as compared to freely feeding 
(FF) controls. Similar changes in the levels of these wo amino acids in PF animals suggested a nutritional etiology.

Ir addition, analysis of brain amino acid levels indicated significant, as compared to PF controls, decreases in taurine, citrulline, ornithine, and arginine, while leucine and TRP levels were elevated. Furthermore, brain 5-HIAA concentrations were significantly increased. Thus, brain TRP was increased in the presence of decreased plasma total TRP concentrations. Therefore those factors that are of importance to TRP penetration across the blood-brain barrier into the brain were next investigated.

- Albumin. Plasma albumin concentrations were decreased in TB-animals, presumably due to deposition of albumin in the tumor, and increased degradation relative to the increased albumin synthesis, resulting from the presence of a tumor (Lundholm, $1978^{\text {a. }}$ : Karlberg, 1980).

- Non-esterified fatty acids. Plasma free fatty acids were increased significantly as compared to the FF-control group. Abnormally increased lipid mobilization has been known to occur for some time (Mider, 1948) in tumor bearing animals, leading to increased plasma free fatty acid concentrations, albeit that the mechanism behind this phenomenon remains unknown (Lundholm, 1980).

- Plasma pH was unaltered in TB animals as compared to both control groups.

The above described observations were sufficient to explain the marked increments of plasma free TRP concentrations in Walker 256 TB animals: $200 \%$ of the values in FF controls.

- Competition by the other large neutral amino acids. Concentrations of these competing arnino acids (Valine, Methionine, Isoleucine, Leucine, Tyrosine, Phenylalanine, Histidine) were not different across the experimental groups.

Thus, changes observed sofar would result in a competitive advantage of TRP over the other $N_{A A}$ if the free TRP fraction were the more important one in determining TRP transport into the brain. This is not an unreasonable assumption (see part 1.3).

To exclude changes in activity in the blood-brain barrier neutrall amino acid transport system as another source of increased brain TRP concentrations, the brain uptake index (BUI) for TRP, as described by Oldendorf (1971), was assessed for each experimenta: group, and found to be unchanged.

On the basis of these observations Krause formulated the hypothesis, stating that altered TRP binding in plasma may lead to increased brain TRP levels, that in turn induce increased serotonin synthesis and degradation, thus contributing to a central mechanism of the anorexia observed in experimental cancer.

The experimental work to be described in this thesis started from this point. Thus, whereas the proposed hypothesis was based on changed peripheral TRP binding, that was investigated to a substantial degree, and that was assumed to be the main inductor of increased serotonergic activity in the brain, the most delicate feature of the hypothesis was that a causal relationship between cancer anorexia and central serotonergic activity remained to be proven. Therefore, experiments were designed to further detail correlations, thus providing additional circumstantial evidence, and to manipulate the serotonergic system, that would provide evidence for a causal relationship. 


\section{Chapter 2}

\section{Introduction to and methodological considerations regarding the experiments in this thesis}

\subsection{Purpose}

Experiments to be discussed in this thesis concern serotonergic influences on the development of cancer induced anorexia. As outlined in he final part of chapter 1 the hypothesis to be tested concerns altered peripheral TRP metabolism, inducing increased central TRP levels, followed in turn by increased central 5-HT synthesis and degradation. In detail, the alterations in peripheral TRP metabolism are related to lower plasma albumin levels and increased plasma free fatty acid concentrations, together resulting in a diminished binding of TRP. These peripheral changes thus induce elevated plasma free TRP concentrations, which are presumably an important factor in the determination of brain TRP concentration. As the peripheral TRP binding derangements have been worked out fairly substantially, as far as concerning this hypothesis (Krause, 1979 b. 1980), we will engage mainly in the investigation in more detail of changes in central 5-HT metabolism.

Based on their design two groups of experiments can be discerned: in part 11 experiments investigating correlations between cancer anorexia and central serotonin activity will be discussed, while in part III results of different interventions in the central serotonin system will be reviewed.

\subsection{Outline of the experimental work}

2.2.1. Experiments, designed to investigate correlations between altered brain serotonin metabolism and cancer anorexila (part II)

Considering increased central serotonin activity as a causative factor in the development of cancer anorexia requires, in experiments seeking to demonstrate the presence of correlations, a group that is matched in food intake with the tumor bearing group, to exclude malnutrition as the cause of biochemical changes. Thus, in all experiments discussed in this section, a pair-fed (PF) control group is included in addition to the TB group and the non TB controll (FF) group, that have free access to food. This PF group consists of animals paired in weight to TB animals at the beginning of each experiment, that had free access to water as the other groups, but were presented with the amount of chow, eaten by their TB counterparts during the previous 24 hour period.

In addition to replicating the original findings by Krause, and further defining this tumor model, which demonstrates limited cachexia, it was important to investigate whether 5-HT activity was also increased in a more chronic. stable model of anorexia and severe cachexia. Another argument supporting a role for serotonin in cancer anorexia could be altered CNS 5-HT activity preceding changes in the behavioral parameter under investigation. Only in the presence of a predictable onset of anorexia in the course of tumor growth, could such a study be performed. Therefore this study was conducted in the acute, but highly reproducible $W_{256}$ tumor model. Finally an effort was made to investigate which brain areas exhibit the increased CNS S-HT 
activity in TB animals. This study was carried out in both the acute and chronic tumor models.

\subsubsection{Efforts to manipulate the entry of tryptophan into the brain and the indoleamine system in cancer anorexia (part III)}

Manipulating the TRP - 5-HT system in tumor bearing animals requires a drug treated control group to exclude drug effects on food intake through mechanisms other than the anorexia causing mechanism currently under investigation. Thus, experiments in this part consist of a TB-group, a FF group, a drug treated TB-group and a drug treated FF group. These experiments were aimed att intervention in processes that determine brain TRP levels or inhibit 5-HT synthesis. Furthermore, experiments that employ drugs known to antagonize 5-HT postsynaptic activity or to intervene chemically in 5-HT synthesis, were conducted. Finally effects of direct chemical stimulation of central hunger mechanisms were studied. Because repeated intervention in the indoleamine system, necessary for determination of dose-response relationships of centrally administered drugs, stereotaxically implanted chronic intracranial cannulae were required. To fulfill this requirement a larger animal model was needed in order to have sufficient skull surface and strength to holld these cannulae. Furthermore, a model displaying a more prolonged anorexia was required for these studies. Prior to our use of the methylcholanthrene induced (MCA) sarcoma model, we conducted several experiments to establish the proper dose and route of inoculation (im. or Sc.) of $W_{256}$ tumor cells in adult female SD rats to obtain a stable, reproducible model of prolonged anorexia. As has been observed previously with the $W_{256}$ tumorline (progress report to the N.C.I. on Studies on the Biology of the Walker 256 carcino sarcoma, sept. 1966,

Arthur D. Little Inc.) we obtained only a $80 \%$ take of the tumor in adult SD rats, and observed even then spontaneous tumor regression. Although the animals became anorectic for some time, substantial numbers of these rats overcame the anorexia and the tumor.

The MCA tumor model presented us with a model of stable prolonged anorexia in adult rats (Popp, 1980). Therefore, we performed all studies requiring repeated intracerebral drug administration, in this MCA sarcoma model.

\subsection{Methodological considerations}

\subsubsection{Animals}

Tumor bearing animals and non tumor bearing freely feeding control rats had ad libitum access to standard laboratory rat chow (Ralston Purina Co., St. Louis, MO.) or test diets, if specified, and water.

All rats were individually housed under a $12 \mathrm{hr}$ light-dark cycle in a temperature and humidity contralled environment.

Daily body weights, food intake and, in specified experiments, water intake were recorded to the nearest tenth of a gram, with appropriate correction being made for food spillage and body weight difference.

\subsubsection{Tumor models}

\section{A. Walker 256 carcino sarcoma}

Walker 256 carcino sarcomas, originally obtained from EG and G Mason Research Institute, Worcester, MA, were maintained in stock by harvesting ascites fluid 5 days after the ip. injection in female 60-80 g Sprague Dawley (SD) rats (Gibco Animal Resources Laboratories, Madison, Wisconsin) of $1 \times 10^{6}$ viable tumor cells. In the 
experiments $W_{256}$ tumors were induced in the hind limb muscle of $60-809$ female SD-rats by injection of a tumor cell suspension containing $5 \times 10^{4}$ viable $W_{25 \%}$ tumor cells in $0.1 \mathrm{ml}$. The viability was assessed after dilution of the ascites fluid 1: 10 with Trypan blue $0.2 \%$. by counting the uncoloured tumor cells in a hemocytometer. Control animals received a sham injection of normal saline at the same site.

B. Methylcholanthrene induced sarcoma.

Mlethylcholanthrene induced sarcomas were originally obtained from M. Brennan MD., N.C.I., Bethesda MD., and maintained in stock by sc. injection in adull (225-250 g) male Fisher 344 rats (Charles River Laboratories, Wilmington, MA.) of a cell suspension containing $2 \times 10^{6}$ viable tumor cells in $0.1 \mathrm{ml}$. The tumor cell suspension was obtained by trypsinizing viable tumor tissue. Tumor cells in this suspension were counted after 1 : 10 dilution with Trypan blue $0.2 \%$ in a hemocytometer, while viability was assessed by Trypan blue exclusion.

In the experiments, MCA tumors were induced in TB animals as described above, while controls received an equal volume of normal saline.

\subsubsection{Biochemical assays}

\section{Plasma}

After sacrifice by decapitation blood was collected from the cerwical wound in heparinized beakers and immediately transferred into glass stoppered tubes. After centrifugation at $4^{\circ} \mathrm{C}$ plasma was frozen and stored at $-70^{\circ} \mathrm{C}$ until assay.

\section{A. Tryptophan}

Plasma free tryptophan was determined in $50 \mu \mathrm{l}$ of an ultrafiltrate prepared by centrifugation of $1.0 \mathrm{ml}$ plasma ( $\mathrm{pH}$ adjusted to $7.4 \pm 0.05$ with $2.5 \mathrm{~N} \mathrm{HCl}$, Corning pH meter 130, Medford, MA.) $\left(100 \times \mathrm{g}, 25 \mathrm{min.} 20^{\circ} \mathrm{C}\right)$ in a CF 50 Diaflo membrane cone (Amicon Corp., Lexington, MA.). Free and total TRP were assayed fluorometrically by the method of Dencla and Dewey (1966) as revised by Bloxam and Warren (1974) and read on an Aminco Bowman fluorometer. Values are reported as $\mathrm{nmol} / \mathrm{m} / \mathrm{l}$ of plasma.

B. Albumin:

Albumin was measured using the bromcresol green method as described by Doumas (1971). Results are reported as $\mathrm{g} / \mathrm{dl}$ of plasma.

C. Amino Acid Analysis:

For amino acid determination $0.5 \mathrm{ml}$ of plasma was deproteinized by addition of $1.5 \mathrm{ml}$ of a solution of $5 \%$ sulfosalicylic acid previously adjusted to $\mathrm{pH} 1.8$ with saturated Li-OH. After protein precipitation samples were centrifuged $\left(10.000 \times 9,25 \mathrm{~min} .4^{\circ} \mathrm{C}\right)$ and the supernatants were passed through a millipore filter $(0.45 \mu \mathrm{m}$ pore sizel before storage at $-70^{\circ} \mathrm{C}$ until analysis. Amino acids were determined on $50 \mu$ of each sample by a Beckman $121 \mathrm{MB}$ automated amino acid analyzer. Amino acid concentrations are reported as $\mathrm{nmol} / \mathrm{ml}$ of plasma.

\section{Brains}

Brains were removed immediately after decapitation, dissected, frozen on dry ice or in liquid nitrogen and stored at $-70^{\circ} \mathrm{C}$ until determination. Brain 5-HT and 5-HIAA were determined fluorometrically following acid-butanol extraction, according to the method of Curzon and Green (1970), with 5-HT and 5-HIAA being complexed with O-phthalaldehyde (OPT) (Sigma Chem. Co., St. Louis, MO.), and read on the Aminco Bowman fluorometer. Brain TRP was extracted in the same fraction as 5-HT and assayed fluorometrically according to the procedures utilized for plasma TRP. TRP values are reported as $\mu \mathrm{g} / \mathrm{g}$ wet brain tissue, while results of 5-HT and 5-HIAA determinations are given as $\mathrm{ng} / \mathrm{g}$ wet tissue.

Brain norepinephrine (NE) and dopamine (DA) were assayed using high performance liquid 
chromatography (HPLC) with electrochemicall detection (Maruyama, 1980). Brain hemisections were homogenized in 5 volumes of $0.4 \mathrm{M} \mathrm{HClO}_{4}$ solution containing (per $100 \mathrm{mi}$ ) $50 \mathrm{mg}$ EDTA, $100 \mathrm{mg} \mathrm{Na} \mathrm{S}_{2} \mathrm{O}_{5}$ and $10 \mu \mathrm{g}$ of alpha methyl $\mathrm{NE}$ (as an internal standard). After centrifugation $\left(31.000 \times 9.15 \mathrm{~min} .4^{\circ} \mathrm{C}\right)$, shaking $(10-15 \mathrm{~min})$ with alumina (100 mg) and washing (3 times with $10 \mathrm{ml} \mathrm{H} \mathrm{H}_{2} \mathrm{O}$ ), each sample was eluted with $1.0 \mathrm{ml}$ of $0.1 \mathrm{~N} \mathrm{HCl}$ solution (containing 10-5 $\mathrm{M} \mathrm{Na}_{2} \mathrm{~S}_{2} \mathrm{O}_{5}$ ) and centrifuged (1000 $\times \mathrm{g} .10 \mathrm{~min}$.). Supernatants $(20 \mu$ aliquots) were injected into the HPLC system (Beckman model $110 \mathrm{~A}$ pump, Altex Reverse phasie $\mathrm{C}_{18}$ column, Palo Alto, CA; Bioanalytical Systems Inc. Model LC-14 electrochemical detector, $W$. Lafayette, IN) with NE eluting $4.5 \mathrm{~min}$. and DA $9.0 \mathrm{~min}$. after the injection. Values are reported as $n g / g$ brain tissue.

\subsubsection{Statistics}

Results obtained are given as the mean \pm standard error of the mean. The data from experiments employing two groups were statistically evaluated using Student's t-test for unpaired observations. If 3 or more groups were to be included, data were evaluated using the Analysis of Variance followed, if needed, by Scheffe's t-test for significance. When correlation coefficients were calculated, significance was tested using the formula:

$t=r \sqrt{\frac{n-2}{1-r^{2}}}$, whereby the $t-t a b l e$ was entered at $n-2$ degrees of freedom. 


\section{Part 11:}

Experiments, designed to investigate correlations between altered brain serotonin metabolism and cancer anorexia 


\section{Chapter 3}

\section{Replication of tryptophan and serotonin system changes in and further definition of the Walker 256 carcino sarcoma model of cancer anorexia}

\subsection{Introduction}

In addition to the confirmation of biochemical changes in tumor bearing (TB) animals regarding plasma amino acid patterns, as well as central nervous system (CNS) indoleamine changes, as observed by Krause et al. $\left(1979^{a+b}, 1980\right)$ it seemed important to assess the onset of anorexia as related to the presence and developiment of the tumor.

Furthermore, it was of interest to investigate whether cachexia was present in this particular tumor model, and if so, what temporal relationship the development of cachexia would have to the onset and development of anorexia. If no cachexia could be demonstrated, we would deal with a tumor-host relationship leading to spontaneous death without depletion of protein/energy stores of the host, in itself no unknown observation (Lundholm, 1980).

\subsection{Materials and methods}

\section{Design}

One hundred and fifty four immature (60-80 g) female SD rats served as subjects across four experiments. In experiment 1,8 rats were inoculated $\mathrm{im}$. with $5 \times 10^{4} \mathrm{~W}_{256}$ tumor cells, while 8 pair fed and 8 freely feeding controls received sham injections of normal saline.

Since in pilot studies spontaneous death in this tumor model had occurred in $80 \%$ of the TB animals on day 10 , all animals in experiment 1 were sacrificed on day 9 post tumor implantation.

In experiment 2, rats were again divided into 3 groups: 6 rats were implanted im. with the $W_{255}$ tumor, whille $P F(n=6)$ and $F F(n=6)$ controls received equal volumes normal saline. As in experiment 1, all animals were sacrificed on day 9 post tumor implantation.

Experiment 3 was of similar design, with 6 rats beling assigned to both TB and PF groups, and 4 animals to the FF group. In this experiment all rats were housed in metabollic cages. In addition to the routine dally monitoring for food intake and body weight, 24 hr urine productiom per animal was collected on $3 \mathrm{cc} 12 \mathrm{~N} \mathrm{HCl}$. After recording of volumes an aliquot of each portion was frozen and stored at $-70^{\circ} \mathrm{C}$ for later determination of nitrogen and 3-methylhistidine contents. Again, animals were sacrificed on day 9 .

In experiment 4 , the 3 groups consisted of 32 rats each. Pilot studies and the results of the previous 3 experiments demonstrated significant anorexia invariably present on day 6 post tumor implantation. Therefore equal numbers of rats from each group were sacrificed on days $3,5,7$ and 9 after the induction of tumor.

In experiments 1 and 4 upper hind limb circumference of the tumor bearing leg and contralaterall leg were measured as an index for tumor presence and tumor girowth.

\section{Dissection}

Experiment 1: blood and brains were taken at sacrifice for determination of levels of plasma. total and free TRP, albumin, amino acids and brain TRP, 5-HT and 5-HIAA. Tumors were carefully dissected, weighed and their volumes measured. 
Experiment 2: blood and brains were collected for determination of brain uptake indices according to the technique described below.

Experiment 3: al sacrifice blood was collected for determination of glucose, hemoglobine. hematocrit, red and white blood cell counts and finally total body water using the tritiated water isotope dilution technique.

Experiment 4: blood was collected from all animals sacrificed in this experiment for plasma amino acid analysis. Livers were removed and quadriceps muscles of the non-tumor bearing limbs were carefully dissected for determination of wet and dry weights and soluble protein contents.

\section{Blood-brain transport of TAP}

Studies of the blood-brain transport of TRP in experiment 2 were performed according to the technique of Oldendorf (1971). Rats were anesthetized with sodium pentobarbital (Pentobarbital) $35 \mathrm{mg} / \mathrm{kg} \mathrm{B.W}$. intraperitoneally. A midline neck incision was made to expose the left common carotid artery, which was cannulated with a 27 gauge needle. A rapid injection of $0.2 \mathrm{ml}$ of a mixture containing $1.0 \mu \mathrm{Ci}$ of ${ }^{3} \mathrm{H}_{2} \mathrm{O}$ and $0.2 \mu \mathrm{Ci}$ of ${ }^{14} \mathrm{C}$-labeled TFP in Krebs-Ringer buffer aidjusted to $\mathrm{pH} 7.4$ with $10 \mathrm{mM}$. Hepes, was made without occluding blood flow. Exactly 15 seconds after the injection, the rat was decapitated and the brains were immediately removed.

A sample of brain tissue ipsilateral to the injection and rostral to the midbrain was extruded through a 20 gauge syringe needle into $1.0 \mathrm{ml}$ NCS tissue solubilizer (American/Searle. Arlington Heights, 1II.)

The brain tissue samples and the injection solutions were analyzed by double-isotope liquid scintillation counting (Packard Tri-Carb). The brain content of the rapidly transported amino acid was compared to the uplake of the freely-diffusable reference, ${ }^{3} \mathrm{H}_{2} \mathrm{O}$. Brain uptake index (BUI) was calculated as:

$$
\frac{\text { (DPM } \left.^{14} \mathrm{C} / \mathrm{DPM}^{3} \mathrm{H}\right) \text { brain }}{\left(\mathrm{DPM}^{14} \mathrm{C} / \mathrm{DPM}^{3} \mathrm{H}\right) \text { injectate }} \times 100
$$

All radioactive substances were obtained from New England Nuclear Corporation (Boston, MA).

\section{Biochemical analysis}

Plasma amino acids, total and free TRP, albumin and brain TRP, 5-HT and 5-HIAA were determined according to standard techniques (Chapter 2). Glucose was assayed using a kinetic glucose oxidase method on the JL 919 at $37^{\circ} \mathrm{C}$ (Instrumentation Lab., Inc.), while hematological values were obtained from a Coulters' coulter counter.

Urine total nitrogen was analyzed, using the Kjeldahl method, while 3-methylhistidine content was analyzed after hydrolyzing (Young, 1972) on the Beckman 121 MB automated amino acid analyzer.

Dissected tissues were weighed on a Mettler balance (P 163), and muscles and livers were lyophyllized in a Virtis lyophyllizer (TO 82) for water content determinations. Parts of these tissues were after weighing homogenized in $20 \mathrm{ml} 0.01 \mathrm{M}$ TRIS buffer. After centrifugation (100 $\times$ g, $25 \mathrm{~min}, 20^{\circ} \mathrm{L}$ ) total soluble protein was determined using a Bio Rad Protein Assay Kit (Bio Rad Laboratories).

\subsection{Results}

\section{Experiment 1}

Intramuscularly implanted Walker 256 carcino sarcoma cells induced in immature female SD 


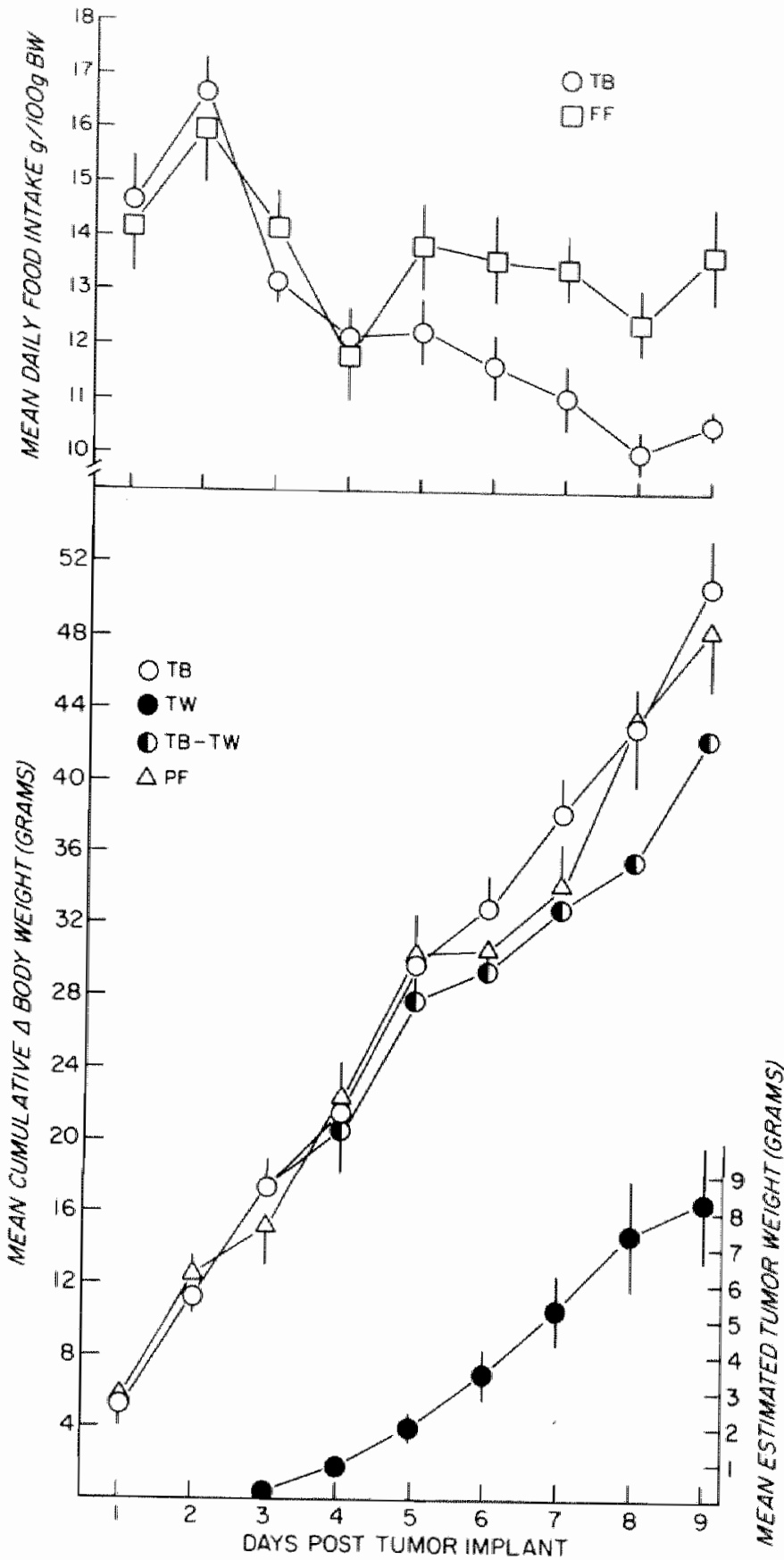

Fig. 3.3.-1. Mean ( $\mathrm{SEM}$ ) daily food intake of tumor bearing (TB) and freelly feeding (FF) control rats, mean (I SEM) change in body weight of TB and pair-fed control (PF) rats and mean (ISEM) calculated tumor weight (TW). On day O TB rats were injected (im.) with $5 \times 10^{4}$ viable $W_{2 s i}$ tumor cells. while PF and $F F$ rats received a sham injection normal saline. Significant $(p<0.05)$ anorexia was observed from day 6 until sacrifice on day 9 . Body weight on day 0 was reference weight. Each data point reflects $n=8$. 


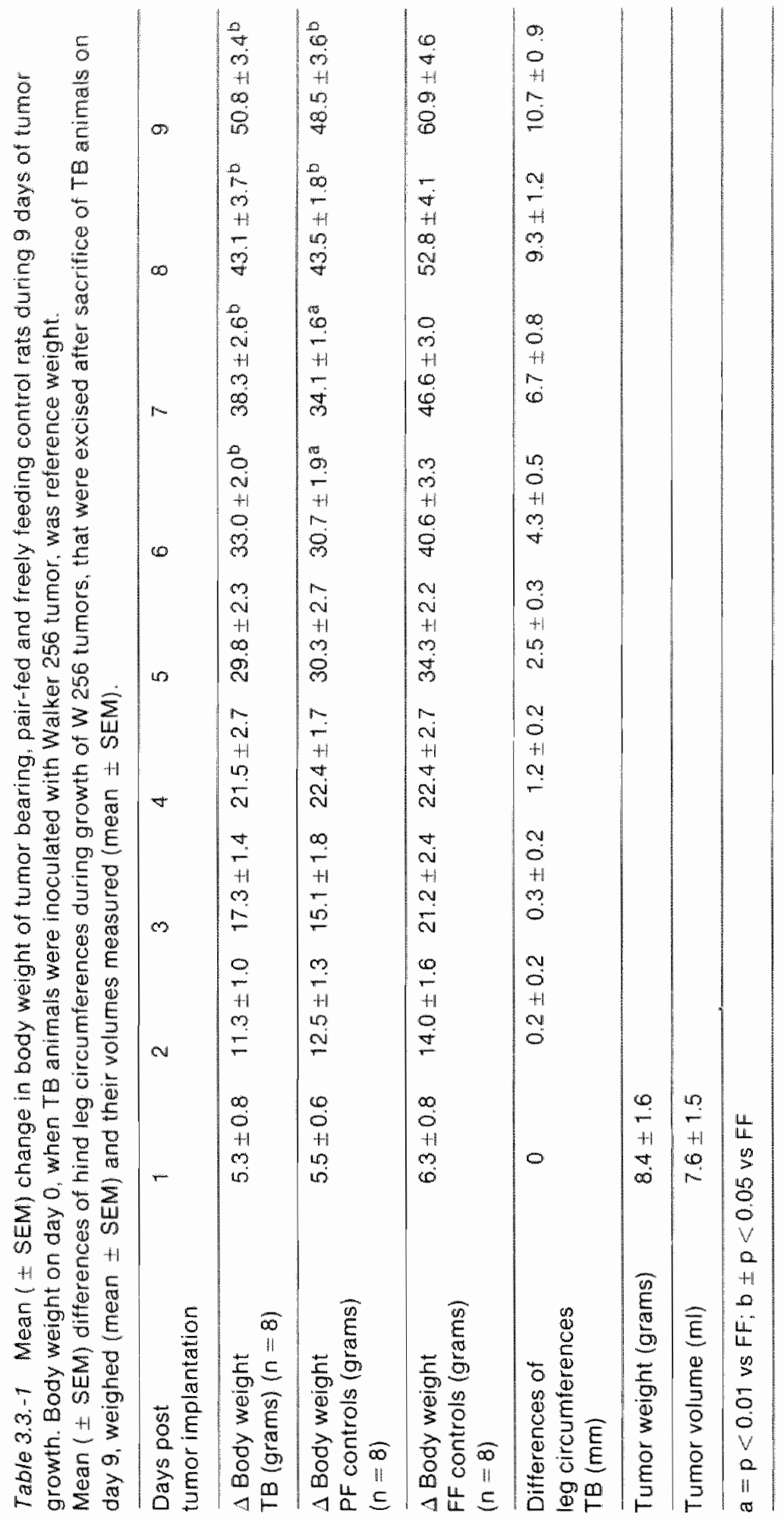


rats a significant $(p<0.05)$ reduction of food intake from day 6 , that increased in severity as the tumor grew (Fig. 3.3.-1).

Mean changes of body weight did not differ across days between TB and PF groups, even when body weights of TB rats were corrected for tumor weight (Fig. 3.3. - ). Both TB and PF rats however gained significantly $(p<0.05)$ less weight from day 6 , than did FF animals (Table 3.3.-1). This observation suggests, that diminished growth in TB animals in this tumor model is a result of diminished food intake, with a small tumor $(8.4 \pm 1.6 \mathrm{~g})$ not inducing significant additional weight loss in TB animals. These results confirmed our clinical impression that this tumor model exhibited very limited cachexia.

Tumors were palpable from day 5 . From day 4 tumor bearing leg function was becoming increasingly impaired, although this impairment did not seem to limit the animals in their moving.

Callculation of leg circumference differences between the tumor and non-tumor bearing hind legs, resulted in appreciation of tumor presence on day 3 . This technique assumes that normal muscle tissuie on the ipsilateral leg is not displaced or invaded by tumor tissue.

Dissection of tumors on day 9 indicated however that hardly any normal tissue remained at the site of the tumor (Table 3.3.-1). Though unorthodox, calculation of weight per change of tumor leg circumference on day 9 , provided an opportunity to obtain a tumor growth curve, thus giving an impression of tumor growth as it related to the onset of anorexia and weight loss (Fig. 3.3.-1).

Evaluation of results of biochemical analyses using a one-way ANOVA revealed significant differences between groups for albumin, total and free TRP, brain TRP, serotonin, and 5-HIAA (Table 3.3.-2). Post hoc t-tests indicated significantly lower plasma albumin levels in $\mathrm{TB}$ animals as compared to both control groups. Plasma free TRP was increased in TB animals, although the total concentration of this AA was decreased. This latter finding seemed diet induced, since it was also present in PF animals. Furthermore, brain TRP levels were elevated in TB animals, as were brain levels of serotonin and 5-HIAA in this group, as compared to both control groups.

\section{Experiment 2}

Since considerable evidence was being collected in this laboratory suggesting that in rats, blood-brain barrier transport of neutral amino acids is increased after porto-caval

Table 3.3.-2 Mean ( \pm SEM) levels of plasma albumin, total tryptophan (TRP), free TRP, and brain TAP, serotonin (5-HT) and 5-hydroxyindoleacetic acid (5-HIAA) in tumor bearing (TB), pair-fed (PF) and freely feeding (FF) control rats 9 days after induction of Walker 256 tumors. Between parenteses $F$ and $p$ values of analysis of variance of each factor, at the bottom p values of post hoc t-tests.

\begin{tabular}{|c|c|c|c|c|}
\hline Plasma & $\begin{array}{l}\text { TB } \\
n=8\end{array}$ & $\begin{array}{l}P F \\
n=8\end{array}$ & $\begin{array}{l}\mathrm{FF} \\
n=8\end{array}$ & \\
\hline $\begin{array}{l}\text { Albumin }(\mathrm{g} / \mathrm{dl}) \\
\text { Tot. TRP }(\mathrm{mmol} / \mathrm{ml}) \\
\text { Free TRP }(\mathrm{nmol} / \mathrm{ml}) \\
\text { Ratio free TRP/ }\end{array}$ & $\begin{aligned} 1.83 & \pm 0.06 \\
47.1 & \pm 2.9 \\
23.1 & \pm 1.4 \\
0.048 & \pm 0.003\end{aligned}$ & $\begin{array}{c}2.85 \pm 0.18^{a} \\
60.0 \pm 5.6 \\
7.2 \pm 2.1^{a} \\
0.023 \pm 0.002^{a}\end{array}$ & $\begin{array}{c}2.95 \pm 0.05^{a} \\
86.8 \pm 9.6^{b c} \\
10.9 \pm 1.0^{a} \\
0.023 \pm 0.002^{a}\end{array}$ & $\begin{array}{l}(F(2,21)=25.86 ; p<0.001) \\
(F(2,21)=10.80 ; p<0.01) \\
(F(2,21)=31.91 ; p<0.001 \\
(F(2,21)=28.49 ; p<0.001\end{array}$ \\
\hline \multicolumn{5}{|l|}{ Brain } \\
\hline $\begin{array}{l}\text { TRP }(\mu \mathrm{g} / \mathrm{g}) \\
5-\mathrm{HT}(\mathrm{ng} / \mathrm{g}) \\
5 \text {-HIAA }(\mathrm{ng} / \mathrm{g})\end{array}$ & $\begin{array}{r}4.79 \pm 0.25 \\
822.4 \pm 20.8 \\
733.8 \pm 16.5\end{array}$ & $\begin{array}{l}3.47 \pm 0.17^{b} \\
720.3 \pm 17.7^{b} \\
548.0 \pm 8.1^{a}\end{array}$ & $\begin{array}{l}3.80 \pm 0.21^{b} \\
713.6 \pm 9.1^{a} \\
543.8 \pm 14.5^{a}\end{array}$ & $\begin{array}{l}(F(2,21)=10.46 ; p<0.01) \\
(F(2,21)=13.44 ; p<0.001) \\
(F(2,21)=64.77 ; p<0.001)\end{array}$ \\
\hline
\end{tabular}

$a=p<0.001$ vs TB; $b=p<0.01$ vs TB; $c=p<0.05$ vs PF 
Table 3.3.-3 Mean ( SEM) brain uptake indices (BUI) for tryptophan, oblained through Oldendori's technique, 9 days after im. induction of $W_{256}$ tumors in tumar-bearing (TB) rats. or sham injection normal saline in pair-fed (PF) and freely feeding (FF) control rats.

Between parenteses results of a one-way analysis of wariance.

\begin{tabular}{lllll} 
& $\begin{array}{l}\text { TB } \\
n=6\end{array}$ & $\begin{array}{l}\text { PF } \\
n=6\end{array}$ & $\begin{array}{l}\text { FF } \\
n=6\end{array}$ & \\
\hline BUi $(\%)$ & $47.2 \pm 5.8$ & $47.6 \pm 4.9$ & $41.5 \pm 3.5$ & $(F(2.15)=0.54 ;$ N.S.) \\
\hline
\end{tabular}

anastomosis, as well as in uremia and sepsis, we investigated this phenomenon in TB rats. In addition, since serotonin was significantly elevated in TB animals, in contrast to Krause's earlier reports, the need to exclude increased BBB transport activity as a source of these changes was apparent. Behavioral parameters in this experiment replicated those of the first experiment. Thus, food intake by TB animals was significantly decreased on day 6 post tumor implantation. Brain uptake indices for TRP determined by Oldendorfs' technique, revealed no indication for increased BBB activity in TB rats (Table 3.3.-3). These resullts therefore replicate Krause's data.

\section{Sublective observations}

Tumor bearing animals in both experiments displayed altered activity. Thus, while PF and FF would sleep during the light period, TB animals would stay awake, and appeared to eat more frequently during this period. As the tumor grew, the animals became increasingly lethargic, their breathing heavier and apparently more difficult. Their ears and eyes became very pale.

On handling the lack of emaciation in the tumor host was most striking, thus confirming the data from experiment 1.

Necropsy showed metastasis being limited to the regional, inguinal lymph nodes.

No intraperitoneal, liver, spleen, lung or brain metastasis could be demonstrated. Tumors exhibited to a limited degree central necrosis, and appeared clinically fairly well encapsulated.

Table 3.3.-4 Mean ( \pm SEM) values of glucose, hemoglobin, hematocrit, red blood cell count (RBC), white blood cell count (WBC), and total body water, obtained at sacrifice 9 days after tumor induction in TB animals.

Between paranteses results of analysis of variance of each item, at the bottom $p$ values of post hoc 1-tests.

\begin{tabular}{|c|c|c|c|c|}
\hline & $\begin{array}{l}\text { TB } \\
n=6\end{array}$ & $\begin{array}{l}\mathrm{PF} \\
n=6\end{array}$ & $\begin{array}{l}F F \\
n=4\end{array}$ & \\
\hline Glucose (mg/dil) & $62.3 \pm 22.5$ & $106.5 \pm 1.3$ & $109.5 \pm 11.5$ & $(F(2,13)=3.21 ; N . S)$. \\
\hline Hemoglobin (mg/dl) & $11.1 \pm 0.6$ & $12.6 \pm 0.3^{b}$ & $12.3 \pm 0.2$ & $(\mathbb{F}(2,13)=5.01 ; p<0.05)$ \\
\hline Hematocrit $(\%)$ & $32.4 \pm 1.1$ & $34.3 \pm 0.7$ & $33.1 \pm 1.0$ & $(F(2,13)=1.29 ;$ N.S. $)$ \\
\hline $\operatorname{RBC}\left(\times 10^{12 / 1}\right)$ & $5.2 \pm 0.2$ & $5.5 \pm 0.2$ & $5.4 \pm 0.03$ & $(F(2,13)=1.24 ;$ N.S. $)$ \\
\hline WBC $(\times 109 / 1)$ & $17.9 \pm 3.3$ & $7.6 \pm 0.6^{\mathrm{a}}$ & $9.0 \pm 0.5^{b}$ & $(F(2,13)=10.14 ; P<0.01)$ \\
\hline Total body water & $109.2 \pm 1.8$ & $119.2+6.3$ & $118.5 \pm 6.8$ & $(F(2,13)=1.05 ; N . S)$. \\
\hline
\end{tabular}


Finaliy, a most intriging obsenation was that TB animals would die without obvious signs of disseminated cancerous disease.

\section{Experiment 3}

In an effort to identify the onset of catabolic processes as they relate to the development of anorexia, daily nitrogen balances and urinary 3-methylhisticine excretion were determined in this experiment. Thus, nitrogen balances would reflect total body nitrogen flux, and also represent tumor metabolism. 3-Methylhistidine, being a degradation product of actin and myosin, that is not reutilized for protein synthesis (Young, 1972), would reflect muscie breakdown (Munro, 1977), since about $84 \%$ of the total 3-methylhistidine excreted is derived from skeletal muscle tissue (Nishizawa, 1977).

The results of nitrogen-balance determinations revealed no statistically significantly differences among groups on any day (Fig. 3.3.-2). However, some indication of a decreased positive $\mathrm{N}$-balance on day 9 existed in both TB and PF groups, suggesting dietary intake as the cause for this phenomenon. In addition, the positive $\mathrm{N}$-balances undoubtedly reflect immaturity of the experimental animals, resulting in $N$-retention for growth.

Tumor grawth, and thus tumor $\mathrm{N}$-retention, did not induce a significantly different $\mathrm{N}$-balance in TB-animals. This observation, however, does not necessarily mean that no shifts in $\mathrm{N}$-flux between host and tumor took place.

3-Methylhistidine excretion did not differ between groups on any day (Fig. 3.3.-3). Fair-fed animals maintained the same level of 3 -methylhistidine excretion during the experiment suggesting the starwation effect in this tumor model being a very mild one. Thus, nitrogen sparing mechanisms were not activated, which are presumably associated with decreased 3-methylhistidine excretion (Munro, 1977). The lack of elevation of 3-methylhistidine excretion in TB animals as compared to both control groups, in combination with the earlier mentioned $\mathrm{N}$-balance results, suggests no increased $\mathrm{N}$-release from muscle in $\mathrm{TB}$ animals.

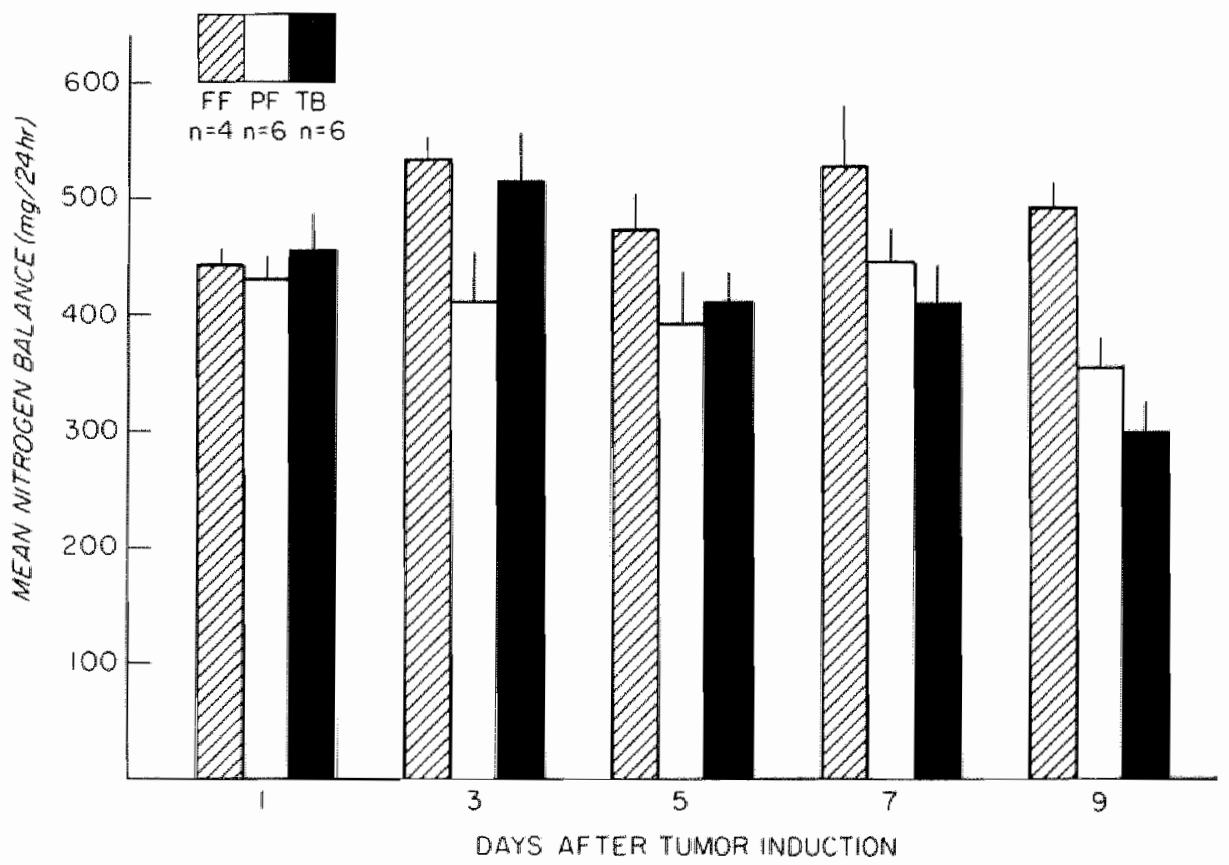

Fig. 3.3.-2 Mean (i. SEM) daily nitrogen balance of freely feeding (FF) and pair-fed (PF) controls. and Walker 256 tumor bearing (TB) rats. 


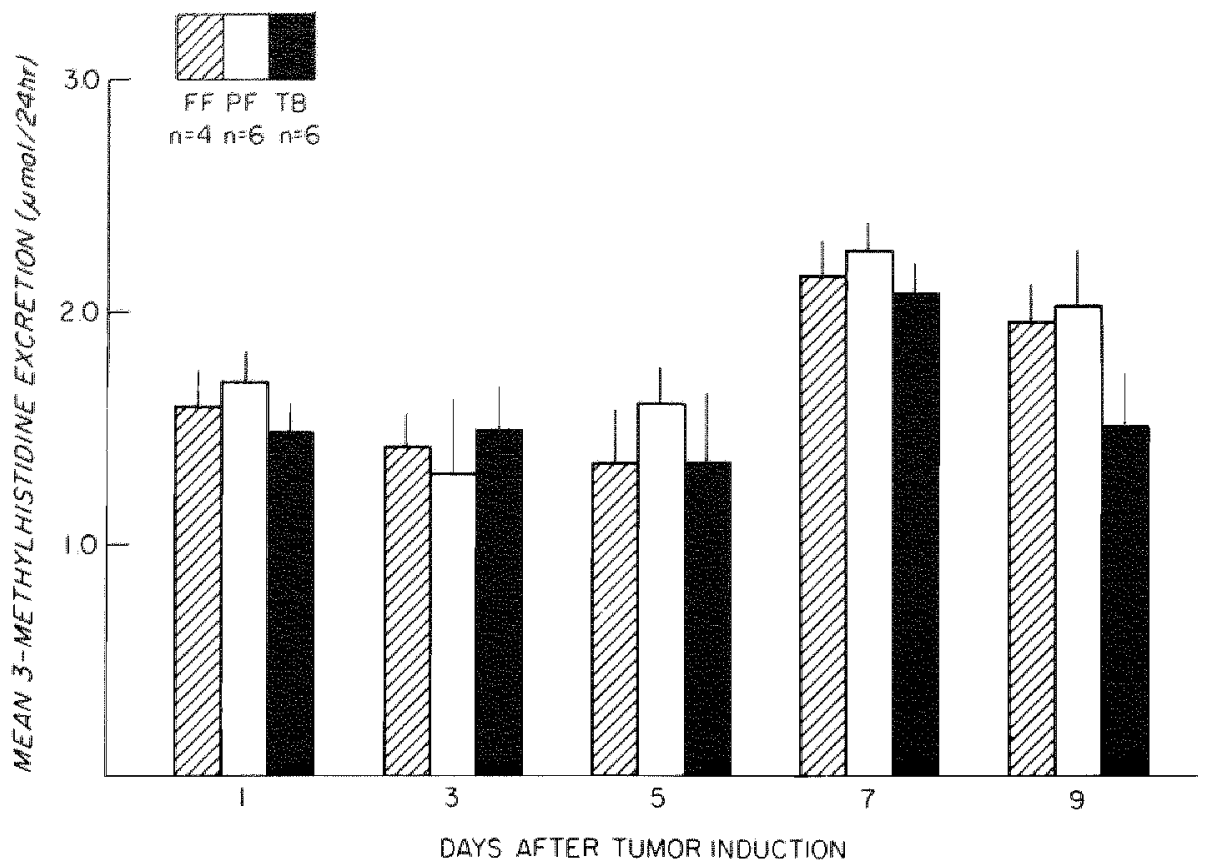

Fig. 3.3.-3 Mean (t: SEM) daily urinary emcretion of 3-methylhistidine by freely feeding (FF) and pair-fed (PF) control rats, and Walker 256 tumor bearing (TB) rats.

In addition to the above determinations, parameters shown in table 3.3.-4 were evaluated in an effort to clarify some of the clinical observations made thus far. Thus, one-way ANOVA on results of biochemical determinations of glucose, hematocrit. $\mathrm{RBC}$ and total body-water showed no differences across groups. Furthermore, ANOVA on hemoglobine and WBC values revealed significant changes $(F(2.13)=5.01 ; p<0.05$, and $F(2.13)=10.14: p<0.01$ respectively). Post hoc t-tests however showed a consistent change between TB-animals and both control groups, only for WBC. This leucocytosis possibly reflects tumor necrosis.

\section{Experiment 4}

Analysis of mulscle tissue seemed to confirm the above findings (Table 3.3.-5). Thus, a two-way analysis of variance indicated changes in wet weight $(F(2,84)=17.09 ; p<0.001)$ and dry weight $(F[2.84)=13.35 ; p<0.001)$, with no changes being observed for water content and water soluble protein content of muscle tissue. One-way ANOVA conducted across the 4 test days indicated for all groups significantly increased wet weights (TB: $F(3.28)=73.33 ; p<0.001 ; P F: F(3.28)$

137.09; $p<0.001 ; F F: F(3.28)=32.39, p<0.001)$ and dry weights (TB: $F(3.28)=61.85 ; p<0.001 ; P F: F(3.28)=$ 19.51: $p<0.001 ; \mathrm{FF}: F(3.28)=21.64 ; \mathrm{p}<0.001)$. Daily comparison of the groups revealed significant group differences in wet weight on days $3(F(2,21)=5.63 ; p<0.05), 5(F(2,21=5.55 ; p<0.05)$ and $9(F(2,21)=6.99 ; p<0.01)$, and in dry weight on days $3(F(2,21)=4.13 ; p<0.05)$ and $5(F(2.21)=5.44$; $p<0,051$. Post hoc t-tests showed that FF rats had significantly $(p<0.05)$ increased wet and dry weights as compared to TB and PF groups on days 3 and 5 . In addition wet weights were decreased in the TB group as compared to both control groups on day 9 . Thus, in addition to changes induced by growth, no tumor induced changes could be observed.

Analysis of changes in liver weight and composition (Table 3.3.-6) by a two-way ANOVA indicated significant changes in wet weight $(F(2,84)=25.36 ; p<0.001)$, dry weight $(F(2,84)=9.53$ : $p<0.001)$ and water content $(F(2,84)=7.13, p<0.01)$. One-way ANOVA conducted across the four test days revealed, as in muscle tissue, for all groups significantly increased wet weights 
Table 3.3.-5 Mean ( \pm SEM) weights, water content and soluble protein content of quadriceps muscles from tumor bearing (TB), pair-fed (PF) and freely feeding (FF) control rats $3,5,7$ and 9 days after the injection (im.) of $5 \times 10^{4}$ Walker 256 carcino sarcoma cells in TB animals. For each group on each day $n=8$. At the bottom p values of post hoc t-tests.

Days of sacrifice

\begin{tabular}{llllll}
\hline Muscles & & 3 & 5 & 7 & 9 \\
\hline Wet weight & TB & $0.71 \pm 0.014$ & $0.85 \pm 0.016$ & $1.09 \pm 0.022$ & $1.12 \pm 0.032$ \\
(grams) & PF & $0.72 \pm 0.019$ & $0.83 \pm 0.016$ & $1.14 \pm 0.024$ & $1.21 \pm 0.014$ \\
& FF & $0.82 \pm 0.035^{a b}$ & $0.93 \pm 0.023 \mathrm{ab}$ & $1.19 \pm 0.040$ & $1.27 \pm 0.037^{a}$ \\
& & & & & \\
Dry weight & TB & $0.18 \pm 0.003$ & $0.21 \pm 0.005$ & $0.28 \pm 0.008$ & $0.29 \pm 0.009$ \\
(grams) & PF & $0.18 \pm 0.005$ & $0.21 \pm 0.005$ & $0.28 \pm 0.007$ & $0.30 \pm 0.002$ \\
& FF & $0.21 \pm 0.017^{a b}$ & $0.23 \pm 0.006$ ab & $0.29 \pm 0.009$ & $0.31 \pm 0.009$ \\
Water content & TB & $75.3 \pm 0.1$ & $75.5 \pm 0.2$ & $74.8 \pm 0.3$ & $74.6 \pm 0.2$ \\
(\%) & PF & $75.8 \pm 0.2$ & $75.2 \pm 0.2$ & $75.2 \pm 0.3$ & $75.2 \pm 0.2$ \\
& FF & $74.6 \pm 1.0$ & $75.0 \pm 0.2$ & $75.3 \pm 0.1$ & $75.2 \pm 0.4$ \\
Protein & & & & & \\
(mg/g dry & TB & $385.8 \pm 25.9$ & $288.4 \pm 16.5$ & $304.6 \pm 12.3$ & $334.4 \pm 9.6$ \\
weight) & FF & $386.9 \pm 17.7$ & $325.8 \pm 25.3$ & $359.5 \pm 14.9$ & $321.8 \pm 12.3$ \\
\hline
\end{tabular}

$a=p<0.05$ vs TB; $b=p<0.05$ vs PF

Table 3.3.-6 Mean ( \pm SEM) weights, water content and soluble protein content of livers from tumor bearing (TB), pair-fed (PF) and freely feeding (FF) control rats $3,5,7$ and 9 days after the injection (im.) of $5 \times 10^{4}$ Walker 256 carcino sarcoma cells in TB amimals.

For each group on each day $n=8$. At the bottom $p$ values of post hoc $t$-tesits.

Days of sacrifice

\begin{tabular}{llllll}
\hline Livers & & 3 & 5 & 7 & 9 \\
\hline $\begin{array}{l}\text { Wet weight } \\
\text { (grams) }\end{array}$ & TB & $4.76 \pm 0.088$ & $5.30 \pm 0.064$ & $6.51 \pm 0.153$ & $7.65 \pm 0.228$ \\
& PF & $4.35 \pm 0.074^{\mathrm{a}}$ & $4.65 \pm 0.083^{\mathrm{a}}$ & $5.68 \pm 0.195^{\mathrm{a}}$ & $6.26 \pm 0.165^{\mathrm{a}}$ \\
& FF & $4.41 \pm 0.143^{\mathrm{a}}$ & $5.00 \pm 0.144$ & $6.25 \pm 0.259$ & $7.02 \pm 0.222^{2}$ \\
Dry weight & TB & $1.42 \pm 0.082$ & $1.54 \pm 0.020$ & $1.83 \pm 0.041$ & $2.11 \pm 0.063$ \\
(grams) & PF & $1.32 \pm 0.038$ & $1.37 \pm 0.021^{\mathrm{a}}$ & $1.69 \pm 0.055$ & $1.90 \pm 0.043^{\mathrm{a}}$ \\
& FF & $1.30 \pm 0.048$ & $1.51 \pm 0.044^{\mathrm{b}}$ & $1.88 \pm 0.075$ & $2.13 \pm 0.074^{\mathrm{b}}$ \\
& & & & & \\
Water content & TB & $70.1 \pm 1.7$ & $71.1 \pm 0.2$ & $72.0 \pm 0.2$ & $72.4 \pm 0.4$ \\
(\%) & PF & $69.8 \pm 0.6$ & $70.5 \pm 0.2^{\mathrm{a}}$ & $70.3 \pm 0.2^{\mathrm{a}}$ & $69.6 \pm 0.2^{\mathrm{a}}$ \\
& FF & $70.7 \pm 0.3$ & $69.7 \pm 0.1^{\mathrm{a}}$ & $70.0 \pm 0.1 \mathrm{a}$ & $69.7 \pm 0.2^{\mathrm{a}}$ \\
Protein & & & & & \\
(mg/g dry & TB & $503.0 \pm 28.4$ & $559.1 \pm 14.1$ & $558.3 \pm 17.0$ & $524.1 \pm 14.2$ \\
weight) & PF & $533.1 \pm 33.9$ & $585.0 \pm 38.7$ & $618.4 \pm 16.1$ & $547.6 \pm 8.9$ \\
\hline
\end{tabular}

$a=p<0.05$ vs TB; $b=p<0.05$ vs PF 
(TE: $F(3.28)=70.47: p<0.001: P F: F(3.28)=34.45: p<0.001 ; F F: F(3,28)=26.41 ; p<0.001)$ and dry weights (TB: $F(3,28)=27.44 ; p<0.004 ; P F: F(3.28)=37.17 ; p<0.001 ; F F: F(3.28)=26.99 ; p<0.001)$. Daily comparison of the groups indicated significant group differences in wet weights on days $3(F(2,2)=7.40$ : $p<0.01\}, 5(F(2.21)=3.33 ; p<0.01), 7(F(2,21=4.22 ; p<0.05)$ and $9(F(2.21)=11.01 ; p<0.001)$ in dry weight on days $5(F(2,21)=0.21 ; p<0.01)$ and $9(F(2,21)=4.15 ; p<0.05)$, and in water content on days $5(F(2.21)=15.87 ; p<0.001), 7(F(2,21)=44.46 ; p<0.001)$ and $9(F(2.21)=23.40 ; p<0.001)$. Post hoc - tests indicated that changes in wet weight were caused by increments in the TB group as compared to both control groups on days 3,5 and 9 , and by elevation in the TB group as compared to PF animals on day 7 . Also, dry weights were lower in PF animals as compared to $T B$ and FF animals on days 5 and 9 . Finally liver water content was increased in $T B$ rats on days 5, 7 and 9. These data suggest that increased wet liver weights in TB animals are partly due to increased water content, and partly because dry weights do not decrease in these animals as occurred in PF animals already on day 5, just prior to the onset of anorexia.

\subsection{Discussion}

Since in pilot studies spontaneous death of a considerable number of TB animals occurred on day 10 post intramuscular implantation of $5 \times 10^{4}$ viable Walker 256 carcino sarcoma cells, all experiments were carried through 9 days. During these days a very stable onset of significantly decreased food intake was observed on day 6, that increased in severity as the tumor grew. No literature other than Krause"s $\left(1979^{\mathrm{atb}}, 1980\right)$ reports on food intake behavior in this tumor model. Grossly similar results were obtained, but we were able to measure food intake more precisely.

Although function of the tumor bearing hind leg was severely impaired, this did not seem to restrict the animals in their mobility. However, it is still possible that the observed lethargy accounts for some part in the decline of food intake, as Morrison (1973) observed "a progressive incapacity to undertake the motor activity of feeding'. Early in tumor growth this decreasing motor activity is presumably compensated by increased feeding efficiency, partly through increased meal frequency (Morrison, 1976 ). We seem to confirm these reports with our clinical observations, that are however not quantified.

Unaltered nitrogen retention in any of the groups compared to FF animals does not necessarily result from similar nitrogen sparing mechanisms in these groups. This is illustrated by decreased albumin values in TB animals presumably a result of translocation of nitrogen to the tumor (Karlberg, 1980). These observations are not new. Mider (1948) published observations in $W_{250}$ carcino sarcoma bearing rats, suggesting a similar nitrogen drain to the tumor, while Fenninger (1954) reviewed studies suggesting a similar mechanism in human cancer. The $\mathrm{N}$-balance studies also suggest that the decline of body weight gains In PF animals occurs at the cost of body water and/or fat compartments. Lundholm (1980) described weight loss in PF animals occurring mostly at the cost of the water compartment, in his experiments with the MCA tumor in adult, weight stable mice. The lack of difference in body weight gains between TB and PF animals in the presence of unaltered total body water content in these animals suggests depletions in the same compartments in TB rats as in PF animals. On the other hand this lack of difference is also indicative of the mildness of cachexia in this tumor model, since it is known from other studies, employing models with longer periods of tumor-host interaction, that body lipid content decreases significantly in TB animals (Lundholm, 1980). The mild cachexia is once more Hllustrated by the analysis of muscle tissue, which did not reveal any tumor related alterations, and by the urinary 3-methylhistidine excretion observations. Thus, while in protein-calorie malnutrition 3-methylhistidine excretion is, after an adaptation period, expected to fall (Munro, 1977), no such observation was made in the PF animals. TB animals would be expected to excrete more than control animals excrete (Norton, 1981), based on the assumption that the 
presence of a tumor prohibits the host from adapting its metabolism to chronic starwaton (Brennan, 1977), and provided that no 3-methylhistidine recycling takes place in the tumor. The death of TB-animals in the presence of only mild emaciation, illustrates once more (Lundholm, 1980) that death in cancerous disease is not occurring when tissue energy and nitrogen stores are exhausted, as has been suggested by Sherman (1950).

Some indication of how the presence of a tumor affects host metabiolism as related to the onset of anorexia may be derived from the analysis of liver tissue. Thus, the TB group showed increased water content as compared to both control groups, and increased dry weights as compared to the PF group from day 5, while anorexia was not present until day 6. The latter finding may have many explanations, one of these being increased protein synthesis (Lundholm, 1979), relative to the starvation suffered by these animals. Although some animals were hypoglycemic, resulting in decreased mean values, it is too speculative to suggest that hypoglycemia is the cause of death induced by the tumor. Obwiously massive bleeding, suggested by the pale appearance of TB animals was not involved in the cause of death. Also, death induced by disseminated cancer, invading wital organ systems, and thus impairing their function, is not very likely with no such tumor expansion being observed.

The changes observed in TRP and 5-HT metabolism replicate Krause's findings. Thus, in a model of very limited starvation for both TB and PF animals. TRP metabolism was altered resulting in an increased plasma free fraction of this amino acid in TB animals. Conversely, the total concentration of plasma TRP was decreased in both TB and PF rats, presumably as a result of declined food intake. The differentation of TRP metabolism between TB and PF animals is probably based on the decreased possibility for this amino acid to bind in plasma to albumin, which was lower in TB animals as compared to control groups. Since we were not able to measure FFA concentrations we must assume that these were elevated, as has been described by Krause (1979a+b) and others (Mider, 1948;

Lundholm, 1980).

Since the free fraction of TRP was increased relative to total TRP concentrations, and decreased albumin levels and unaltered competing NAA concentrations were present in TB animals, it did not seem unreasonable to assume that this factor contributed most to tryptophan transport into the brain in these animals (Yuwiler, 1977). Normal BUI values suggest that free TRP concentrations are the more important determinants of the increased brain TRP levels in TB animals. In agreement with Pardridge's (1976) concept of serotonin synthesis rates being dependent on precursor availability, it seems warranted to explain the observed elevated brain 5-HT and 5-HIAA levels as a consequence of the increased TRP levels. Therefore we confirm the results of Krause et al. and demonstrate elevated brain TRP, 5-HT and 5-HIAA levels in TB animals, suggesting increased serotonin synthesis and degradation in these animals. In connection with the literature that suggests serotonim acting as a satiety signal (Goldman, 1971; Kruk, 1973; Blundell, 1975, 1979; Latham, 1979). these observations may suggest seratonin as a primary mediator of cancer anorexia.

\subsection{Conclusions}

1. A Walker 256 carcino sarcoma implanted intramuscularly in immature female SpragueDawley rats results in a tumor modell in which the tumor will kill the host, will induce anorexia on a constant moment in the course of tumor growth, but will not induce the emaciation so commonly associated with cancer.

2. In this $W_{256}$ carcino sarcoma model the tumor will not metastasize other than to regional lymph nodes.

3. The described $W_{256}$ carcino sarcoma model is so acute that no changes can be observed in nitrogen balances or urinary 3-methylhistidine excretions of PF or TB amimals. 
4. While in liver tissue tumor related atterations can be observed, that may be explained, in part, by lack of adaptation to starvation by TB animals, no such changes can be observed in muscle tissue.

5. Total body water is not increased in $W_{256}$ carcino sarcoma bearing animals.

6. No signs of gross bleeding or consistent hypoglycemia are present in $\mathbf{W}_{256}$ carcino sarcoma bearing rats.

7. Significantly increased WBC in TB animals may very well be explained by the presence of, albeit limited, tumor necrosis.

8. Plasma albumin is lowered inducing together with presumed increased levels of FFA, increased plasma free TRP lewels, which may in the presence of normal BBB NAA transport activity be responsible for increased brain TRP levels, that in turn presumably induce increased brain 5-HT synthesis and degradation.

9. Merely by association of this observation with the suggestion from literature of serotonergic mediation of satiety it is hypothesized that cancer anorexia may be mediated, at least in part, by serotonin. 


\section{Chapter 4}

\section{Developmental changes in central serotonergic activity during growth of a Walker 256 carcino sarcoma}

\subsection{Introduction}

If biochemical changes in the TRP and indoleamine system were indeed causative in the development of cancer anorexia, one would expect these biochemical derangements to take place just prior to the onset of anorexia. Since in the acute model of cancer anorexia, the $W_{256}$ carcino sarcoma in immature female SD rats, significantly decreased food intake in the TB group invariably occurs on day 6 post tumor implantation, it became possible to correlate changes in biochemical parameters to the development of behavioral changes.

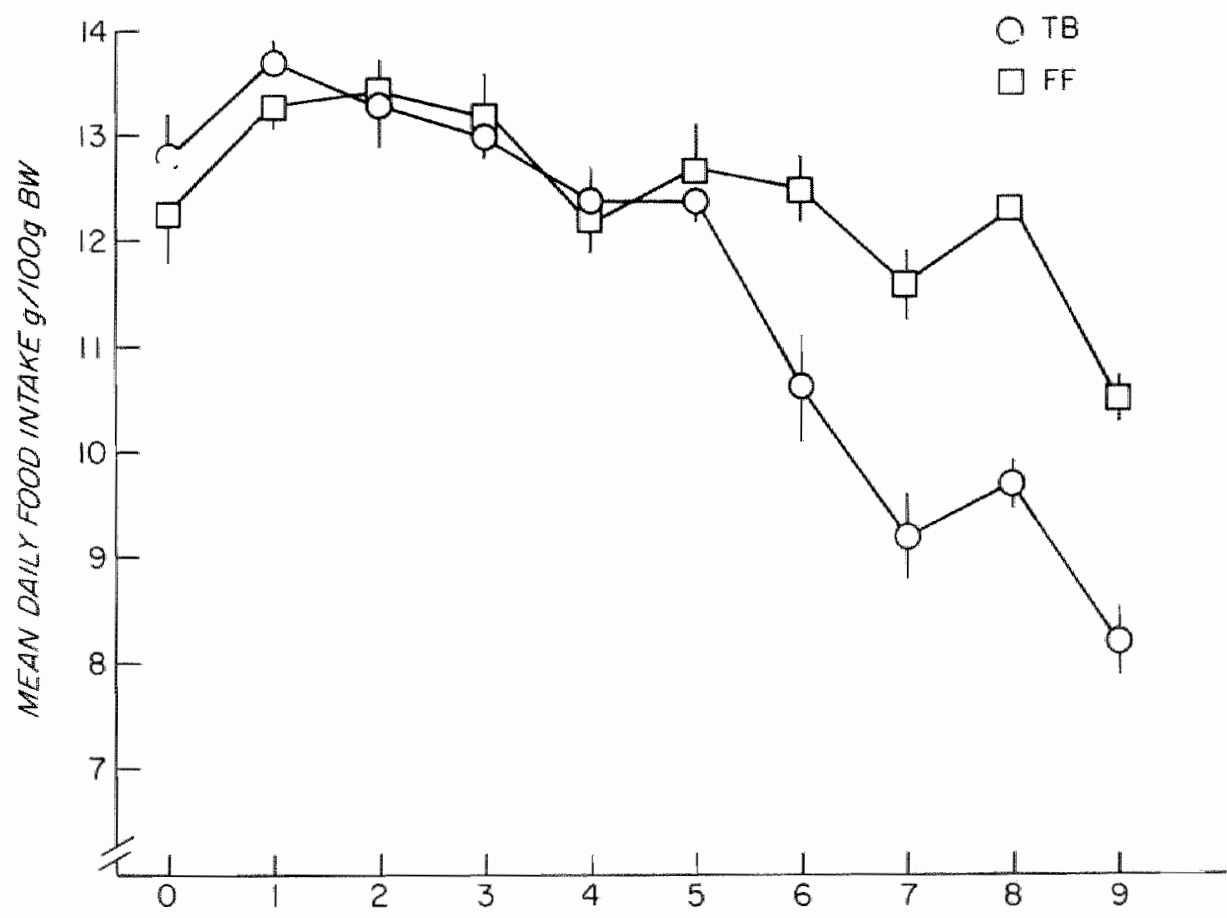

DAYS POST TUMOR IMPLANT

Fig. 4.3.-1 Mean ( 1 SEM) daily food intake of tumor bearing (TB) and freely feeding (FF) control rats. On day $0, T B$ rats were injected $\left(\mathrm{im}\right.$.) with $5 \times 10^{4}$ viable Walker 256 carcino sarcoma cells, while $F F$ rats received normal saline.

Significant $(p<0.01)$ anorexia was observed on day 6 and increased in severity until day 9 . Each dala point reflects $n=8$. 

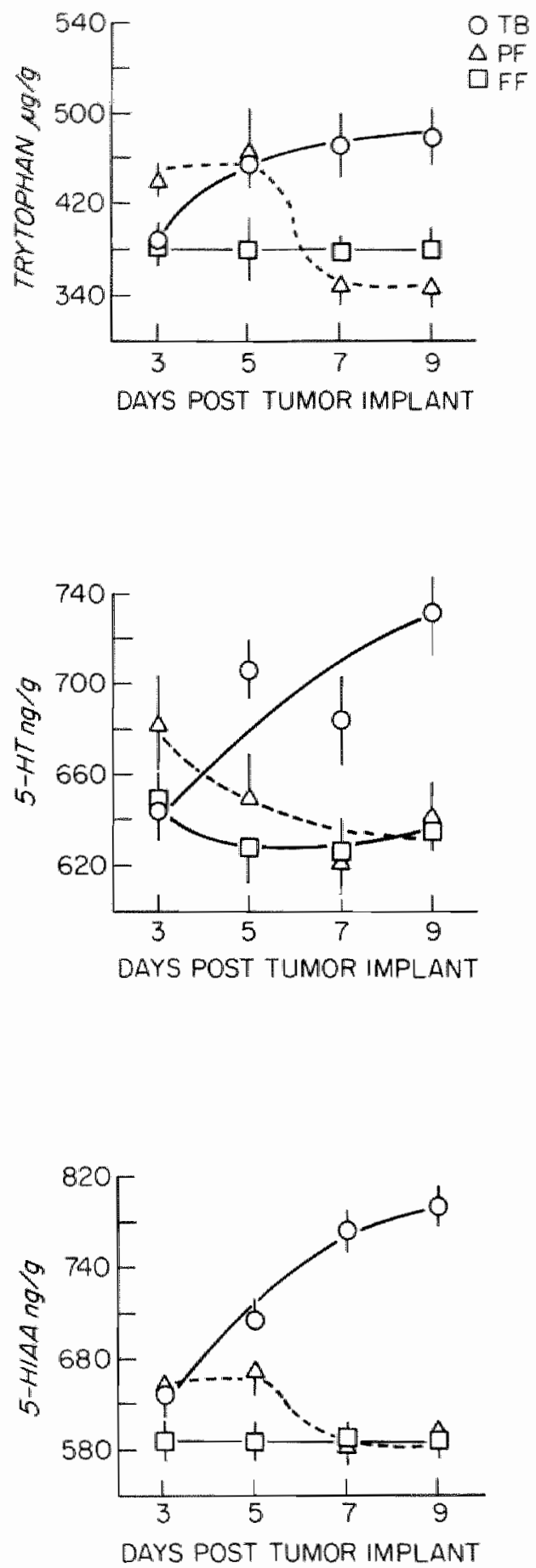

Fig. 4.3.-2 Mean ( \pm SEM) levels of TRP, 5-HT and 5-HIAA on days $3,5,7$ and 9 after the im. injection of $5 \times 10^{4} \mathrm{~W}_{258}$ tumor cells in TB rats, or normal saline in PF and $F F$ control rats. For each data point $n=8$. 


\subsection{Materials and methods}

\section{Design}

Ninety six immature female SD-rats served as subjects and were again divided over 3 groups: a $W_{2 s 6}$ tumor bearing group $(T B, n=32)$, and $P F(n=32)$ and $F F(n=32)$ control groups. Equal numbers $(n=8)$ of each group were sacrificed by decapitation $3,5,7$ and 9 days after tumor inoculation.

\section{Biochemical assays}

Brains were harvested and analyzed according to the techniques described in chapter 2 , for determination of TRP, 5-HT, 5-HIAA, NE and DA levels.

\subsection{Results}

Again a significant $(t=3.23 ; p<0.01)$ decrease in food intake in TB animals was observed 6 days after tumor induction (Fig. 4,3,-1.) while the anorexia intensified across days. Analysis of biachemical data from the brains indicated that changes in central $5-H T$ synthesis and metabolism preceded the development of anorexia in TB rats, paralleling its severity (Fig. 4.3.-2),

Thus, overall analysis of variance indicated changes in $\operatorname{TRP}(F(2,84)=6.43 ; p<0.01), 5-H T$ $(F(2,84)=14.21: p<0.01)$ and 5-HIAA $(F(2,84)=71.36 ; p<0.01)$ as well as significant groups $x$ days interaction for each of these compounds, while no changes in DA and NE were observed. One way ANOVA conducted across the 4 test days indicated significantly increased levels of TRP $(F(3.28)=3.07 ; p<0.05), 5-H T(F(3.28)=5.21 ; p<0.01)$ and $5-H \| A A(F(3,28)=18.00 ; p<0.01)$ in TB groups, while TRP $(F(3.28)=7.60 ; p<0.01)$ and 5 -HIAA $(F(3.28)=3.45 ; p<0.05)$ decreased in the PF groups. Daily comparison of the groups revealed significant group differences in TRP on days $3(F(2,21)=3.61 ; p<0.05), 7(F(2.21)=15.92: p<0.01)$ and $9(F(2.21)=40.45 ; p<0.01)$, in $5-H T$ on days $5(F(2,21)=6.37 ; p<0.01), 7(F(2,21)=4.40 ; p<0.05)$ and $9(F(2,21)=10.34 ; p<0.01)$ and in $5-H I A A$ on days $5(F(2,21)=7.29 ; p<0.01), 7(F(2,21)=45.87 ; p<0.01)$ and $9(F(2,21)=64.69 ; p<0.01)$. Post hac t-tests showed that $T B$ rats had significantly $(p<0.01)$ increased TRP and 5-HIAA levels as compared to PF and FF groups on days 7 and 9 . In addition, 5-HIAA levels were elevated on day $5(p<0.01)$ in the TB group, as compared to the FF rats, while $5-H T$ levels were increased in the TB group as compared to PF and FF groups on days $5(p<0.05), 7(p<0.05)$ and 9 $(p<0.05)$ (Table 4.3.-1).

\subsection{Discussion}

In this experiment it was demonstrated that changes in CNS indole activity occur immediately prior to the onset of anorexia. Thus, rats bearing $W_{256}$ tumors exhibited increased brain 5-HT and 5-HIAA levels on day 5, while significant anorexia was not observed until day 6 . This early change in CNS indoles is the first demonstration that biochemical alterations in putative neurotransmitters known to influence food intake precede the development of cancer anorexia.

The biochemical changes observed in PF animals on days 3 and 5 may be a result of food restriction. Thus, Krieger (1980) observed in food restricted animais (fed at $10 \mathrm{am}$ ) a reversed periodicity pattern of serotonin, resulting in peak levels for serotonin at 12.00 and $8.00 \mathrm{pm}$, whereas ad lib. fed animals displayed $\|$ ower levels at $8.00 \mathrm{pm}$. This change is presumably a result of displacement of plasma TRP from albumin binding sites by non esterified fatty acids, which are increased as a result of lipolytic processes associated with food deprivation, that are possibly a consequence of increased corticasteroid activity. 
Table 4.3.-1 Mean ( I SEM) brain levels of tryptophan (TRP), serotonin (5-HT), 5-hydroxyindoleacetic acid (5-HIAA), norepinephrine (NE) and dopamine (DA) in tumor bearing (TB). pair-ted (PF) and freely teeding (FF) control rats 3,5,7 and 9 days after the injection (im.) of $5 \times 10^{4}$ Walker 256 carcino sarcoma cells in TB animals. At the bottom $p$ values of post hoc t-tests.

\begin{tabular}{|c|c|c|c|c|c|}
\hline & & $\begin{array}{l}\text { Days of sac } \\
3\end{array}$ & 5 & 7 & 9 \\
\hline $\begin{array}{l}\text { TAP } \\
(\mu g / g)\end{array}$ & $\begin{array}{l}\text { TB } \\
\mathrm{PF} \\
\mathrm{FF}\end{array}$ & $\begin{array}{l}3.88 \pm 0.21 \\
4.41 \pm 0.14 \\
3.83 \pm 0.15\end{array}$ & $\begin{array}{l}4.56 \pm 0.20 \\
4.69 \pm 0.37 \\
3.81 \pm 0.26\end{array}$ & $\begin{array}{l}4.73 \pm 0.28 \\
3.49 \pm 0.17^{a} \\
3.79 \pm 0.14^{a}\end{array}$ & $\begin{array}{l}4.79 \pm 0.25 \\
3.47 \pm 0.17^{a} \\
3.80 \pm 0.21^{a}\end{array}$ \\
\hline $\begin{array}{l}\text { 5-HT } \\
(\mathrm{ng} / \mathrm{g})\end{array}$ & $\begin{array}{l}\text { TB } \\
\text { PF } \\
\text { FF }\end{array}$ & $\begin{array}{l}644 \pm 12 \\
682 \pm 21 \\
649 \pm 12\end{array}$ & $\begin{array}{l}707 \pm 14 \\
650 \pm 19^{b} \\
629 \pm 16^{a}\end{array}$ & $\begin{array}{l}684 \pm 20 \\
623 \pm 15^{b} \\
627 \pm 14^{b}\end{array}$ & $\begin{array}{l}732 \pm 19 \\
641 \pm 16^{a} \\
635 \pm 8^{a}\end{array}$ \\
\hline $\begin{array}{l}5 \text {-HIAA } \\
(\mathrm{ng} / \mathrm{g})\end{array}$ & $\begin{array}{l}\text { TB } \\
\text { PF } \\
\text { FF }\end{array}$ & $\begin{array}{l}628 \pm 15 \\
631 \pm 17 \\
589 \pm 18\end{array}$ & $\begin{array}{l}694 \pm 21 \\
649 \pm 21 \\
587 \pm 18^{2}\end{array}$ & $\begin{array}{l}773 \pm 17 \\
586 \pm 18^{a} \\
590 \pm 12^{a}\end{array}$ & $\begin{array}{l}793 \pm 17 \\
592 \pm 9^{a} \\
588 \pm 15^{a}\end{array}$ \\
\hline $\begin{array}{l}\text { NE } \\
(n g / g)\end{array}$ & $\begin{array}{l}\text { TB } \\
P F \\
\text { FF }\end{array}$ & $\begin{array}{l}455 \pm 11 \\
460 \pm 10 \\
458 \pm 14\end{array}$ & $\begin{array}{l}425 \pm 5 \\
450 \pm 10 \\
469 \pm 13\end{array}$ & $\begin{array}{l}479 \pm 15 \\
467 \pm 11 \\
453 \pm 15\end{array}$ & $\begin{array}{l}505 \pm 19 \\
476 \pm 13 \\
484 \pm 11\end{array}$ \\
\hline $\begin{array}{l}\mathrm{DA} \\
(\mathrm{ng} / \mathrm{g})\end{array}$ & $\begin{array}{l}\text { TB } \\
\text { PF } \\
\text { FF }\end{array}$ & $\begin{array}{l}707 \pm 39 \\
717 \pm 46 \\
779 \pm 32\end{array}$ & $\begin{array}{l}770 \pm 15 \\
801 \pm 13 \\
807 \pm 15\end{array}$ & $\begin{array}{l}888 \pm 22 \\
755 \pm 13 \\
797 \pm 23\end{array}$ & $\begin{array}{l}892 \pm 27 \\
844 \pm 19 \\
910 \pm 27\end{array}$ \\
\hline
\end{tabular}

Consequently brain TRP levels, serotonin synthesis and degradation increase (Curzon, 1972; Perez Cruet, 1972; Kantak, 1978). It could be hypothesized, that adaptation to the stress of food restriction would be associated with normalization of plasma corticosteroid levels and non esterified fatty acids, resulting in normal TRP binding to albumin, and consequently normal brain TRP, 5-HT and 5-HIAA levels.

Although some changes in brain norepinephrine and dopamine were observed across these days, no consistent pattern of change in catecholamines, correlated with the onset or severity of anorexia, was noted.

\subsection{Conclusions}

1. Changes in CNS indoleactivity occur immediately prior to the onset of anorexia: rats bearing $W_{256}$ tumors exhibited increased brain 5-HT and 5-HIAA levels on day 5, while significant anorexia was not observed until day 6 .

2. No consistent pattern of change in brain catecholamine concentrations correlated with the onset or severity of cancer anorexia, was observed. 


\section{Chapter 5}

\section{Generality of the observed tryptophan and indoleamine system derangements in anorectic tumor bearing animals}

\subsection{Introduction}

Thus far, derangements in CNS TRP/5-HT levels were observed in only one animal-tumor model, displaying cancer induced anorexia, but no host depletion. To exclude tumor specifity of these changes, and to demonstrate that even more severely malnourished PF animals do not display the changes in TRP and 5-HT seen in TB animals, similar experimental investigations were conducted in the more chronic methyl cholanthrene (MCA) -induced sarcoma (Grant, 1974), obtained in situ from the N.C.I. (Bethesda, Md.). The use of the MCA induced sarcoma model provided prolonged anorexia and substantial host depletion. while also causing more severe depletion of body weight in PF animals. The dissociation of TRP and 5-HT changes in depleted PF animals from those observed in TB animals would demonstrate further support for the hypothesis, suggesting TAP and 5-HT changes as being causative in and not a consequence of the development of cancer anorexia.

\subsection{Materials and methods}

\section{Design}

Thirty two male adult (225-250 g) Fisher 344 rats were divided into 3 groups. In 16 animals MCA tumors (TB) were induced SC. whille an additional 8 rats were paired in weight to the first 8 TB animals and constituted the pair-fed (PF) group. Eight additional rats formed the freely feeding control (FF) group.

The time of sacrifice for all animals was determined by the rapid deterioration of the clinica: condition of some TB animals, being day 33 post tumor implantation.

Twenty four additional Fisher 344 rats, equally divided over 3 groups in a similar experiment (Exp. 2) were sacrificed by decapitation in triplets: one animal from each group. Time of sacrifice for a TB, and its paired PF and FF control animals was in this experiment determined by two consecutive days of foodintake less than $3 \mathrm{~g} / 100 \mathrm{~g} \mathrm{BW}$ by this TB rat.

\section{Dissection}

At sacrifice in experiment 1 , blood and brains were collected for determination of plasma albumin, free and total TRP, amino acids and brain TRP, 5-HT, 5-HIAA, NE and DA lavels. Additionally, tumors were carefully dissected and weighed. One TB animal was lost for biochemical assays because of untimely death.

From all animals in experiment 2, blood was collected at sacrifice for determination of plasma albumin, free and total TRP and AA, whille livers were removed and quadriceps muscles were dissected to allow measurement of wet and dry weights and soluble protein contents. Again, one animal was lost for muscle and liver analyses.

\section{Biachemical assays}

All biochemical assays and tissue determinations mentioned above were performed according to the standard techniques described in chapter 2 , or chapter 3 , section 3.2 


\subsection{Results}

Implantation of the methylcholanthrene induced sarcoma in adult male Fisher 344 rats in experiment 1 resulted in significantly $(t=2.26: 0<0.05)$ decreased food intake by TB animals as compared to FF rats by day 23 following tumor inoculation (Fig. 5.3.-1). Tumors were palpable $9-13$ days after the injection of tumor cells.

The absence of body weight gain in PF rats from the onset of anorexia (Fig. 5.3.-2) emphasizes that the daily food consumption by the TB group was not sufficient to support normal growth. The degree of cachexia in this tumor model is indicated by body weight differences on the day of sacrifice. Thus, although mean body weight of TB rats was $250 \pm 3 \mathrm{~g}$. subtraction of the tumor weights $(52 \pm 4 \mathrm{~g})$ yielded a net body weight of $\mathrm{TB}$ rats $(198 \pm 5 \mathrm{~g})$. that was less than that of PF $(215 \pm 7 \mathrm{~g})$ or FF $(250 \pm 4 \mathrm{~g})$ groups.

The presence of cachexia is further demonstrated by changes in muscle tissue (Table $5.3,-1$ ). Both wet and dry weights were decreased in the TB group as compared to both control groups. However the PF animals also lost a significant amount of lean body mass, as demonstrated by lower wet and dry weights of tissue and a stable amount of tissue water. Muscle tissue of TB animals contained significantly more water. The loss of lean body tissue in PF animals occurred apparently at the cost of the non-water solublie protein or fat fraction, while in TB animals an absolute amount of these water soluble proteins must have been lost.

Analysis of changes in liver weight and liver composition yielded significantly lower wet and dry weights in the presence of a stable water fraction in the PF group (Table 5.3.-2). The increased water content of liver also resulted in significant lower dry weights in the TB group, as compared to the FF group. The observed loss of lean tissue in TB and PF animals did not apparantly result in alterations of the relative protein content, though the absolute amounts of water soluble proteins was diminished in these groups.

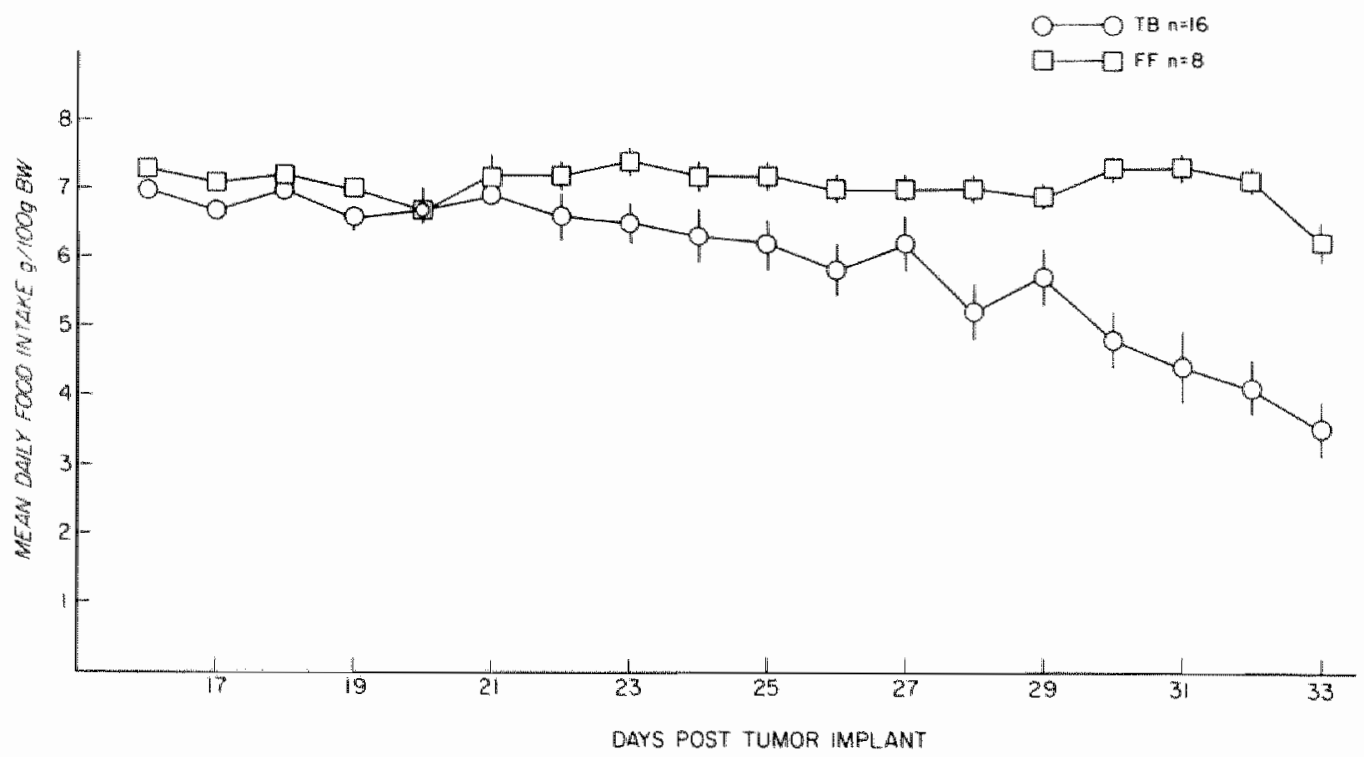

Fig. 5.3.-1 Mean ( 1 SEM) daily food intake of tumor bearing (TB) and freely feeding (FF) control rats. On day 0 TB rats were injected (sc.) with $2 \times 10^{5}$ vilable MCA sarcoma cells, while FF rats receiwed a sham injection normal saline.

Significant $(p<0,05)$ anorexia was observed on day 23 and increased in severity until sacrifice on day 33 . 


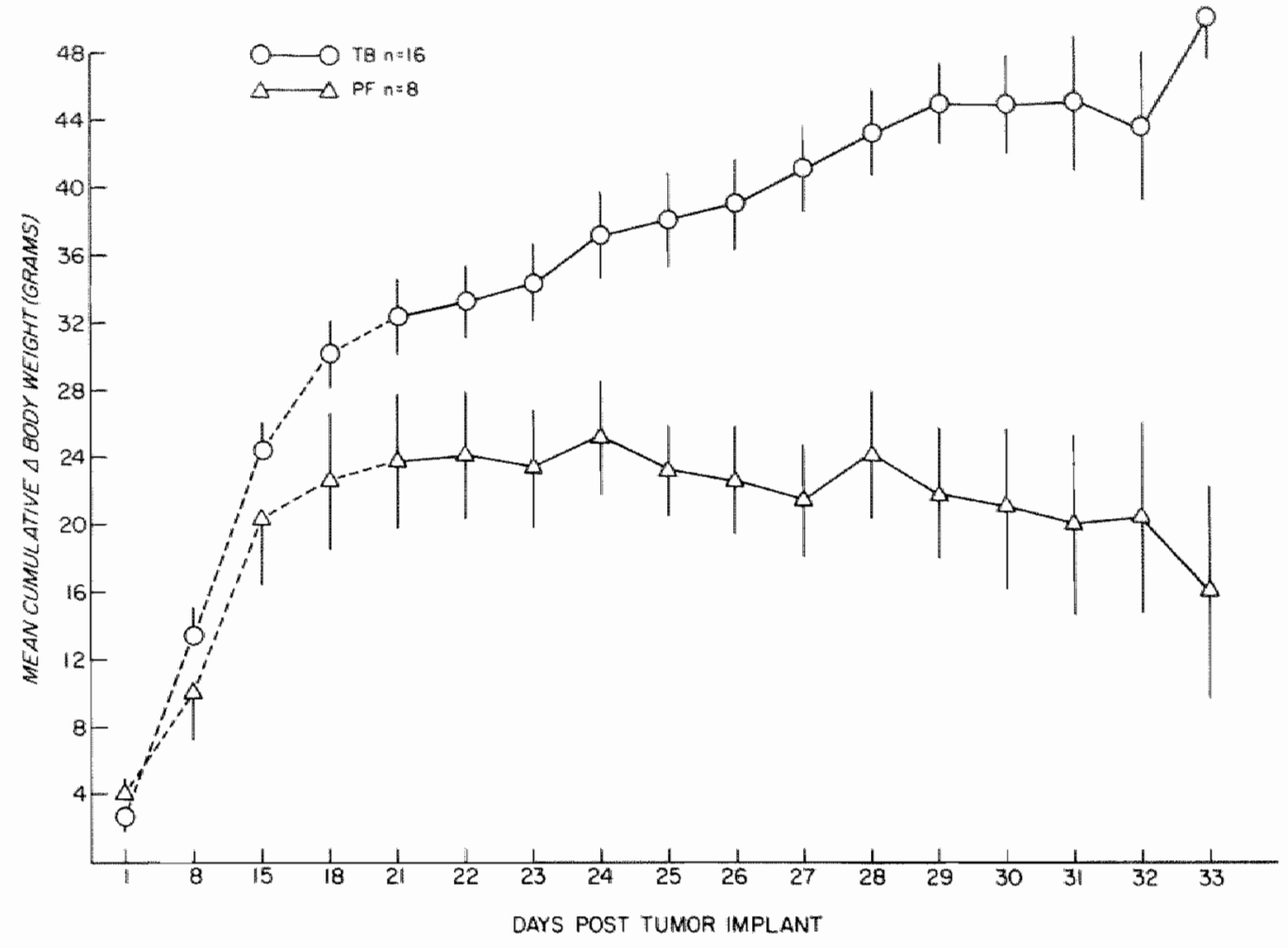

Fig. 5.3.-2. Mean ( \pm SEM) change in body weight of tumor bearing (TB) and pair-fed control (PF) rats during 33 days of tumor growth. Body weight on day 0 , when TB were inoculated with tumor. was reference weight.

Table 5.3.-1 Mean ( \pm SEM) weights, water content and soluble protein content of quadriceps muscles from tumor bearing (TB), pair-fed (PF) and freely feeding (FF) control rats, in experiment 2. Between parenteses results of one-way ANOVA's of each item, at the bottom $p$ values of post hoc t-tests.

\begin{tabular}{|c|c|c|c|c|}
\hline Muscles & $\begin{array}{l}\text { TB } \\
(n=7)\end{array}$ & $\begin{array}{l}P F \\
(n=8)\end{array}$ & $\begin{array}{l}F F \\
(n=8)\end{array}$ & \\
\hline $\begin{array}{l}\text { Wet weight } \\
\text { (grams) }\end{array}$ & $1.787 \pm 0.095$ & $2.701 \pm 0.057^{a}$ & $3.142 \pm 0.056^{\mathrm{ad}}$ & $\begin{array}{l}(F(2,20)=88.25 \\
p<0.001)\end{array}$ \\
\hline $\begin{array}{l}\text { Dry weight } \\
\text { (grams) }\end{array}$ & $0.445 \pm 0.034$ & $0.714 \pm 0.018^{a}$ & $0.835 \pm 0.023^{\mathrm{ad}}$ & $\begin{array}{l}(F(2,20)=65.56 \\
p<0.001)\end{array}$ \\
\hline $\begin{array}{l}\text { Water content } \\
(\%)\end{array}$ & $75.29 \pm 0.68$ & $73.58 \pm 0.20^{c}$ & $73.45 \pm 0.34^{c}$ & $\begin{array}{l}(\mathbb{F}(2,20)=5.48 \\
p<0.025)\end{array}$ \\
\hline $\begin{array}{l}\text { Protein } \\
\text { (mg/g dry weight) }\end{array}$ & $344.0 \pm 8.0$ & $340.1 \pm 3.9$ & $287.8 \pm 13.1^{b d}$ & $\begin{array}{l}(\mathbb{F}(2,20)=13.45 ; \\
p<0.001)\end{array}$ \\
\hline
\end{tabular}

$a=p<0.001$ vs TB; $b=p<0.01$ vs TB; $c=p<0.05$ vs TB; $d=p<0.01$ vs PF 
Table 5.3.2 Mean (1. SEM) weights, water content and soluble protein content of livers from tumor bearing $T B$, pair-fed (PF) and freely feeding (FF) control rats, in experiment 2. Between parenteses results of one-way ANOWA's of each item, at the bottom p values of post hoc t-tests.

\begin{tabular}{|c|c|c|c|c|}
\hline Livers & $\begin{array}{l}T B \\
(n=7)\end{array}$ & $\begin{array}{l}\text { PF } \\
(n=8)\end{array}$ & $\begin{array}{l}\mathrm{FF} \\
(n=8)\end{array}$ & \\
\hline $\begin{array}{l}\text { Wet weight } \\
\text { (grams) }\end{array}$ & $8.682 \pm 0.731$ & $6.868 \pm 0.192^{b}$ & $10.137+0.293^{c}$ & $\begin{array}{l}(F(2.20)=14.79 \\
p<0.001)\end{array}$ \\
\hline $\begin{array}{l}\text { Dry weight } \\
\text { (grams) }\end{array}$ & $2.284 \pm 0.169$ & $2.071 \pm 0.071$ & $3.052 \pm 0.082^{a c}$ & $\begin{array}{l}(F(2.20)=23.56 \\
p<0.001)\end{array}$ \\
\hline $\begin{array}{l}\text { Water content } \\
(\%)\end{array}$ & $73.58 \pm 0.29$ & $69.88 \pm 0.30^{a}$ & $69.84 \pm 0.59^{9}$ & $\begin{array}{l}(F(2,20)=24.14 \\
p<0.001)\end{array}$ \\
\hline $\begin{array}{l}\text { Protein } \\
\text { (mg/g dry weight) }\end{array}$ & $794.4 \pm 17.1$ & $849 . \pm 21.0$ & $788.1 \pm 14.1$ & $\begin{array}{l}(F(2,20)=3.78 \\
p<0.05)\end{array}$ \\
\hline
\end{tabular}

$a=p<0.001$ vs TB; $b=p<0.05$ vs TB; $c=p<0.001$ vs PF

Table 5.3.-3 Mean ( I SEM) levels of plasma albumin, total tryptophan (TRP), free TRP, ratio free TRP/sum neutral amino acids ( $N$ NAA) and brain TRP, serotonin (5-HT), 5-hydroxyindoleaceli acid (5-HIAA), norepinephrine (NE) and dopamine (DA) in tumor bearing (TB), pair-fed (PF) and freely feeding (FF) control rats 33 days after induction of MCA tumors (Exp. 1).

Between parenteses $F$ and $p$ values of analysis of variance of each factor, at the bottom $p$ values of post hoc t-tests.

\begin{tabular}{|c|c|c|c|c|}
\hline Plasma & $\begin{array}{l}\text { TB } \\
(n=15)\end{array}$ & $\begin{array}{l}\text { PF } \\
(n=8)\end{array}$ & $\begin{array}{l}F F \\
(n=8)\end{array}$ & \\
\hline Albumin $(g / d l)$ & $3.66 \pm 0.21$ & $4.52+0.08^{\circ}$ & $4.36 \pm 0.06^{c}$ & $(F(2,28)=6.28 ; p<0.01)$ \\
\hline Total TRP $(\mathrm{nmol} / \mathrm{ml})$ & $77.6 \pm 4.6$ & $100.1 \pm 7.9$ & $114.5 \pm 5.7^{\oplus}$ & $(F(2,28)=11.19 ; p<0.001)$ \\
\hline Free TRP (nmol/ml) & $19.5 \pm 1.4$ & $14.6 \pm 0.5$ & $17.1 \pm 2.0$ & n.s. \\
\hline Ratio free TRP/INAA & $0.025 \pm 0.002$ & $0.020 \pm 0.001$ & $0.021 \pm 0.002$ & n.s. \\
\hline
\end{tabular}

Brain

\begin{tabular}{lcccl}
\hline TRP $(\mu \mathrm{g} / \mathrm{g})$ & $3.54 \pm 0.21$ & $2.61 \pm 0.12^{\mathrm{b}}$ & $2.41 \pm 0.15^{\mathrm{b}}$ & $(\mathrm{F}(2,28)=10.14 ; \mathrm{p}<0.001)$ \\
$5-\mathrm{HT}(\mathrm{ng} / \mathrm{g})$ & $775 \pm 18$ & $663 \pm 23^{\mathrm{b}}$ & $646 \pm 19^{a}$ & $(\mathrm{~F}(2,28)=13.55 ; \mathrm{p}<0.001)$ \\
$5-H I A A(\mathrm{ng} / \mathrm{g})$ & $597 \pm 16$ & $454 \pm 9^{\mathrm{a}}$ & $418 \pm 6^{\mathrm{a}}$ & $(\mathrm{F}(2,28)=31.24 ; \mathrm{p}<0.001)$ \\
NE $(\mathrm{ng} / \mathrm{g})$ & $533 \pm 16$ & $467 \pm 9^{\mathrm{b}}$ & $523 \pm 10$ & $(\mathrm{~F}(2,28)=6.17 ; \mathrm{p}<0.01)$ \\
DA $(\mathrm{ng} / \mathrm{g})$ & $1056 \pm 33$ & $936 \pm 23^{\mathrm{b}}$ & $1101 \pm 29$ & $(\mathrm{~F}(2,28)=6.00 ; \mathrm{p}<0.01)$
\end{tabular}

$a=p<0.001$ vs TB; $b=p<0.01$ vs TB; $c=p<0.05$ vs TB 
Table 5.3.-4 Mean ( \pm SEM) levels of plasma aibumin, total tryptophan (TRP). Uree TRP. ratio free TRP/ENAA of tumor bearing (TB), pair-fed (PF) and freely feeding (FF) control rats in the second MCA experiment.

\begin{tabular}{|c|c|c|c|c|}
\hline & $\begin{array}{l}\text { TB } \\
(n=8)\end{array}$ & $\begin{array}{l}P F \\
(n=8)\end{array}$ & $\begin{array}{l}F F \\
(n=8)\end{array}$ & \\
\hline Albumin g/dl & $1.94 \pm 0.13$ & $2.27 \pm 0.05^{c}$ & $2.37 \pm 0.05^{b}$ & $(F(2,21)=7.95 ; p<0.01)$ \\
\hline Total TRP $\mathrm{nmol} / \mathrm{ml}$ & $63.3 \pm 4.8$ & $100.2 \pm 5.7^{\mathrm{a}}$ & $119.6 \pm 3.7^{\mathrm{a}}$ & $(F(2,21)=40.73 ; p<0.01)$ \\
\hline $\begin{array}{l}\text { Free TRP nimol/ml } \\
\text { Ratio free }\end{array}$ & $22.8 \pm 3.8$ & $14.5 \pm 0.8^{c}$ & $19.5 \pm 1.3$ & $(F(2,21)=3.56 ; p<0.05)$ \\
\hline TRP/INAA & $0.024 \pm 0.005$ & $0.016 \pm 0.001$ & $0.019 \pm 0.002$ & n.s. \\
\hline
\end{tabular}

$a=p<0.001$ vs TB; $b=0<0.01$ vs. TB; $c=p<0.05$ vs TB

Biochemical analyses (Tables 5.3.-3 and 5.3.-4) revealed simillar, though not identical, changes in 5-HT metabolism as observed in the $W_{256}$ model. Thus, plasma albumin and total TRP were significantly decreased, while plasma free TRP was significantly ellevated in TB rats as compared to PF animals only in experiment 2 . The ratio of plasma free TRP to the sum of NAA tended to be increased in TB animals, but this observation was not statistically significant.

Brain levels of TRP, 5-HT and 5-HIAA were significantly elevated in TB rats as compared to either control group, while NE and DA levels were significantly decreased in the PF animals.

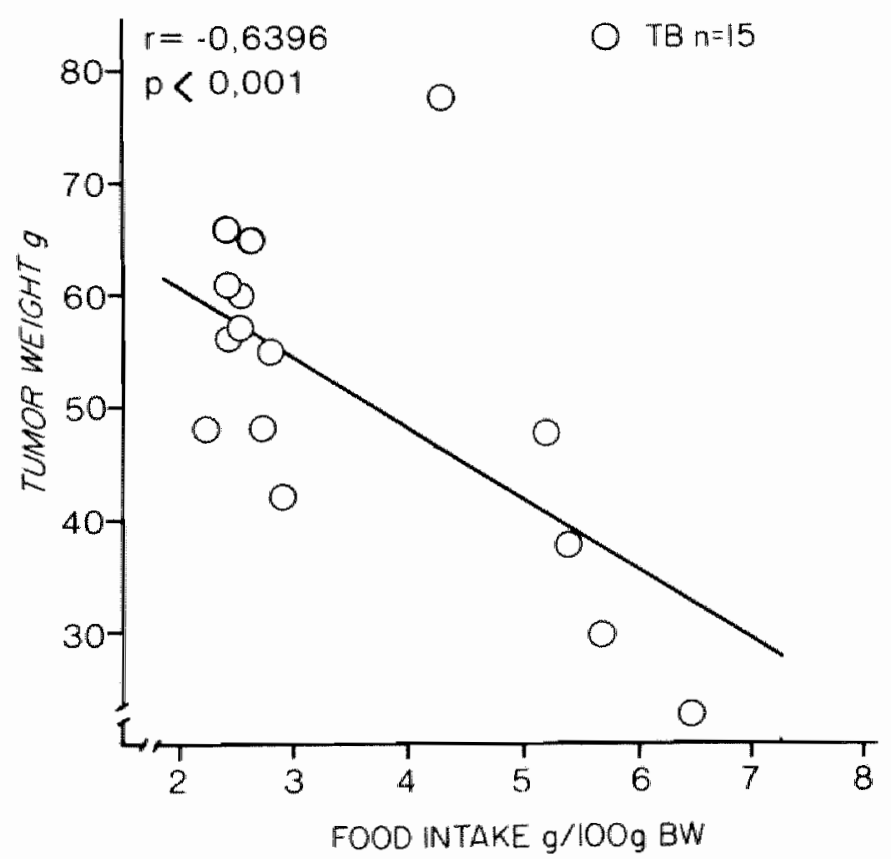

Fig. 5.3.-3 Correlation of food intake ( $9 / 100 \mathrm{~g} \mathrm{BW}$ ) with tumor weights (g) in 15 MCA tumor bearing animals (Exp. 1). 


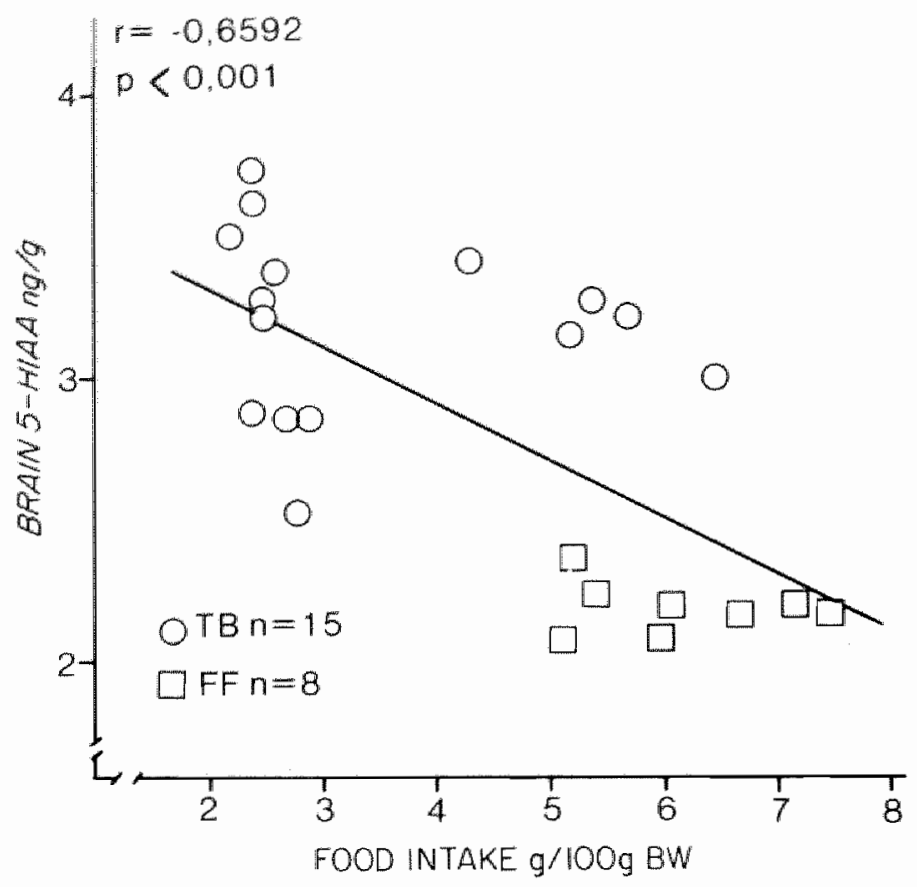

Fig. 5.3.-4. Correlation of food intake (g/100 g BW) and brain 5-HIAA content (nmol/g) in $15 \mathrm{MCA}$ tumor bearing (TB) and 8 freelly feeding (FF) control animals. (Exp. 1).

Furthermore, significant negative correlations were observed between food intake on the day of sacrifice and tumor weight $(r=-0.6396$; Fig. 5.3.-3) and brain 5-HIAA $(r=-0.6592$; Fig. 5.3.-4). The ratio plasma free TRP/LNAA was significantly $(p<0.001)$ correlated with brain TRP ( $r=0.6186 ;$ Fig. 5.3. -5$)$, which correlated well with brain 5-HT $(r=0.7105 ;$ Fig. 5.3.-6) and 5-HIAA $(r=0.7094 ;$ Fig. 5.3,-7). Brain 5-HT levels were also significantly $(p<0.001)$ correlated with brain 5-HIAA levels $(r=0.6444$; Fig. 5.3.-8).

\subsection{Discussion}

These experiments yield behavioral data very similar to the reports by Popp (1980), characterizing this tumor model. llt is also demonstrated that central indoleamine derangements in cancer anorexia are not an isolated phemomenon associated with the acute $W_{256}$ tumor model. Thus, elevations in brain TRP, 5-HT and 5-HIAA were present in MCA TB animals, demonstrating a prolonged anorexia as well as cachexia and therefore probably more closely reflecting the clinical course of this disease. Furthermore, the absence of such changes in malnourished PF animals suggests that the changes in 5-HT activity are tumor specific and not a result of malnutrition.

Some variation in the ratio plasma free TRP/INAA in TB rats prohibited these data becoming significantly different from either control group. However, this ratio was somewhat elevated in TB rats and correlated well with brain TRP values per individual animal.

The cause for plasma free TRP levels not to rise, and plasma total TRP not to fall as much in a chronic model of cancer anorexia as in an acute model remains uncertain. It could be 

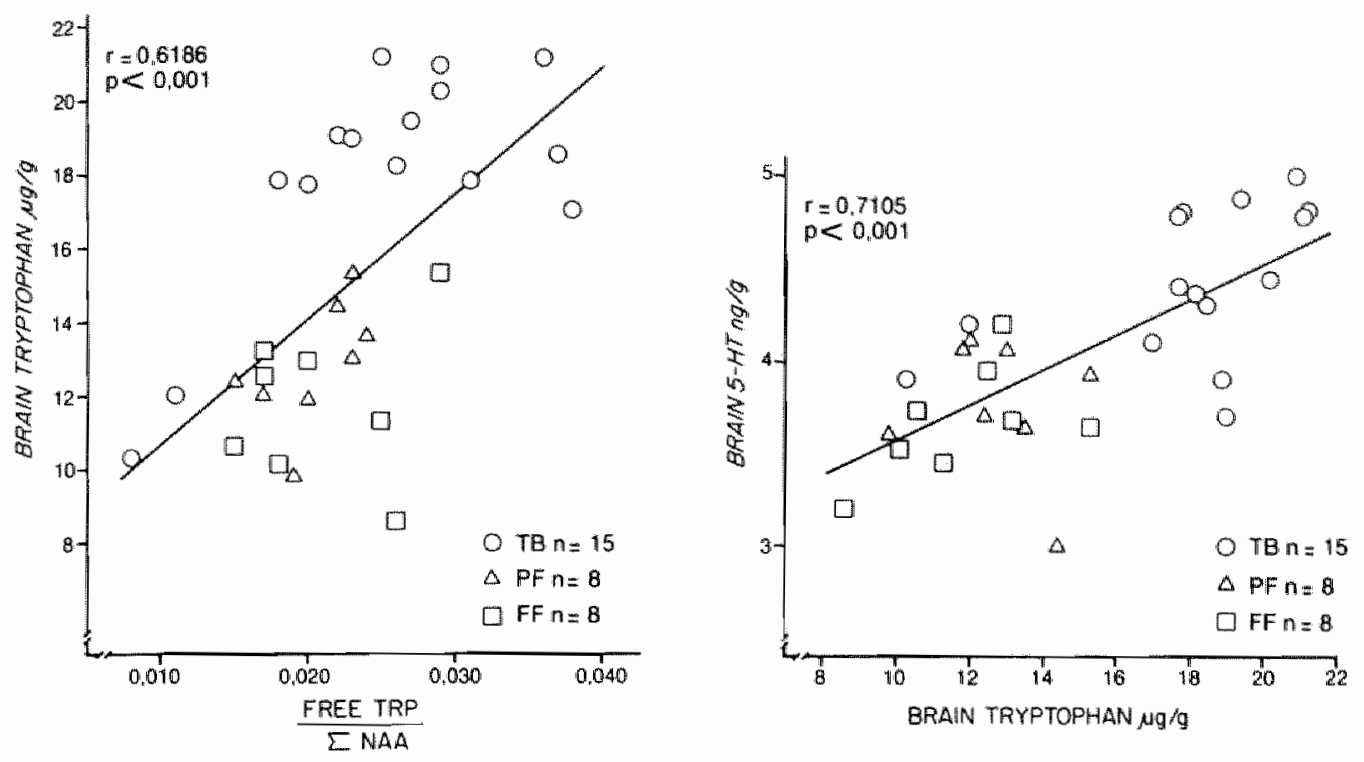

Fig. 5.3.-5 Correlation of the ratio plasma free TRP over the sum of NAA with brain TRP levels $(\mu \mathrm{g} / \mathrm{g})$ in all animals in Exp. 1.

Fig. 5.3.-6 Correlation of brain TAP $(\mu \mathrm{g} / \mathrm{g})$ with brain $5-\mathrm{HT}(\mathrm{ng} / \mathrm{g})$ in all animals in Exp. 1.
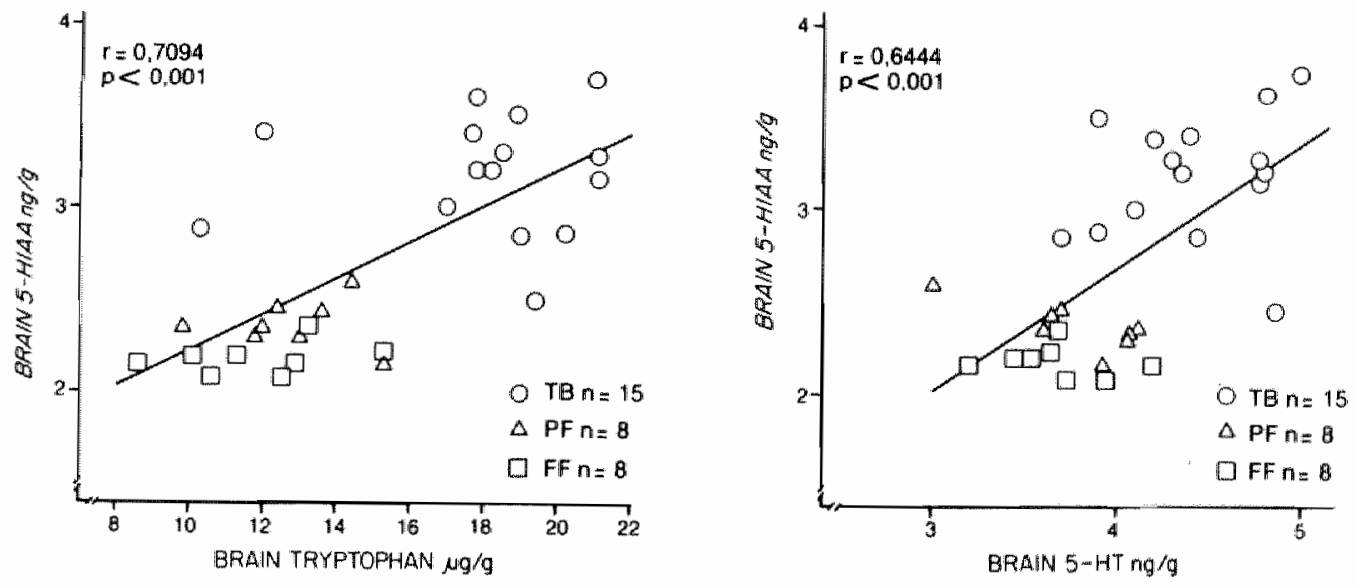

Fig. 5.3.-7 Correlation of brain TRP $(\mu \mathrm{g} / \mathrm{g})$ with brain 5-HIAA $(\mathrm{ng} / \mathrm{g})$ in all animals in Exp. 1 .

Fig. 5.3.-8 Correlation of brain 5-HT (ng/g) with brain 5-HIAA (ng/g) in all animals in Exp. 1. 
speculated that depletion of fat stores causes plasma FFA to fall, thus allowing binding of TRP to a greater exlent, and consequenty limiting the degradation of this amino acid as has been theorized in chapter 1, section 1.4. It should be emphasized, however, that no sufficient data are available on what factors control TAP degradation in general, and more specifically in cancer.

These results also confirm reports by Friedman (1972) and Pardridge (1979). that the Km of the rate limiting enzyme in serotonin synthesis. TRP hydroxylase, is equal to or greater than the intracellular TRP concentrations, resulting in precursor supply (TRP) influencing the rate of $5-H T$ synthesis and degradation.

Considering the reported role of catecholamines in modulating feeding (Marshall, 1974 ; Leibowitz, 1976; Panksepp. 1979) it is of considerable interest that TB rats did not exhibit the decreased amines observed in PF animals. Thus, adrenergic systems may also be involved in the genesis of cancer anorexia by falsely signalling satiety or falling to adequately signal hunger in the TB animal.

The observed body weight loss in TB and PF groups may result from different causes, as has been pointed out by others (Lundhoim, 1980; Popp, 1980). Thus, in this tumor model body water is increased in TB animalls, probably due to decreased plasma oncotic pressure, and wasting of lean body lissue is most apparent in peripheral muscle tissue. The weight and water content of liver tissue of TB animals in these experiments was not different from that of PF animals even though TB animals exhibited a greater degree of cachexia. This observation may be explained, in part, by increased protein synthesis activity, as has been observed in mice (Lundholm, 1979) and in humans (Lundholm, 1978).

\subsection{Conclusions}

1. The MCA tumor model displays prolonged anorexia: food intake by TB rats was significantly reduced during approximately one third of the time course of MCA tumor growth, uitimately resulting in death of the tumor host.

2. The MCA tumor model displays significant cachexia: food intake by TB rals was insufficient to support normal growth in PF animals, and net bady weights of TB rats were less than those of PF or FF groups.

3. The cachexia in this MCA tumor model is best observed in muscle tissue, revealing after analysis increased water contents and loss of lean tissue, also at the cost of the water soluble protein fraction.

Changes in liver tissue of TB animalls are paralleled by changes in PF animals, but may show compared to the more severe cachexia in TB animals, signs of a relative lack of adaptation to starvation

4. Changes observed in TRP and 5-HT levels in MCA TB animals were similar to the ones observed in the $W_{236}$ tumor model. The absence of similar changes in the significantly malnourished PF animals suggests once more that these changes are causative in, rather than a consequence of the development of cancer anorexia. Thus, increased brain 5-HT activity associated with cancer anorexia appears to be a general phenomenon, being readily demonstrated within at least two anorexia-producing tumor lines.

5. Biochemical values of TRP and 5-HT in this tumor model follow patterns that are consistent with reported mechanisms regarding TRP transport into the brain, and 5-HT synthesis and degradation.

6. Catecholaminergic systems may also be involved in the genesis of prolonged anorexia by falsely signalling satiety or failing to adequately signal hunger in the TB animal. 


\section{Chapter 6}

\section{Regional brain serotonergic activity in tumor induced anorexia, studied in two tumor models}

\subsection{Introduction}

Brobeck and coworkers (1943) were the first to suggest the hypothalamus as an integrating area in the control of feeding behavior, based on their observation that lesions in the medial hypothalamus, especially ventrowlateral to the ventromedial nucleus, produced overeating and obesity. About 8 years later they defined earlier incidental abservations by Hetherington and Ranson (1940) of decreased food intake afler hypothalamic lesions, and found, that lesions to the lateral part of the lateral hypothalamus result in aphagia and adipsia (Amand. Brobeck, 1951). In more recent years much research indicated that brain neuronal circuits rather than discrete nuclei alone regulate ingestive behavior (Grossman, 1980; Panksepp. 1979).

The serotonergic component of the brain that is presumably involved in the regulation of ingestive behavior, arises in the lower brain stem and ascends through the hypothalamus (Dahiström, 1965). Since Knott and Curzon (1974) have also found evidence that metabolism of 5-HT may vary by region, it became important to investigate the regional distribution of TRP and indole activity in the brains of rats, displaying anorectic behavior under influence of $W_{256}$ or MCA tumors.

\subsection{Materials and methods}

\section{Design}

Forty eight rats served as subjects across 2 experiments: 24 young female SD rats were divided into 3 groups: TB $(n=8)$, inoculated with the $W_{256}$ tumor, $P F(n=8)$ and $F F(n=8)$. An additional group of 24 adult male Fisher 344 rats was divided in the same way, the TB group being inoculated with the MCA tumor.

Animals in the $W_{256}$ experiment were sacrificed on day 9 post tumor implant, while the rats in the MCA experiment were sacrificed in groups of 3 : a TB rat with its PF and FF controls. Moment of sacrifice was determined by food intake less than $3 \mathrm{~g} / 100 \mathrm{~g} \mathrm{BW}$ for two consecutive days by this TB animal.

The criteria for sacrificing in the MCA experiment made redefinition of anorexia necessary. The condition was considered present, when a TB rat ate for two consecutive days $80 \%$ or less of the average $24 \mathrm{hr}$ food consumption during the first 5 days of the experiment.

\section{Dissection}

After decapitation, brains were removed and rapidly frozen in liquid nitrogen after dissection in a manner similar to the one described by Glowinski and lversen (1966) with the following exceptions:

1. Mesencephalon and diencephalon were differentiated by a vertical cut just anterior to the superior colliculus and:

2. A smaller hypothalamic area was dissected and defined as tissue taken to a depth of approximately $2.5 \mathrm{~mm}$ localized from the optic chiasma to the posterior mamillary area and bounded laterally by the choroid fissure. 
Each brain reglon was weighed before fluorometric assay of TRP, 5-HT and 5-HIAA.

\subsection{Results}

Behawioral data in both experiments reflected our previous findings, in that the $W_{25}$ tumor bearing animals displayed significant anorexia 4 days prior to sacrifice, while MCA TB rats were anorectic for an average of 10 days before sacrifice. The dissection procedure was constant in our hands, as ewidenced by consistent relative weights per region in both experiments. Comparison with data reported by Glowinski and lversen (1966), reweals that the region we dissected as hypothalamus was smaller (Table 6.3.-1). Tryptophan in control animals within both experiments was found to be distributed fairly evenly through the various brain regions with exception of a slightly higher concentration in the hypothalamic regions. This finding is consistent with previous descriptions (Knott, 1974, Cummings, 1976).

Serotonin in the control animals was present in concentrations and distributions comparable to the observations reported by others (Knott, 1974, Cummings, 1976). The 5-HIAA control values showed, like 5-HT, regional differences. Again distributions as well as concentrations compared well with the values reported in the above quoted literature.

Analysis of variance of assay results in experimental animals in the $W_{256}$ tumor model revealed significant elevations of TRP in the hypothalamus $(p<0.01)$, corpus striatum $(p<0.05)$, mesencephalon $(p<0.02)$, diencephalon $(p<0.01)$, cerebellum $(p<0.01)$ and cortex $(p<0.01)$ of TB rats. $5-H T$ was significantly increased in TB rats in the diencephalon $(p<0.05)$ and cerebellum ( $p<0.01)$, while significantly elevated 5 -HIAA values in these animals were observed in the diencephalon $(\rho<0.01)$, hippocampus $(p<0.01)$, pons/medulla $(p<0.05)$, cerebellum $(\rho<0.01)$ and cortex $(p<0.05)$ (Fig. 6.3.-1).

The ratio of the metabolite to its neurotransmitter has recently been proposed as an index of neuronal activity for catecholaminergic neurons (Melamed, 1980; Hefti, 1980). Thus, calculation of the ratio of 5-HIAA to 5-HT would reflect serotonergic neuronal activity, provided that $5-H T$ concentrations remain unchanged, or increase to a smaller degree than 5-HIAA levels do.

Table 6.3.-1 Mean wet weight ( \pm SEM or SD, grams) (percent of total weight) of each brain section of TB and control animals $(n=24)$ in both tumor models, compared with results reported by Glowinski and Iversen (J. Neurochem. 13; 655-669, 1966).

$$
W_{256} \text { tumor MCA Glowinski/Iversen }
$$

\begin{tabular}{lllllll} 
Brain Area & $(\bar{x} \pm S E M)$ & $\%$ & $(\bar{x} \pm S E M)$ & $\%$ & $(\bar{x} \pm S D)$ & $\%$ \\
\hline Hypothalamus & $0.027 \pm 0.001$ & $(2.1)$ & $0.033 \pm 0.001$ & $(2.0)$ & $0.110 \pm 0.014$ & $(6.0)$ \\
Corpus Striatum & $0.126 \pm 0.006$ & $(9.7)$ & $0.168 \pm 0.004$ & $(10.0)$ & $0.102 \pm 0.017$ & $(5.6)$ \\
Mesencephalon & $0.084 \pm 0.003$ & $(6.5)$ & $0.127 \pm 0.003$ & $(7.6)$ & $0.146 \pm 0.010$ & $(8.0)$ \\
Diencephalon & $0.155 \pm 0.005$ & $(12.0)$ & $0.246 \pm 0.007$ & $(14.6)$ & & \\
Hippocampus & $0.089 \pm 0.003$ & $(6.9)$ & $0.134 \pm 0.003$ & $(8.0)$ & $0.151 \pm 0.022$ & $(8.2)$ \\
Pons/Medulla & $0.138 \pm 0.001$ & $(10.6)$ & $0.196 \pm 0.002$ & $(11.7)$ & $0.252 \pm 0.024$ & $(13.8)$ \\
Cerebellum & $0.197 \pm 0.003$ & $(15.2)$ & $0.242 \pm 0.003$ & $(14.4)$ & $0.267 \pm 0.022$ & $(14.6)$ \\
Cortex & $0.480 \pm 0.009$ & $(37.0)$ & $0.535 \pm 0.007$ & $(31.8)$ & $0.804 \pm 0.058$ & $(43.9)$ \\
\hline
\end{tabular}



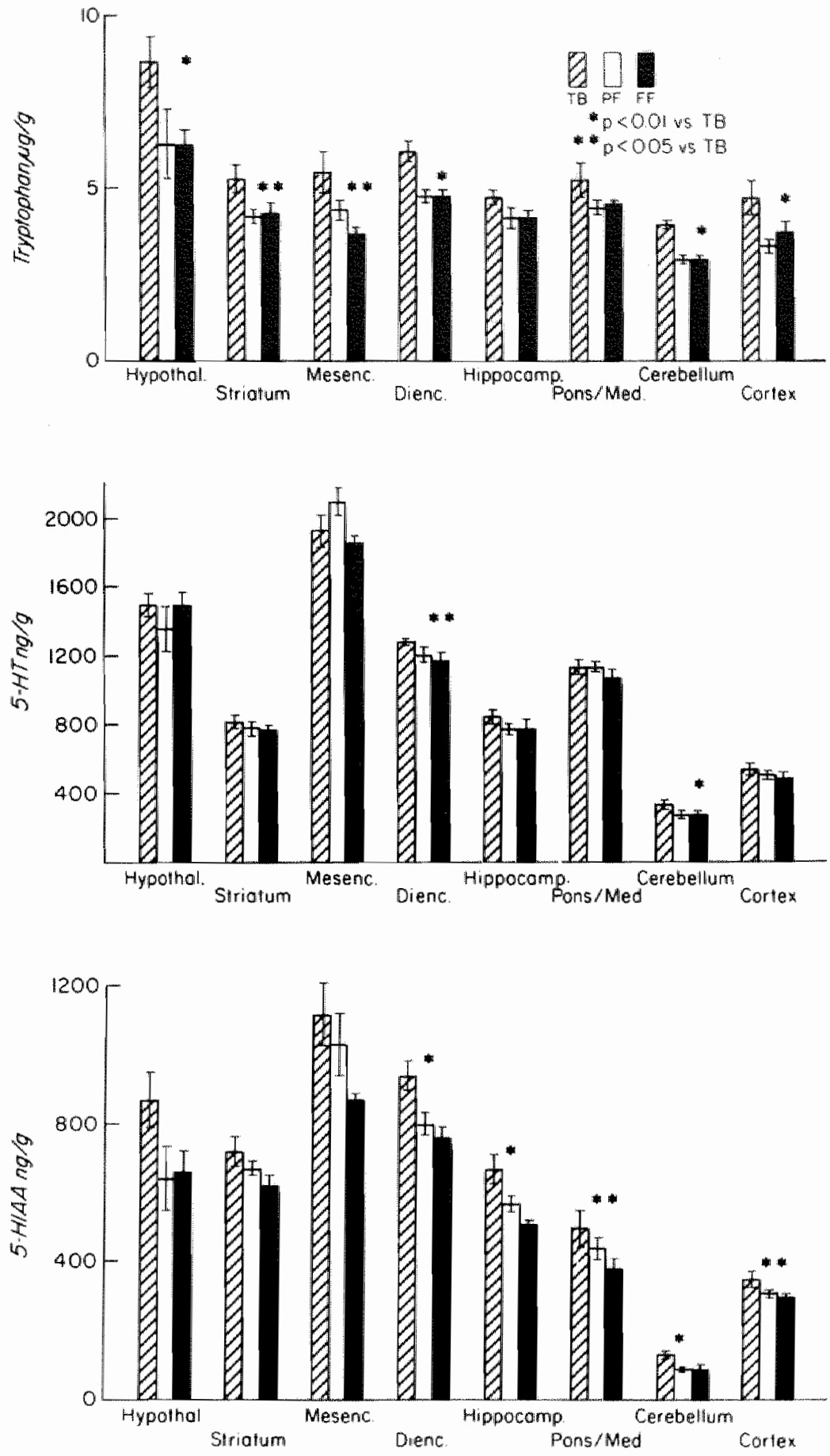

Fig. 6.3.-1 Mean (I SEM) levels of TRP. 5 HT and 5-HIAA in brain regions of TB, and PF and FF control rats. The rats were sacrificed 9 days after the injection (in.) of $5 \times 10^{4}$ viable $W_{25}$ tumor cells in $T B$ animals. For each group in each region $n=8$.

43 

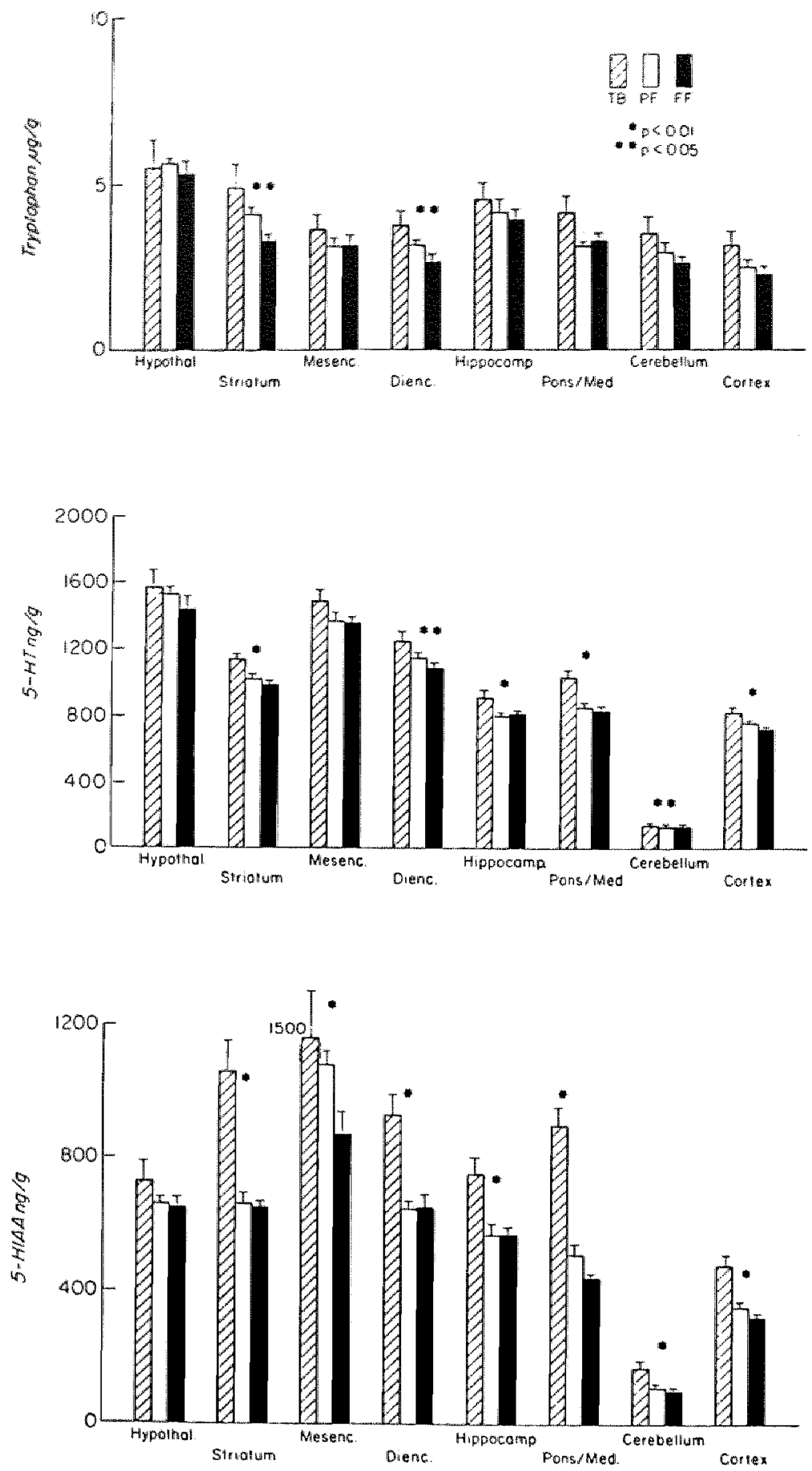

Fig. 6.3.-2 Mean ( 1 SEM) levels of TRF, 5-HT and 5-HIAA in brain regions of TB, PF and FF rats The rats were sacrificed after 2 days of food intake less than $3 \mathrm{~g} / 100 \mathrm{~g}$ BW by TB animals, implanted sc. with MCA tumors. For each group in each region $n=8$. 
Table 6.3.-2 Mean ( I SEM) brain regional ratio of 5-HIAA to 5-HT in Wast tumor bearing (TB), and PF and FF control rats. At the bottom $p$ values of post hoc t-tests.

\begin{tabular}{|c|c|c|c|}
\hline Brain Area & $\begin{array}{l}\text { TB } \\
(n=8)\end{array}$ & $\begin{array}{l}\text { PF } \\
(n=8)\end{array}$ & $\begin{array}{l}F F \\
(n=8)\end{array}$ \\
\hline Hypothalamus & $0.558 \pm 0.029$ & $0.419 \pm 0.044^{b}$ & $0.422 \pm 0.030^{\circ}$ \\
\hline Corpus Striatum & $0.866 \pm 0.035$ & $0.866 \pm 0.040$ & $0.801 \pm 0.039$ \\
\hline Mesencephalon & $0.585 \pm 0.048$ & $0.487 \pm 0.024$ & $0.470 \pm 0.013^{b}$ \\
\hline Diencephalon & $0.733 \pm 0.023$ & $0.661 \pm 0.017^{b}$ & $0.647 \pm 0.018^{\text {a }}$ \\
\hline Hippocampus & $0.765 \pm 0.047$ & $0.740 \pm 0.036$ & $0.693 \pm 0.057$ \\
\hline Pons/Medulla & $0.484 \pm 0.061$ & $0.381 \pm 0.024$ & $0.361+0.035$ \\
\hline Cerebellum & $0.396 \pm 0.029$ & $0.316 \pm 0.013^{b}$ & $0.311 \pm 0.027$ \\
\hline Cortex & $0.663 \pm 0.043$ & $0.621 \pm 0.023$ & $0.600 \pm 0.017$ \\
\hline
\end{tabular}

$a=p<0.01$ vs TB; $b=p<0.05$ vs TB

Analysis of variance of this ratio in TB, PF and FF rats, calculated for each region, showed significant differences between groups in the hypothallamus $(p<0.05)$, mesencephalon $(p<0.05)$, diencephallon $(p<0.01)$ and cerebellum $(p<0.05 \%$. Scheffe's t-test indicated that only in the hypothalamus and diencephalon consistent differences existed between TB and PF $(p<0.05)$ as well as between TB and FF $(p<0.01)$ groups (Table 6.3.-2).

In the MCA-tumor model ANOVA of the results of regional brain analysis showed significant TRP increments only in the diencephalon $(p<0.05)$ and corpus striatum $(0<0.05)$ of TB rats. In these animals, 5-HT was significantly elevated in corpus striatum $(p<0.01)$, diencephalon $(p<0.05)$, hippocampus $(p<0.05)$, pons/medulla $(p<0.01)$, cerebellum $(p<0.05)$ and cortex $(p<0.01)$, while 5-HIAA levels were significantly $(p<0.01)$ increased in all regions but the hypothaliamus (Fig. 6.3.-2).

The ratio of 5-HIIAA to $5-H T$, for each region also calculated in this tumor model, differed significantly by ANOVA between groups in the diencephalon $(p<0.001)$, mesencephalon $(p<0.004)$, cerebellum $(p<0.001)$, cortex $(\rho<0.001)$, corpus striatum $(p<0.001)$, pons/medulla ( $p<0.001)$ and hippocampus $(p<0.05)$.

Scheffé's t-test revealed significance between TB and PF groups as well as between TB and FF rats in all regions, but the hypothalamus (Table $6.3,-3$ ).

Table 6.3.-3 Mean ( \pm SEM) brain regional ratio of 5-HIAA to 5-HT in MCA tumor bearing (TB), and PF and FF control rats. At the bottom p values of post hoc t-tests.

\begin{tabular}{llll}
\hline Brain Area & $\begin{array}{l}\text { TB } \\
(\mathrm{n}=8)\end{array}$ & \multicolumn{1}{c}{$\begin{array}{l}\text { PF } \\
(\mathrm{n}=8)\end{array}$} & $\begin{array}{l}\mathrm{FF} \\
(\mathrm{n}=8)\end{array}$ \\
\hline Hypothalamus & $0.460 \pm 0.013$ & $0.431 \pm 0.018$ & $0.452 \pm 0.018$ \\
Corpus Striatum & $0.924 \pm 0.051$ & $0.641 \pm 0.032^{\mathrm{a}}$ & $0.658 \pm 0.018^{\mathrm{a}}$ \\
Mesencephalon & $0.992 \pm 0.057$ & $0.791 \pm 0.044^{\mathrm{c}}$ & $0.645 \pm 0.059^{\mathrm{a}}$ \\
Diencephalon & $0.745 \pm 0.028$ & $0.566 \pm 0.015^{\mathrm{b}}$ & $0.598 \pm 0.035^{\mathrm{b}}$ \\
Hippocampus & $0.818 \pm 0.042$ & $0.711 \pm 0.028^{\mathrm{c}}$ & $0.708 \pm 0.022^{\mathrm{c}}$ \\
Pons/Medulla & $0.858 \pm 0.035$ & $0.591 \pm 0.035^{\mathrm{a}}$ & $0.527 \pm 0.013^{\mathrm{a}}$ \\
Cerebellum & $1.081 \pm 0.078$ & $0.797 \pm 0.024^{\mathrm{b}}$ & $0.743 \pm 0.040^{\mathrm{a}}$ \\
Cortex & $0.576 \pm 0.024$ & $0.447 \pm 0.017^{\mathrm{a}}$ & $0.438 \pm 0.016^{\mathrm{a}}$
\end{tabular}

$a=p<0.001$ vs TB; $b=p<0.01$ vs TB; $c=p<0.05$ vs TB 


\subsection{Discussion}

Behavioral data in each of these experiments were similar to our earlier observations. Thus, elevated brain levels of TRP and indoles would support the original hypothesis of an involvement of $5-\mathrm{H} T$ in cancer anorexia.

Since we dissected a smaller hypothalamic area. TRP levels thus obtained for this region were presumably more representative of hypothalamic concentrations, since Knott (1974) described for this region a tendency towards a negative correlation between TRP concentrations and its weight. The assumption, that the observed values of TRP ${ }_{n} 5-H T$ and 5-HIAA in TB rats do indeed reflect effects induced by the presence of a tumar, is based on the good correlation between our control values for TRP, 5-HT and 5-HIAA and those reported in recent literature (Knott, 1974; Cummings, 1976). The brain TRP pattern in $W_{254}$ TB animals may very well reflect the increased transport into the brain of this amino acid. presumably resulting from its increased availability at the NAA-carrier site in the BBB, secondary to elevations of the unbound fraction of TRP in plasma. The changes in $5-H T$ levels, being limited to only two regions, may suggest regional differences in storage capacity (Knott, 1974). Changes in 5-HIAA levels were observed in diencephalon, hippocampus, pons/medulla, cerebellum and cortex. That these metabolite changes were more wide spread than for 5-HT, may be explained by regional differences of the $\mathrm{Km}$ of TRP-hydroxylase, as earlier suggested by Knott and Curzon (1974).

Neuronal indole activity, as suggested by the 5-HIAA/5-HT ratio, was increased to a greater extent in MCA TB rats, with all areas except the hypothalamus exhibiting significant elevations of this index as compared to increases in this ratio in $W_{256}$ TB rats only in the diencephalon and hypothalamus.

Also, the elevated 5-HT activity in the absence of generally increased TRP levels, may suggest an autonomous increase in 5-HT turnover in MCA TB animals. This observation is - if true - contrary to so far commonly accepted perceptions of the functioning of central indole amine systems, and needs further study.

The significance of the obserwed differences between the two tumor models is unclear, but may reflect the more prolonged anorexta in the MCA tumor model. Thus, elevated 5-HT activity within this tumor model appeared to involve all brain areas except the hypothalamus. Although the relationship of regional changes in 5-HT metabolism to feeding behavior is presently uncertain, it is apparent that 5-HT activity in the MCA tumor model is increased in areas rich in cell bodies (mesencephalon and pons/medulla) as well as in $5-\mathrm{HT}$ terminal areas (corpus striatum, diencephalon, hippocampus and cortex). The absence of changes within the hypothalamus is consistent with the prevalent hypothesis of cancer anorexia being mediated extrahypothalamically (Morrison, 1978). Thus, neither hyperphagia-producing lesions of the ventromedial hypothalamus (Baillie, 1965), nor lesions of the lateral hypothalamic areas (Baillie, 1965; Morrison, 1968) have any effect on the course of tumor induced anorexia. In addition, feeding elicited by exogenouslyadministered insulin. which is at least partially mediated by the hypothalamus (Epstein, 1967) appears to be intact in anorectic TB rats (Morrison, 1968).

Several areas of the limbic system, including the amygdala, olfactory cortex, hippocampus and septal area have been reported to influence food intake, as have been the mid brain tegmental, nigrostriatal and nucleus accumbens areas (Grossmann, 1980). Both MCA and $W_{256}$ TB animals exhibited elevated 5-HT activity in gross regions that included several of these areas 


\subsection{Conclusion}

Although the relationship of regional changes in 5-HT metabolism to feeding behavior is uncertain, these studies seem to indicate that 5-HT activity in anorectic TB rats is elevated in gross regions, that include areas that have been implicated biochemically and by lesion in the control of food intake. 


\section{Summary of the results and conclusions of the experiments described in part II}

\subsection{Tumor models}

\subsubsection{Walker 256 carcino sarcoma}

Intramuscular implantation of $5 \times 10^{4}$ viable $W_{256}$ tumor cells in immature female SpragueDawley rats resulted in spontaneous death of $80 \%$ of tumor bearing animals on day 10 post tumor implantation, and in significant anorexia in tumor bearing animals on day 6 , that increased in severity as the tumor grew. The tumor did not metastasize in this model other than to regional lymph nodes. Central necrosis was observed to a limited degree in the tumor and may be responsible for the increased white blood cell counts found in tumor bearing animals.

The significant loss of body weight in tumor bearing animals from the onset of anorexia as compared to freely feeding control animals was thought to originate from decreased food intake since it was paralleled by a simalar weight loss in pair-fed animals. Total body water and muscle water content were not different among groups, suggesting that the weight loss in tumor bearing and pair-fed animals occurred at the cost of lean tissue or fat compartments. Unaltered nitrogen retention in tumor bearing and pair-fed groups as compared to freely feeding control animals, in combination with unchanged urinary 3-methy/histidine excretion, as a parameter for muscle wasting and unaltered dry muscle weights, suggested however that loss of lean body tissue did not contribute to the observed body weight loss. On the other hand, both unaltered nitrogen retention and urinary 3-methylhistidine excretion in tumor bearing animals do not indicate that no nitrogen translocation between host and tumor occurred. Plasma albumin levels being decreased in tumor bearing animals, presumably as a result of deposition in the tumor, may serve as illustration to the above.

The higher dry weights of liver tissue in tumor bearing animals as compared to these values in the comparably starved pair-fed animals, may result from increased protein synthesis activity. It is of considerable interest that this change occurred first rellative to the onset of cancer anorexia.

It was concluded that the $W_{256}$ tumor in immature female Sprague-Dawley rats is a highly reproducible model of cancer anorexia, that is so acute in nature that only mild cachexia will develop before the tumor kills the host.

\subsubsection{Methylcholanthrene induced sarcoma}

Subcutaneous implantation of $2 \times 10^{5}$ viable methylcholanthrene sarcoma cells in adult male Fisher 344 rats resulted in prolonged anorexia and spontaneous death of the tumor bearing animals. The onset of anorexia and the time of spontaneous death varied to such a degree, that no reproducible moment of anorexia across experiments was observed. Net body weights in tumor bearing animals were less than those of pair-fed and freely feeding control animals, while food intake of tumor bearing animals was insufficient to support normal 
growth in pair-fed animals. Analysis of muscle dry weights and water soluble protein contents revealed a considerable loss of lean body tissue in tumor bearing animals. Dry weight and total water soluble protein content of liver tissue were decreased in tumor bearing and pair-fed rats as compared to freely feeding controls. Relative to the more pronounced weight loss these values were increased in tumor bearing animals as compared to pair-fed rats. This again, may be a result from increased protein synthesis.

It was concluded that the methylcholanthrene induced sarcoma in adult male Fisher 344 rats is a model of prolonged anorexia and severe cancer cachexia, ultimately resulting in death of the lumor host.

\subsection{Serotonin}

\subsubsection{Plasma tryptophan}

Plasma albumin concentrations were decreased in tumor bearing animals of both tumor lines, resulting in decreased binding capacity in plasma for tryptophan. Together with presumed increased plasma levels of free fatty acids this observation was considered to cause the observed elevation of plasma free tryptophan levels in tumor bearing rats, even though plasma total tryptophan levels were decreased in these animals, which is presumably a result of decreased food intake.

\subsubsection{Blood-brain barrier transport}

Of those factors important for tryptophan transport into the brain, plasma free tryptophan was elevated in both tumor models. Because the sum of the neutral amino acids, that compete for the same blood-brain barrier amino acid carrier, was not changed, the changes observed so far would result in competitive advantage of tryptophan over the other neutral amino acids for transport into the brain. This observation, expressed in the ratio plasma free tryptophan over the other neutral amino acids, was made in the $W_{256}$ tumor model.

The activity of the blood-brain barrier neutral amino acid transport system was assessed by determination of the brain uptake index for tryptophan, and found to be unchanged. This observaton was only made in the $W_{256}$ tumor model.

\subsubsection{Brain tryptophan serotonin and 5-hydroxyindoleacetic acid}

Brain tryptophan values were, as expected from the above observations, elevated in brains of tumor bearing rats of both tumor lines. It was assumed, that as a result of the increased precursor availability serotonin concentrations were elevated. In addition, serotonin degradation was increased as illustrated by increased 5-HIAA concentrations.

These alterations in the serotonin system, present in two tumor lines, thus appeared to be a general phenomenon. Additionally, the absence of such changes in the significantly malnourished pair-fed animals in the methylchollanthrene experiments suggests that they are causative in rather than a consequence of the development of cancer anorexia. This concept seems to be supported by results that indicate increased serotomergic activity being present just prior to the onset of anorexia.

Although the relationship of regional changes in serotonin metabolism to feeding behavior is uncertain, results of regional serotonin determinations in both tumor models seem to indicate that serotonergic activity in anorectic tumor bearing rats is elevated in gross regions, that include areas that have been implicated biochemically and by lesion in the control of food intake. 


\subsubsection{Conclusion}

Merely by association of the above summarized observations of increased central serotonergic activity within both an acute and a chronic model of cancer anorexia, arising just prior to the onset of anorexia, and of regional serotonin differences in areas implicated in the control of food intake, with the published claims of serotonin mediating satiety, the theory is formulated that cancer anorexia may be mediated, at least in part, by serotonin.

\subsection{Catecholamines}

Contradictory results regarding catecholaminergic systems were obtained. Thus, no consistent pattern of change in brain catecholamine concentrations correlated with the onset or severity of cancer anorexia was observed in the $W_{256}$ tumor model, while in MCA tumor bearing animals no decreased amines in comparison with pair-fed rats were observed. The latter finding may suggest a role for adrenergic systems in the genesis of cancer anorexia by falsely signalling satiety or failling to adequately signal hunger in tumor bearing animalls.

It should be concluded that a more systematic inwestigation into the role of catecholamines in the cause of cancer anorexia must be performed. 
Part III

Efforts to manipulate the entry of tryptophan into the brain and the indoleamine system

in cancer anorexia 


\section{Chapter 8}

\section{The effect of branched-chain amino acids enriched diets on food intake of tumor bearing animals}

\subsection{Introduction}

Tryptophan and the other neutral amino acids (NAA) share a common carrier system for transport across the blood-brain barrier (BBB) (Oldendorf, 1971). The $\mathrm{Km}$ of this $\mathrm{BBB}$ amino acid carrier approximates the plasma amino acid concentration of these NAA. This close relationship provides the basis for the marked sensitivity of the brain to amino acid competition (McMenamy, 1961; Pardridge, 1979atb). Thus, increasing plasma branched chain amino acid (BCAA) levels would presumably result in increased competition with TRP for binding to the BBB carrier, yielding in the presence of normal carrier activity (Krause, $1979^{\mathrm{a}}$; chapter 3 this thesis) lower brain TRP levels. Consequently serotonin synthesis would be reduced by decreased precursor availability (Fernstrom, 1977; Pardridge, 1979 ${ }^{\mathrm{b}}$ ). We therefore designed an experiment exploring the effects of BCAA enriched diets on food intake of TB and FF control animals, as compared to the effects on ingestive behavior of an isonitrogenous casein control diet.

\subsection{Materials and methods}

\section{Design}

Sixty two immature female SD rats served as subjects across two experiments. In experiment 1,30 rats were divided into four groups; TB-BCAA $\mid(n=12)$, TB-Casein I $(n=6)$, FF-BCAA I $(n=8)$ and FF-Casein $\|(n=4)$. TB animals were inoculated im. with $5 \times 10^{4}$ $W_{256}$ tumor cells on day 0 , while control animals received an equal volume norma! saline. All rats had free access to the test or control diets and water. Beside routine daily monitoring, $24 \mathrm{hr}$ urine production was collected on $3 \mathrm{cc} 12 \mathrm{~N} \mathrm{HCl}$. After recording volumes of urine, an aliquot of each portion was frozen and stored at $-70^{\circ} \mathrm{C}$ for later determination of nitrogen content. All animals were sacrificed on day 9 post tumor implantation. In experiment 2,32 animals were divicled over 4 groups: TB-BCAA II $(n=8)$, TB-Casein II $(n=8)$. FF-BCAA $\|(n=8)$ and FF-Cas $\|(n=8)$. Again, TB animals were inoculated with the $W_{256}$ tumor, while controls received a sham injection normal saline. With either test or controll diets freely feeding animals were monitored for daily food intake and body weight changes. After 9 days of tumor growth all animals were sacrificed by decapitation.

\section{Diets}

A pelleted standard casein laboratory rat chow, containing $25 \%$ branched chain amino acids and $1,2 \%$ TRP of total casein (ICN Nutritional Biochemicals Cleveland, Ohio), was enriched with $\mathrm{BCAA}$ or vitamin free casein to obtain a BCAA enriched test diet and an isonitrogenous casein control diet. Thus, by roughly doubling the original amounts of each one of the BCAA in casein, the test diet used in experiment 1 , BCAA-1, turned out to contain $37 \%$ BCAA of total casein. The second test diet, BCAA- 11 was found to contain $47 \%$ BCAA of total casein by tripling the original amounts of these AA in casein. Total nitrogen content of the first test and control diets was $37.5 \mathrm{mg} / \mathrm{g}$ diet, while the second diet pair contained $39 \mathrm{mg}$ nitrogen per gram diet. 


\section{Dissection}

At sacrifice in experiment 1, blood was collected tor determination of plasma total and free TRP, and amino acids.

From all animals sacrificed in experiment 2 blood and brains were harvested for determination of plasma total and free TRP, amino acids and brain TRP, 5-HT and 5-HIAA.

Biochemical analysis

Plasma amino acids, total and free TAP, and brain TRP, 5-HT and 5-HIAA were determined according to techniques described in chapter 2.

Urinary nitrogen content was determined according to the technique described in chapter 3 . section 3.2

\subsection{Results}

Mean food intake in the first experiment is shown in fig. 8.3.-1. Statistical evaluation, employing a repeated measures analysis of variance over days 5 through 9 , indlicated significant differences in tood intake between TB and FF groups $(F(1.26)=36.44: p<0.001)$, between the diets $(F(1.26)=4.35: p<0.05)$ and across time $(F(4,104)=330.88 ; p<0.001)$. Also, there were significant interactions between time and tumor $(F(4,104)=74.63 ; p<0.001)$, time and diets $(F(4.104)=74.75 ; p=0.001)$ and time, tumor and diets $(F(4,104)=348.53 ; p<0.001)$, suggesting that the onset of anorexia in TB rats, fed the $37 \%$ BCAA diet, was significantly delayed. Post hoc t-tests indicalted no differences between FF-BCAA I and FF-Cas I groups on any days. The TB-Cas I group ate significantly less than both FF groups an days $6 .(P<0.05), 7(0<0.001)$, 8 and $9(\rho<0,01)$. The TB-BCAA I group however, did not differ from FF groups on days 6 and

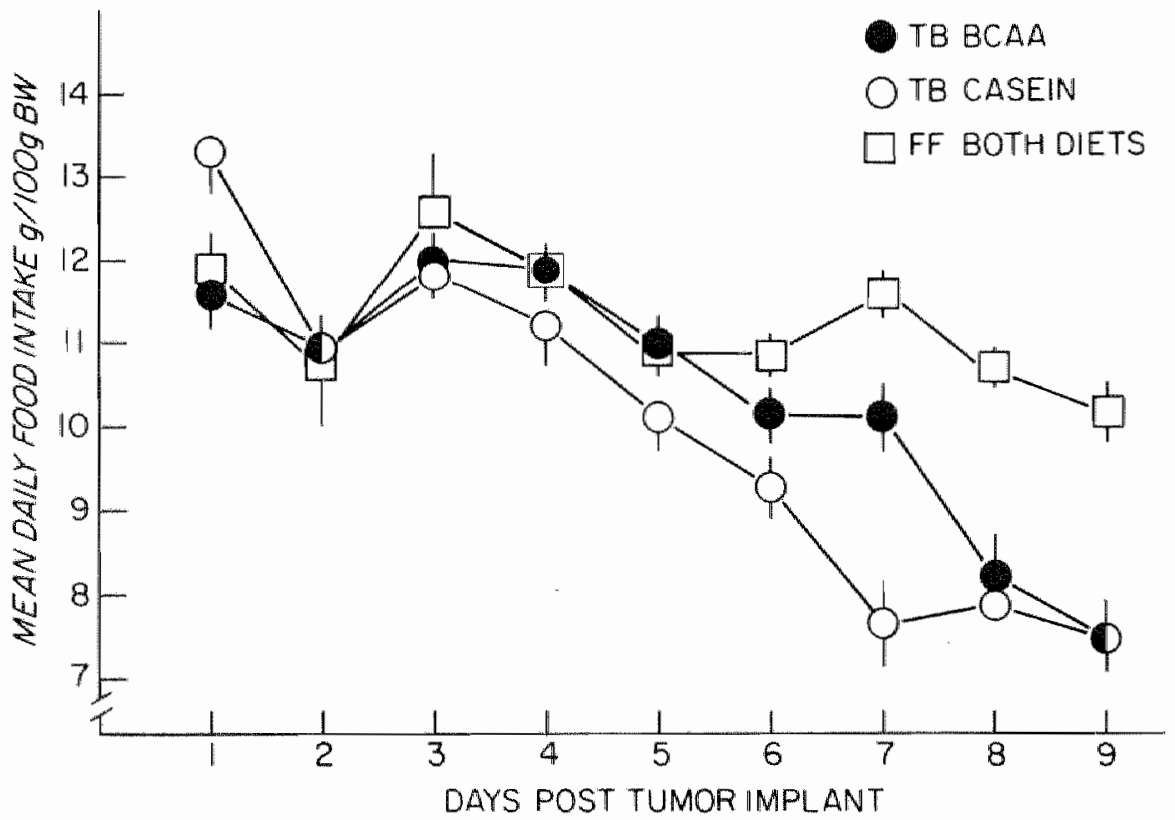

Fig. 8.3.-1 Mean (1 SEM) daily ad lib. food intake by TB ( $W_{256}$ tumor, circles) and FF control rats (squares), maintained on a $37 \%$ BCAA enriched diet (closed symbols) or an isonitrogenous casein contral diet (open symbols). 
7 , but displayed significant anorexia on days 8 and $9(0<0.01)$. In addition, the TB-BCAA I group consumed significantly $(0<0.01)$ more food than the TB-Cas $/$ animals did on day 7 . Therefore, it is suggested that significant anorexia may have been delayed by 2 days by providing a BCAA enriched diet to TB animals.

Analysis of biochemical data from experiment 1 by a wo-way ANOVA (Table 8.3.-1.) indicated significant elevations of plasma free TRP $(F(1,26)=120.06: 00.00 \%)$ while plasma total TRP levels were decreased $(F(1,26)=47.52 ; p<0.001)$ in TB rats. Changes in the FF groups for total TRP amounted to a significant diet effect $(F(1,26)=8.87: p<0.01)$, while significant tumor $x$ diet interactions were observed for plasma free TRP $(F(1.26)=8.51 ; 0<0.01)$ and plasma total TRP $(F(1.26)=23.52: p<0.001)$. Post hoc t-tests revealed that plasma TRP levels in TB animals were not affected by the diet, but total TRP values were significantly $(0<0.05)$ lower in FF-BCAA I animals as compared to FF-Cas I animals.

Significant diet effects were observed in the plasma levels of Valine $(F(1,26)=4.99, p<0.05)$. Isoleucine $(F(1,26)=5.00 ; p<0.05)$ and Leucine $(F(1,26)=6.72 ; p<0.05)$. These alterations were, however, insufficient to induce significant diet effects in the ratio plasma free TRP over the sum of neutral $A A$, that was significantly $(F(1,26)=42.31: P<0.001)$ elevated in TB-animals (Table 8.3.-1).

Mean cumulative nitrogen retention was significantly $(F(1,26)=11.67 ; p<0.01)$ reduced in $T B$ animals but significantly $(F(1,26)=70.21 ; p<0.001)$ increased under influence of the BCAA-diet (Fig. 8.3.-2).

In an effort to obtain a further delay in the onset of cancer anorexia, the experiment was repeated with the test diet containing higher BCAA concentrations. Mean food intake data from this experiment are depicted in Fig. 8.3.-3. Statistical analysis, again by a repeated measures ANOVA over days 5 through 9 , indicated significant differences in food intake between TB and $F F$ groups $(F(1,31)=35.80: p<0.001)$, between the diets $(F(1,31)=21.52 ; p<0.001)$ and actross time $(F(1,128)=37.40, p<0.001)$. Again there were significant interactions between time and tumor $(F(4,128)=21.10 ; p<0.001)$ and time and diets $(F(4,128)=6.87 ; p<0.001)$, but not between time, tumor and diets, indicating that the $47 \%$ BCAA diet did not differentially influence TB animals, as far as the onset of anorexia was concerned (Fig. 8.3.-3).

Table 8.3.-1 Mean ( \pm SEM) plasma levels of free and total TRP, valine, leucine, isoleucine and the ratio plasma free TRP/ENAA of tumor bearing and control animals, fed a 37\% BCAA or control diet, obtained at sacrifice 9 days after the inoculation of $W_{256}$ tumors. At the bottom $p$ values of post hoc t- tests.

\begin{tabular}{|c|c|c|c|c|c|c|}
\hline & $\begin{array}{l}\text { Free TRP } \\
(\text { mimol/mil) }\end{array}$ & $\begin{array}{l}\text { Total TRP } \\
(\mathrm{n} m \mathrm{~mol} / \mathrm{ml})\end{array}$ & $\begin{array}{l}\text { Valine } \\
\text { (nmol/ml) }\end{array}$ & $\begin{array}{l}\text { Leucine } \\
(\mathrm{nmol} / \mathrm{ml})\end{array}$ & $\begin{array}{l}\text { Isoleucine } \\
\text { (nmal/ml) }\end{array}$ & $\frac{\text { Free TAP }}{2 N A A}$ \\
\hline $\begin{array}{l}\text { TB-BCAA } \\
(17=12)\end{array}$ & $21.6 \pm 1.0$ & $49.4 \pm 3.5$ & $318.0 \pm 44.3^{b}$ & $213.4 \pm 30.2^{b}$ & $126.2 \pm 16.6^{b}$ & $0.0308 \pm 0.0037$ \\
\hline $\begin{array}{l}\text { TB-Casein I } \\
(n=6)\end{array}$ & $23.1 \pm 1.4$ & $46.6 \pm 2.7$ & $193.5 \pm 8.3$ & $113.5 \pm 3.0$ & $83.2 \pm 3.7$ & $0.0393 \pm 0.0022$ \\
\hline $\begin{array}{l}\text { FF-BCAA I } \\
(n=8)\end{array}$ & $14.4 \pm 11.4^{a}$ & $58.9 \pm 3.1^{a b}$ & $311.5 \pm 61.3^{b}$ & $205.9 \pm 37.6^{b}$ & $121.8 \pm 18.9^{b}$ & $0.0222 \pm 0.0035^{3}$ \\
\hline $\begin{array}{l}\text { FF-Casein! } \\
(n=4)\end{array}$ & $10.9 \pm 1.0^{a}$ & $84.3 \pm 9.6^{3}$ & $206.3 \pm 11.8$ & $135.3 \pm 9.1$ & $85.0 \pm 3.7$ & $0.0173 \pm 0.0021$ \\
\hline
\end{tabular}

$a=p<0.01$ ws TB, same diet; $b=p<0.05$ vs controll diet 


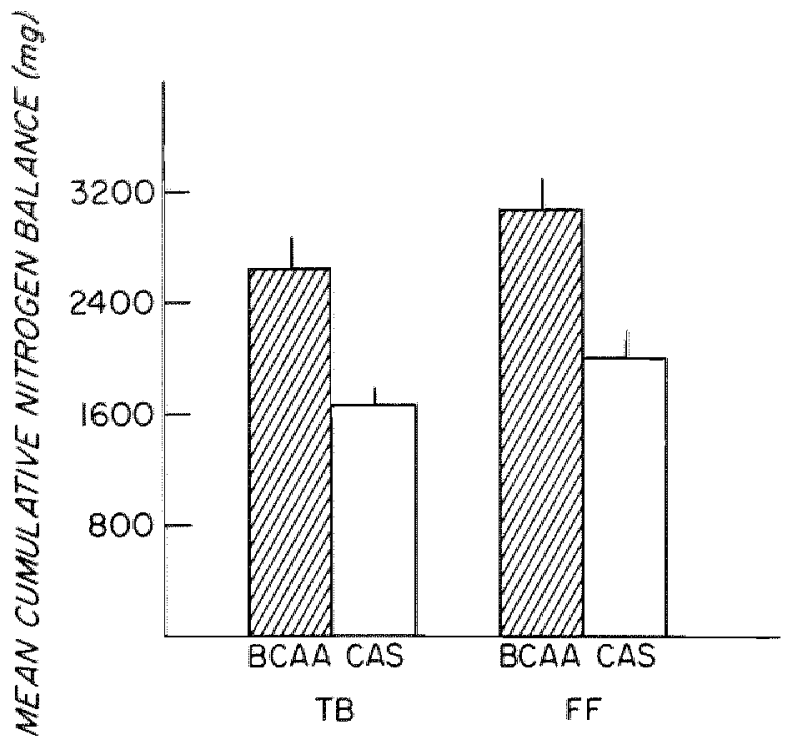

Fig. 8.3,-2 Mean (1 SEM) cumulative nitrogen balance (mg) 9 days after im. Walker 256 tumor implantation (TB, left bars) or sham injection normal saline (FF, right bars) in immature SD rais, fed a $37 \%$ BCAA (hatched bars) or control diet (open bars).

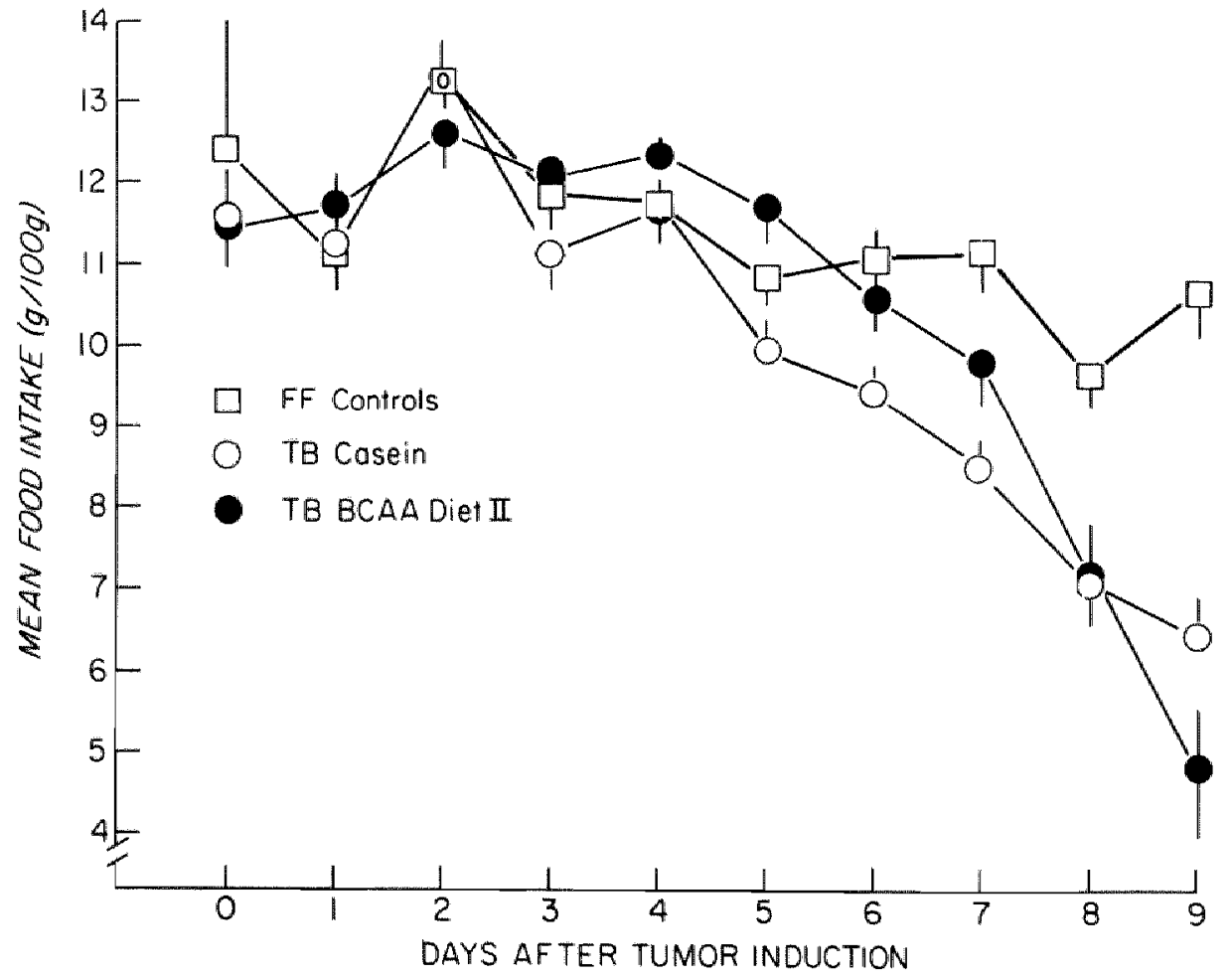

Fig. 8.3.*3 Mean (1 SEM) dailly ad lib, food intake by TB (Walker 256 tumor, circles) and FF control rats (squares) maintained on a highly enriched BCAA diet (47\% af casein. ciosed symbols) or an isonitrogenous casein control diet (open symbols). 


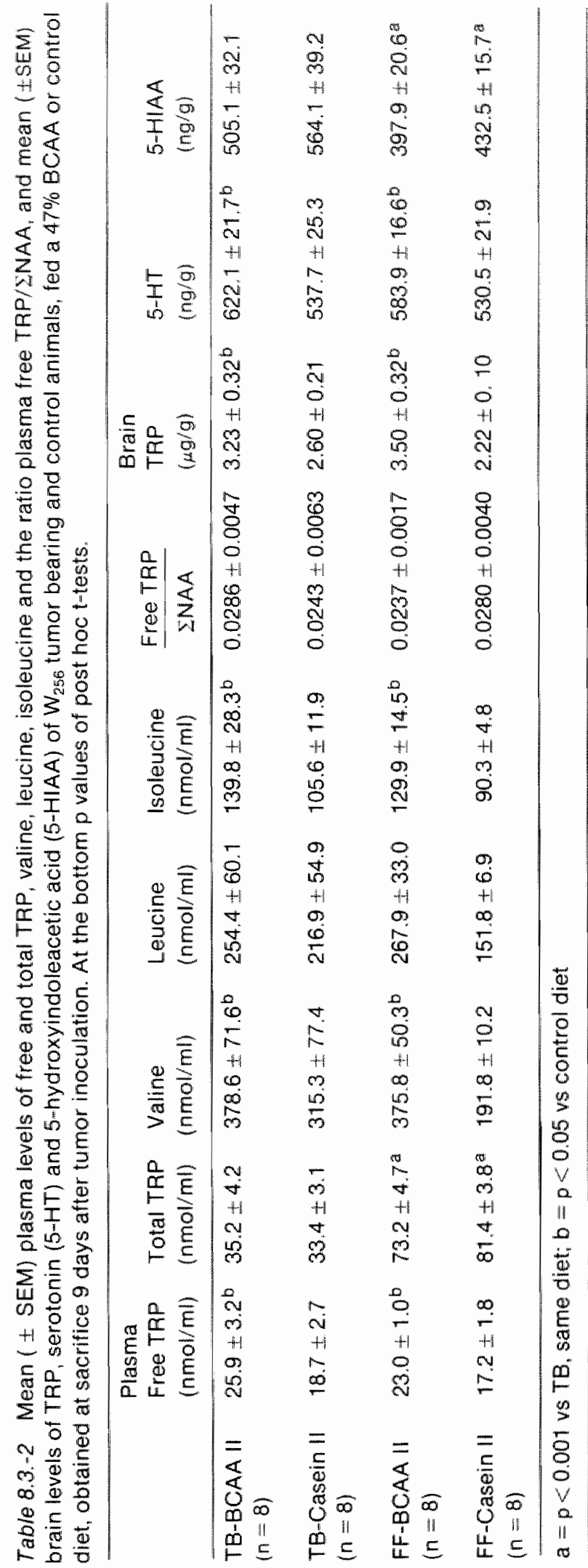


Table 8.3.-3 Plasma albumin values in Walker 256 TB animals, expressed as percent of values in $\mathrm{FF}$ animals, obtained at sacrifice. 9 days after tumor inoculation.

\begin{tabular}{lllll}
\hline & $\begin{array}{l}\text { Chow } \\
(17 \% \text { BCAA })\end{array}$ & $\begin{array}{l}\text { Casein } \\
(25 \% \text { BCAA })\end{array}$ & $\begin{array}{l}\text { BCAA 1-diet } \\
(37 \% \text { BCAA })\end{array}$ & $\begin{array}{l}\text { BCAA ll-diet } \\
(47 \% \text { BCAA })\end{array}$ \\
\hline Albumin & $67 \%$ & $71 \%$ & $89 \%$ & $94 \%$ \\
\hline
\end{tabular}

Analysis of biochemical data from this - second - experiment, again by a two-way ANOVA, (table 8.3.-2), indicated a significant diet effect $(F(1,28)=7.55: p<0.01)$ for free TRP values, while total TRP was significantly decreased $(F(1,28)=106.63 ; p<0.001)$ in TB animals. Post hoc $t$ tests indicated significantly increased free TRP levels $(\rho<0.05)$ in animals supplied with the $47 \%$ BCAA diet, while TB groups showed significantly $(p<0.001)$ decreased plasma total TRP values across both diets. Significant diet effects were observed only for Valine $(F(1,28)=4.81$ $p<0.05)$ and isoleucine $(F(1,28)=4.48 ; p<0.05)$. Due to trends towards increased levels of $B C A A$ in TB animals, that was most apparent in Leucine, no diet effect was observed for this amino acid.

Brain TRP levels showed a significant diet effect $(F(1,28)=13.90 ; p<0.001)$, thereby following plasma tree TRP walues. Brain 5-HT levels were also diet affected $(F(1,28)=10.31: p<0.01)$. 5 -HIAA values however showed a significant $(F(1,28)=18.04 ; p<0.001)$ tumor effect. Post hoc t-tests indicated significant $(p<0.05)$ elevations of brain TRP and 5-HT in BCAA groups, compared to casein groups, while brain 5-HIAA levels were significantlly $(0<0,001)$ elevated in TB groups.

Inter-assay variability of albumin determinations required that albumin values be expressed as percent of values in FF animals (table 8.3.-3). Results show a high correlation $(r=0.9783$ ) between percentage BCAA content of the diet, and the albumin values.

\subsection{Discussion}

It is not certain, that the behavioral changes demonstrated in experiment 1 , are a result of increased competition with TRP at the BBB neutral amino acid carrier site induced by the diet enrichment with BCAA. Thus, although plasma levels of the branched chain amino acids were significantly increased under influence of the BCAA enriched diet no effect was observed in the ratio plasma free TRP over the sum of NAA. It must be assumed, that the diet effect on BCAA levels and on free TRP levels neutralized each other, resulting in the absence of any change in the ratio free TRP over the sum of neutral amino acids. This ratio, however, correlated well with behavioral data: the groups with elevated ratios were anorectic.

The lack of effect of the diet on the ratio in FF-BCAA rats, and the results of the nitrogen balance studies make one speculate about the way the BCAA exerted their effects. The $\mathrm{BCAA}$ are the only amino acids that are principally oxidized by skeletal muscle (Odessey, 1974; Buse, 1975). The rate of oxidation in muscle is stimulated by fasting, stress, diabetes and conditions that are usually associated with protein wasting and nitrogen loss. Oxidation of BCAA supplies energy to the musclle and nitrogen for the glucose-alanine cyle and muscle glutamine synthesis (Felig, 1973; Odessey, 1974). Furthermore, recent reports (Odessey, 1974; Buse, 1975; Fulks, 1975; Freund, 1981) suggest a regulatory role for these amino acids, by promoting protein synthesis, and reducing protein degradation. These properties were used by Blackburn (1979) to explain enhanced nitrogen retention and increased protein synthesis in muscle and liver in septic traumatized rats. In addition, the same group has recently (Kawamura, 1982) suggested improved protein utilization and 
reduced cachexia in tumor bearing rats, infused with a $25 \%$ BCAA solution. We therefore tend to believe that the behavioral effects in experiment 1 resulted from a better overall condition of TB animals, postponing lipolysis, and maintaining albumin levels (Kirsch, 1969). thus keeping plasma free TRP Iow, rather than from BBB competition effects. The disappearance of a significant differential effect of the $47 \%$ BCAA diet on food intake in the second experiment may be the result of several factors. The BCAA enrichment may have induced a diet imbalance resulting in reduced food intake (Rogers, 1973). Also, the increased nitrogen content of both test and control diets may exert an inhibitory effect to such an extent (Rogers, 1973), that a possibly facilitory effect of the BCAA addition is overshadowed. Finally, learned food aversions may play a role (Bernstein, 1980).

The cause of the trend of plasma total TRP to be lower in FF-BCAA animals is uncertain. th cannot be explained by casein enrichment of the diet inducing relatively increased TRP intake in casein control diet animals, since casein contains only $1.2 \%$ TRP. Neither can increased degradation of TRP explain the effect, since the rate limiting enzymatic step in this proces is known to be induced only by adrenal corticosteroids and TRP itself (Rodwell. 1977). Enhanced protein synthesis under influence of BCAA (Buse, 1975; Fulks, 1975;

Freund, 1981) in these growing animals however may account for the lowering of plasma total TRP, and for this trend being observed only in FF animals.

The trend of plasma free TRP to increase in FF animals consuming the $37 \%$ BCAA diet, and this trend becoming significant in both TB and FF animals consuming the $47 \%$ BCAA diet is unexplicable without further research.

In conclusion, we tend to believe that the addition of BCAA to a diet would maintain/induce high plasma albumin levels, and limit lipolysis, thus decreasing plasma free fatty acid levels. Thus, these effects would postpone the elevation of plasma free TRP levels.

\subsection{Conclusions}

1. A $37 \%$ BCAA diet causes a 48 -hour delay in the onset of cancer anorexia in $W_{256}$ tumor bearing rats. This effect does not seem to be dose-dependent, as further enrichment with $B C A A$ does not induce a further delay in the onset of anorexia.

2. The mechanism of this BCAA effect is not known, but may be a result of enhanced protein conservation in the TB host, rather than increased competition at the BBB carrier site, as was predicted.

3. BCAA may induce protein synthesis in FF animals to such a degree that TRP incorporation in protein results in decreased plasma total TAP concentrations.

4. No explanation is available for the increments of plasma free TRP under influence of the consumption of a BCAA enriched diet. 


\section{Chapter 9}

\section{The effect of parachlorophenylalanine on serotonin synthesis and food intake in cancer anorexia}

\subsection{Introduction}

Parachlorophenylalanine ( $p-C P A)$ is known to deplete brain serotonin selectively by inhibition of tryptophan hydroxylase (Koe, 1966, Jequier, 1967). This drug also reduces brain CA for the limited period of 2 days. The effect of intraventricular (ivt.) p-CPA administration on food intake was investigated by Breisch (1976). They reported overeating in rats 3 days after administration of the drug, lasting for 1 to 2 weeks, and leading to increased body weights. These behavioral changes took place in the presence of forebrain serotonin depletions varying from 29 to 78 percent. Thus, central serotonin depletion in TB rats by ivt. administration of this TRP hydroxylase inhibitor would presumably result in a delay or complete remission of cancer induced anorexia if serotonin mediated this phenomenon. We therefore employed the acute ivt. administration of p-CPA in the following experiments.

\subsection{Materials and methods}

\section{Design}

Fifty nine immature $(80-100 \mathrm{~g})$ female SD rats served as subjects in two experiments and were treated and monitored in the standard fashion.

In experiment 1, thirty rats were divided into four groups: TB-p-CPA $(n=7)$, TB-Sal $(n=8)$, FF-p-CPA ( $n=7)$ and FF-Sal $(n=8)$. To coincide the maximum effect of $p$-CPA with the onset of anorexia, the drug was injected 2 days after the im. injection of $5 \times 10^{d} W_{256}$ tumor cells or normal saline. All rats were sacrificed by decapitation 9 days after tumor induction.

Because of untimely death of one TB-Sal animal, biochemical analyses could only be performed on 7 animals in this group.

In experiment 2, twenty nine animals were divided over four groups: TB-p-CPA $(n=8)$, TB-Sal $(n=7)$, FF-p-CPA $(n=7)$ and FF-Sal $(n=7)$. Agal $n$, TB animals were inoculated im. with $W_{256}$ tumor cells, while controls received a sham injection normal saline. p-CPA was, as in

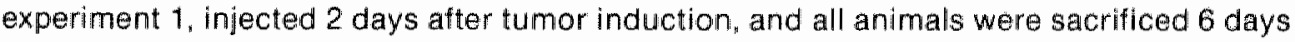
post tumor implantation.

\section{Intraventricular injection of $p-C P A$}

Eight $\mu$ l of normal saline containing $2 \mathrm{mg}$ of D.L.-p-CPA methylester was bilaterally injected (ivt.) under ether anesthesia in 15 of the TB and 14 of the non tumor bearing (FF) animals. The drug was purchased from Sigma Chemical Company (St. Louis, MO) and the given dose corresponded with the dose Breisch (1976) used. The other 15 animals in each group received sham injections (ivt.) with an equal volume of normal saline at the same time. Each injection was delivered over a 4 minute time period through a 31 ga hypodermic needle connected to a $50 \mu$ syringe (Hamilton Company, Reno. Nev.). The hypodermic needle was stereotaxically positioned at the following coordinates taken from bregma: $A-0.5, L \pm 1.5$ and $V-3.0 \mathrm{~mm}$ (Pellegrino and Cushman Atlas). Following their surgery rats were returned to their home cages, where standard monitoring continued. 


\section{Biochemical analysis}

Following sacrifice by decapitation brains were removed hemisectioned and frozen in liquid nitrogen for determination of brain TRP, 5-HT and 5-HIAA, whille blood was collected for assay of plasma free and total TRP. In addition to measuring indole activity, norepinephrine (NE) and dopamine (DA) were assayed in experiment 2.

\subsection{Results}

Mean food intake in the first experiment is presented in Fig. 9.3-1. As may be observed, food intake was decreased under influence of the operative procedure. Further more, the onset of anorexia in TB rats following the ivt. injections of p-CPA was delayed. Statistical analysis employing a repeated measures analysis of variance over days 9 through 13 , indicated significarit drug $(F(1,26)=11.53 ; p<0.01)$, tumor $(F(1,26)=24.78 ; p<0.001)$ and time $(F(4,104)=52.26 ; p<0.001)$ effects. The significant $(p<0.01)$ interaction effects (time $x$ drug. time $x$ tumor and time $x$ tumor $x$ drug) however preclude a simple interpretation of the data. Post hoc $t$-tests showed no difference between iwt. saline (Sal) injected FF and FF-p-CPA groups on any days. The TB-Sal group however, consumed significantly $(p<0.01)$ less food than did the FF-Sal group on days 10,11, 12 and 13. Although the TB-p-CPA group did not differ from the FF-p-CPA group on days 9 and 10 , significant $(p<0.05)$ changes were observed on days 11,12 and 13. In addition, the TB-p-CPA group ate significantly more on days 9 $(p<0.01), 10(p<0.05)$ and $11(p<0.05)$ than did the TB-Sal group. Therefore, it appears that significant anorexia may have been delayed by 2 days (until day 12 ) following the ivt. injection of p-CPA. Figure 9.3.-2 presents mean water intake by the various groups of rats in

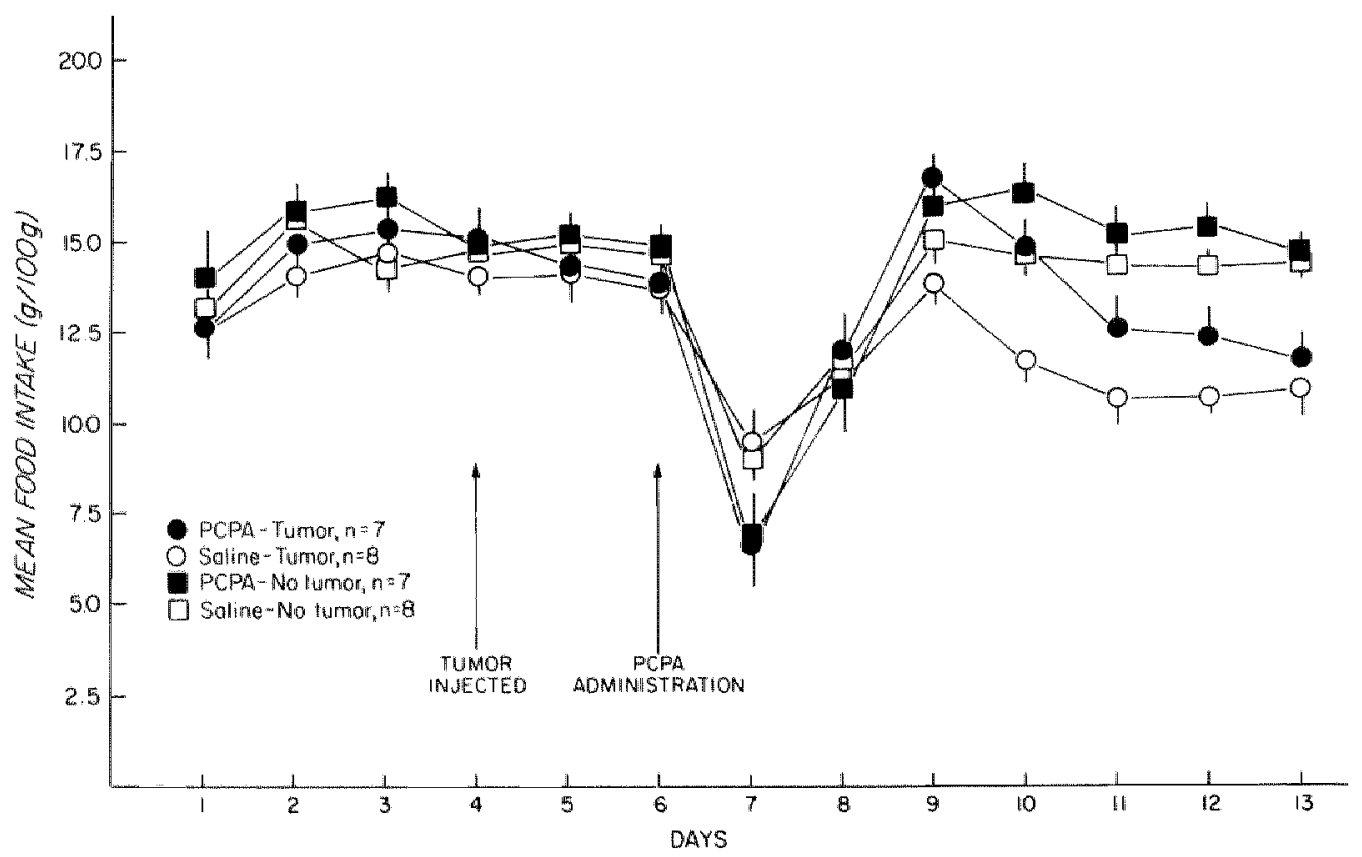

Fig. 9.3.-1 Meary (t SEM) dailly food intake of tumor bearing (circles) and control (squares) rats following the bilateral intraventricular injection $(8 \mu 1)$ of $2 \mathrm{mg}$ of $\mathrm{DL}-\mathrm{parachlorophenylalanime} \mathrm{(D-CPA)}$ methylester (closed symbols) or normal saline (open symbols). Alll rats were sacrificed 9 days after the injection (im.) of $5 \times 10^{4}$ Walker 256 carcino sarcioma cells or normal saline (Exp. 1\}. 


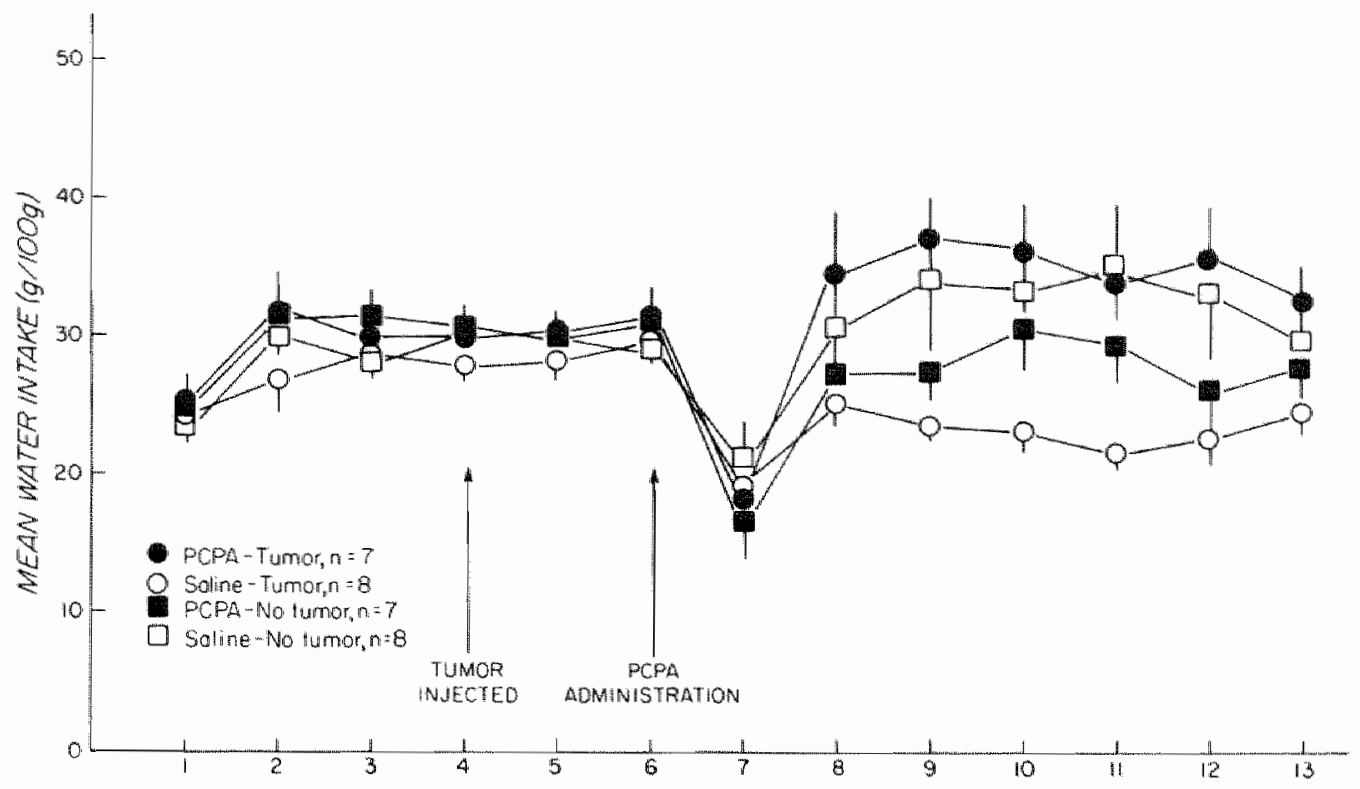

DAYS

Fig. 9.3.-2 Mean ( \pm SEM) daily water intake of tumor bearing (circles) and control (squares) rats following the bilateral intraventricular injection $(8 \mu$ ) of $2 \mathrm{mg}$ DL-p-CPA methylester (closed symbols) or normal saline (open symbols). All rats were sacrificed 9 days after the injection (im.) of $5 \times 10^{4}$ $W_{256}$ tumor cells or normal saline (Exp. 1).

this experiment. Although water intake roughly followed food consumption, all groups of rats continued to drink adequate amounts. Thus, even the TB-Sal rats continued to consume over $20 \mathrm{~g} / 100 \mathrm{~g}$ body weight throughout the anorectic period.

Analysis of the biochemical data for experiment 1 (Table 9.3.-1) indicated that although plasma total TRP was decreased $(F\{1,25)=17.59 ; p<0.001 ; 2$-way ANOVA), plasma free TRP was elevated $(F(1,25)=28.58 ; p<0.001)$ in TB-rats. Plasma TRP was not affected by the $p$-CPA treatment in either TB or FF rats. Brain TRP was also elevated in TB rats $(F(1.25)=10.24$; $p<0.01)$ as was brain $5-\mathrm{HIAA}(F(1,25)=13.08 ; p<0.01)$. However, $\mathrm{p}$-CPA treatment significantly reduced both brain $5-H T(F(1,25)=11.49 ; p<0.01\})$ and $5-H \mid A A(F(1,25)-15.21 ; p<0.001)$, with post hoc t-tests revealing decreased $5-H T$ in FF $(p<0.05)$ and decreased $5-H I A A$ in FF $(p-0.05)$ and TB $(p<0.01)$ groups.

In order to obtain biochemical data that more closely corresponded to the onset of the anti-anorectic effect of $p-C P A$, the experiment was repeated with the rats being sacrificed 4 days after the ivt. injection. As may be observed in fig. 9.3.-4, the effect of $p$-CPA on food intake was similar to the results of the previous experiment. Statistical analysis of food intake data across days 8 through 10 (repeated measures ANOVA) revealed a significant overall effect of $p-C P A(F(1,25)=6.14 ; p<0.05)$ and a significant time $x$ fumor interaction $(F(2,48)=6.54, p<0.01)$. Post hoc $t$-tests indicated the TB-Sal rats to be eating less than the FF group by day $9(p<0.05)$ and the TB-p-CPA group to be eating more than the TB-Sal group on day $10(p<0.01)$.

Analysis of the biochemical data from this experiment (Table 9.3.-2) suggests that $p-C P A$ significantly reduced $5-H T(F(1,25)=14.58 ; p<0.001)$ as well as $5-H I A A(F(1,25)=13.59 ; p<0.01)$. 


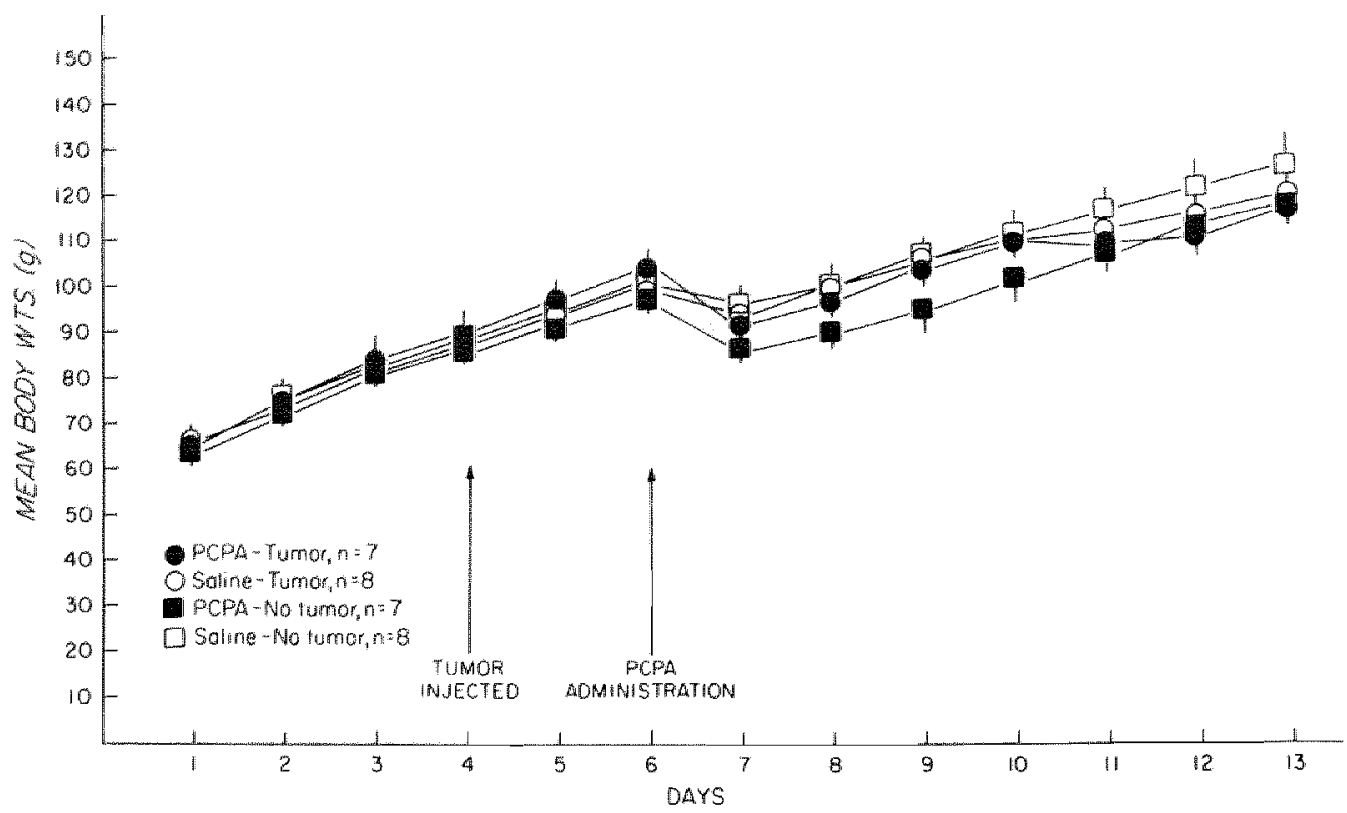

Fig. 9.3.-3' Mean (t SEM) body weights of tumor bearing (circles) and control (squares) rats following bilateral ivt. administration of DL-p-CPA (closed symbols) or normal saline (open symbols) in Exp. 1.

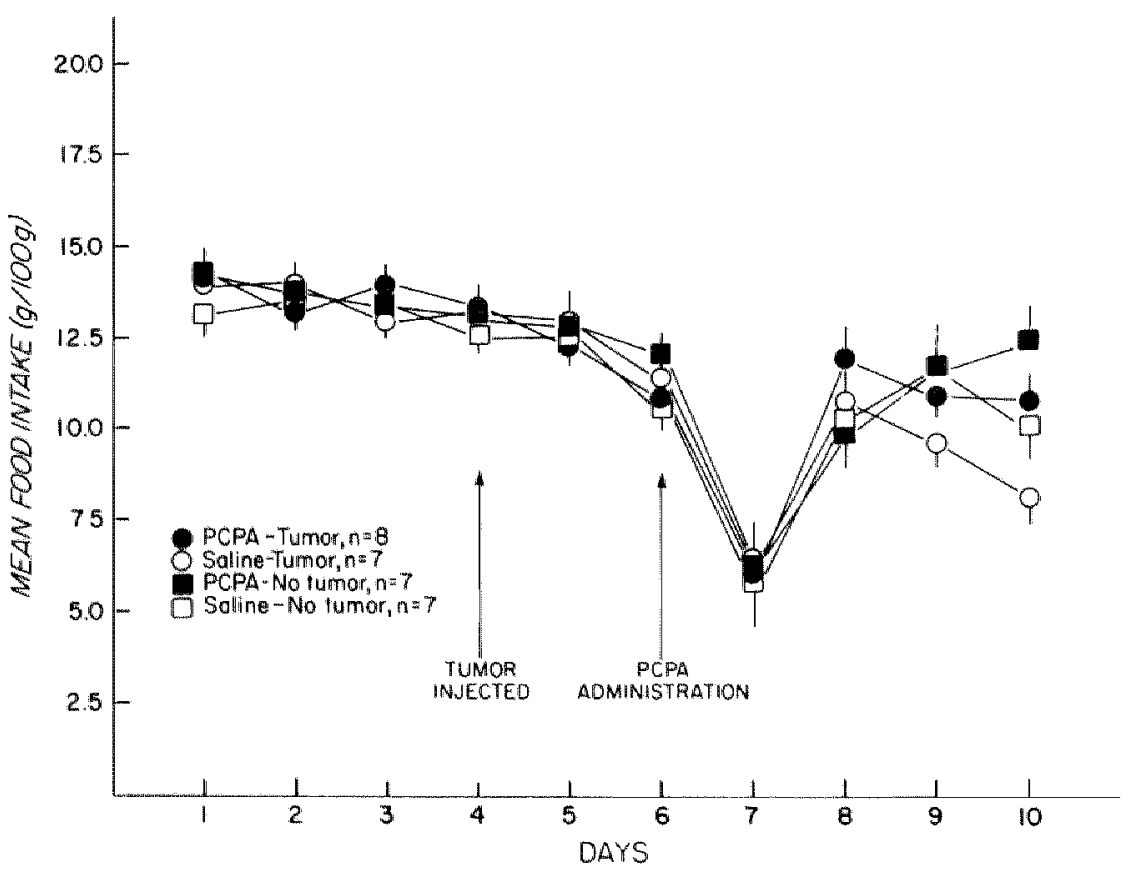

Fig. 9.3.4 Mean (- SEM) daily ad libitum food consumption of tumor bearing (circles) and non tumor bearing control (squares) rats following the bilateral intraventricular administration of DL-p-CPA methylester (2 $\mathrm{mg}$ in $8 \mu \mathrm{l}$, clased symbols) or normal saline $(8 \mu \mathrm{l}$, open symboils) in Exp. 2 . All rats were sacrificed 6 days after im. inoculation of $5 \times 10^{4} W_{256}$ tumor cells or normal saline. 
Table 9.3.-1 Mean ( \pm SEM) plasma tree and total TRP levels and brain tryptophan (TRP). 5-HT and 5-HIAA levels, of tumor bearing and control rats 7 days after the ivt. injection of p-CPA or saline. (Experiment 1 ).

\begin{tabular}{|c|c|c|c|c|c|}
\hline & $\begin{array}{l}\text { Plasma } \\
\text { Free TRP } \\
\text { (nmol/ml) }\end{array}$ & $\begin{array}{l}\text { Total TAP } \\
\text { (nmol/ml) }\end{array}$ & $\begin{array}{l}\text { Brain } \\
\text { TRP } \\
(\mu g / g)\end{array}$ & $\begin{array}{l}5 \cdot H \pi \\
(n g / g)\end{array}$ & $\begin{array}{l}5-H 1 A A \\
(m g / g)\end{array}$ \\
\hline $\begin{array}{l}T B-p-C P A \\
(n=7)\end{array}$ & $4.6 \pm 0.4^{a}$ & $8.9 \pm 1.3^{a}$ & $3.0 \pm 0.3$ & $655 \pm 19$ & $51.4 \pm 25^{\circ}$ \\
\hline $\begin{array}{l}\text { TB-Sal } \\
(n=7)\end{array}$ & $4.6 \pm 0.2^{a}$ & $9.7 \pm 1.0^{a}$ & $3.3 \pm 0.1^{a}$ & $700 \pm 14$ & $617+18^{a}$ \\
\hline $\begin{array}{l}F F-p-C P A \\
(n=7)\end{array}$ & $2.9 \pm 0.2$ & $13.1 \pm 1.6$ & $2.7 \pm 0.1$ & $629 \pm 27^{b}$ & $424 \pm 36^{b}$ \\
\hline $\begin{array}{l}\text { FF-Sal } \\
(n=8)\end{array}$ & $3.1 \pm 0.3$ & $15.6 \pm 1.0$ & $2.6 \pm 0.1$ & $721 \pm 20$ & $521 \pm 19$ \\
\hline
\end{tabular}

Post hoc t-tests indicated decreased 5-HT and 5-HIAA in both FF-P-CPA $(p<0.01)$ and TB-p-CPA $(p<0.05)$ groups. Although the tumor treatment did not elevate brain $5-H T$, levels of 5 -HIAA were significantly increased in tumor bearing groups $(F(1,25)=6.89, p<0.05)$.

Analysis of brain catecholamine data (Table 9.3.-2) indicated that the p-CPA treatment significantly reduced $N E(F(1,25)=6.85 ; p<0.05)$ while not affecting $D A$. The tumor treatment had no significant effect on brain catecholamines.

Table 9.3-2 Mean ( \pm SEM) brain 5-HT, 5-HIAA, NE and DA levels of tumor-bearing and contral rats 4 days after the ivt. injection of $p$-CPA or saline. (Experiment 2).

\begin{tabular}{|c|c|c|c|c|}
\hline & $\begin{array}{l}5-H T \\
(n g / g)\end{array}$ & $\begin{array}{l}\text { 5-HIAA } \\
(\mathrm{ng} / \mathrm{g})\end{array}$ & $\begin{array}{l}\text { NE } \\
(n g / g)\end{array}$ & $\begin{array}{l}D A \\
(n g / g)\end{array}$ \\
\hline $\begin{array}{l}\text { TB-p-CPA } \\
(n=8)\end{array}$ & $597 \pm 25^{a b}$ & $498 \pm 39^{b}$ & $485 \pm 11^{c}$ & $1,014+34$ \\
\hline $\begin{array}{l}\text { TB-Sal } \\
(n=7)\end{array}$ & $699 \pm 35$ & $597 \pm 24$ & $510 \pm 7$ & $992 \pm 29$ \\
\hline $\begin{array}{l}F F-p-C P A \\
(n=7)\end{array}$ & $568 \pm 47^{a}$ & $387+42^{a}$ & $490 \pm 11^{\circ}$ & $987 \pm 24$ \\
\hline $\begin{array}{l}\text { FF-Sal } \\
(n=7)\end{array}$ & $718 \pm 21$ & $535 \pm 29$ & $519 \pm 12$ & $1,000 \pm 23$ \\
\hline
\end{tabular}




\subsection{Discussion}

The data from these experiments support a role of brain 5-HT in the mediation of cancer anorexia. Thus, in agreement with results from previous experiments, brain hevels of TRP. and 5-HIAA, were significantly elevated in anorectic TB rats. The changes in peripheral circulating levels of total and free TAP also rellect previous results.

The theory of involvement of 5-HT systems in cancer anorexia is supported by the effectiveness of $\mathrm{p}$-CPA treatment in delaying the onset of anorexia for 2 days. Although p-CPA hyperphagia (Breisch, 1976: Hoebel, 1978) has recently been demonstrated to be non specific (Coscina, 1978; Mackenzie, 1979), the absence of hyperphagia in p-CPA treated FF rats emphasizes the differential response of the TB group. Furthermore, the bilochemical changes following p-CPA treatment were not as severe as previously reported (Breisch, 1976). Thus the p-CPA injections appeared to normalize 5-HIAA levels in TB rats. Therefore, this treatment may have corrected a biochemical abnormality that resulted from the presence of a growing tumor.

Although the magnitude of the effect was quite small, p-CPA treatment did significantly reduce CNS-NNE levels. Since NE has been reported to stimulate eating (Grossman. 1962; Leibowitz, 1978'), a decrease by p-CPA would counteract a possible facilitory effect on food intake of this drug through inhibition of the serotonin system (Saller. 1976). This observation may explain the moderate effect of the drug on food intake in the condition here under investigation, as compared to the reports by Breisch (1976). Other reports however have NE implicated in the mediation of satiely (Margules. 1972) therefore the reduction of NE concentrations by the administration of p-CPA could also be the mechanism for the delay in the onset of anorexia in TB-p-CPA animals.

Additional mechanisms causing cancer anorexia must thus be considered, also because TB-p-CPA rats did eventually become anorectic, in the presence of decreased 5-HAAA concentrations.

\subsection{Conclusions}

1. Inhibition of TRP-hydroxylase by the ivt. administration of P-CPA in TB and FF animals results in significant deptetion of brain $5-\mathrm{HT}$, illustrated by significant lower brain $5-\mathrm{HT}$ and 5-HIAA levels.

2. The depletion of central $5-H T$ is in TB animals associated with a significant delay in the onset of cancer induced anorexia by two days, while no hyperphagia is observed in FF-p-CPA animals compared to FF-Sal animals.

3. A significant decrease of bran NE levels 4 days after the ivt. administration of p-CPA may be the reason for only a moderate behavioral response to this drug treatment

4. If NE were to mediate satiety the observed reduction of NE in TB-p-CPA animals could account for the delay in the onset of anorexia.

5. The presence of significant anorexia in TB-p-CPA animals in combination with still significantly decreased 5-HIAA levels in these animals suggests additional mechanisms being active in the mediation of cancer anorexia. 


\section{Chapter 10}

\section{The effect of methysergide on food intake in anorectic cancer bearing rats}

\subsection{Introduction}

To more fully investigate a role for 5-HT in the mediation of cancer anorexia, we administered the putative 5mHT receptor blocker methysergide (M) (Jespersen, 1970; Panksepp, 1979) intraventricularly across two experiments, employing MCA tumor bearing Fisher 344 rats implanted with ivt. positioned cannulae. The ip. or sc. administration of this drug, in doses varying from 1.25 to $5.0 \mathrm{mg} / \mathrm{Kg} \mathrm{BW}$, has been reported (Blundell, 1973; Clineschmidt, 1974; Barrett, 1975) to inhibit the appetite suppressant activity of fenfluramine, which presumably acts through a serotonergic mechanism (Funderburk, 1971; Blundell, 1973; Jespersen, 1973; Clineschmidt, 1974).

\subsection{Materials and methods}

\section{Design}

Sixtyone adult male Fisher 344 rats were used across two experiments. MCA tumors were induced in 31 of these animals after they had recovered from the cannulae implantation procedure as indicated by the restoration of normal food intake. The other 30 rats served as saline-injected controls. In experiment 1 , where animals were divided into the following groups: TB-M $(n=8)$, TB-Sal $(n=7), F F-M(n=8)$ and FF-Sal $(n=7)$, reduced food intake in TB was first observed on day 25 . Therefore, methysergide or saline was injected ivt. on days 29 . 31 and 33 .

In experiment 2, again employing four groups: TB-M $(n=8)$, TB-Sal $(n=8)$. FF-M $(n=8)$, FF-Sal $(n=7)$, reduced food intake in TB rats was well established on day 28 . After a sham injection artificial CSF (Yaksh, 1976) on day 31, the test drug or artificial CSF was infused on days 33 and 35.

Rats in experiment 1 were sacrificed on day 40 , while im experiment 2 all rats were sacrificed on day 37 post tumor implantation. One TB-Sal animal in experiment 1 died just prior to sacrifice and biochemical analyses were consequently performed on 6 animals in this group.

\section{Intraventricular injection of methysergide}

Prior to tumor induction $24 \mathrm{ga}$ cannulae were stereotaxically implanted unilaterally at the following coordinates (Pellegrino and Cushman Atlas, 1967) relative to bregma: $A=-0.2$. $L \pm 1.5$ and $V=-4.5 \mathrm{~mm}$, to allow repeated ivt. injections.

In experiment one $10 \mu$ of methysergide in incrementing doses of 25,50 and $100 \mu \mathrm{g}$ was injected in 8 of the 15 rats in each group on days 29,31 and 33 , while the other 7 rats in each group received an equal volume normal saline on these days. On day 27 all rats received $10 \mu \|$ saline to adapt them to the ivt. injections. In experiment 2 all rats in each group were infused with $10 \mu$ artificial CSF on day 31 in order to acclimate them to the ivt. injections. On days 33 and $35,100 \mu \mathrm{g}$ methysergide or artificial CSF (10 $\mu 1)$ was administered to half of each group. Methysergide was obtained from Sandoz (East Hanover. N.J.). Each injection was delivered through a 31 ga needle using a $50 \mu l$ syringe (Hamilton 


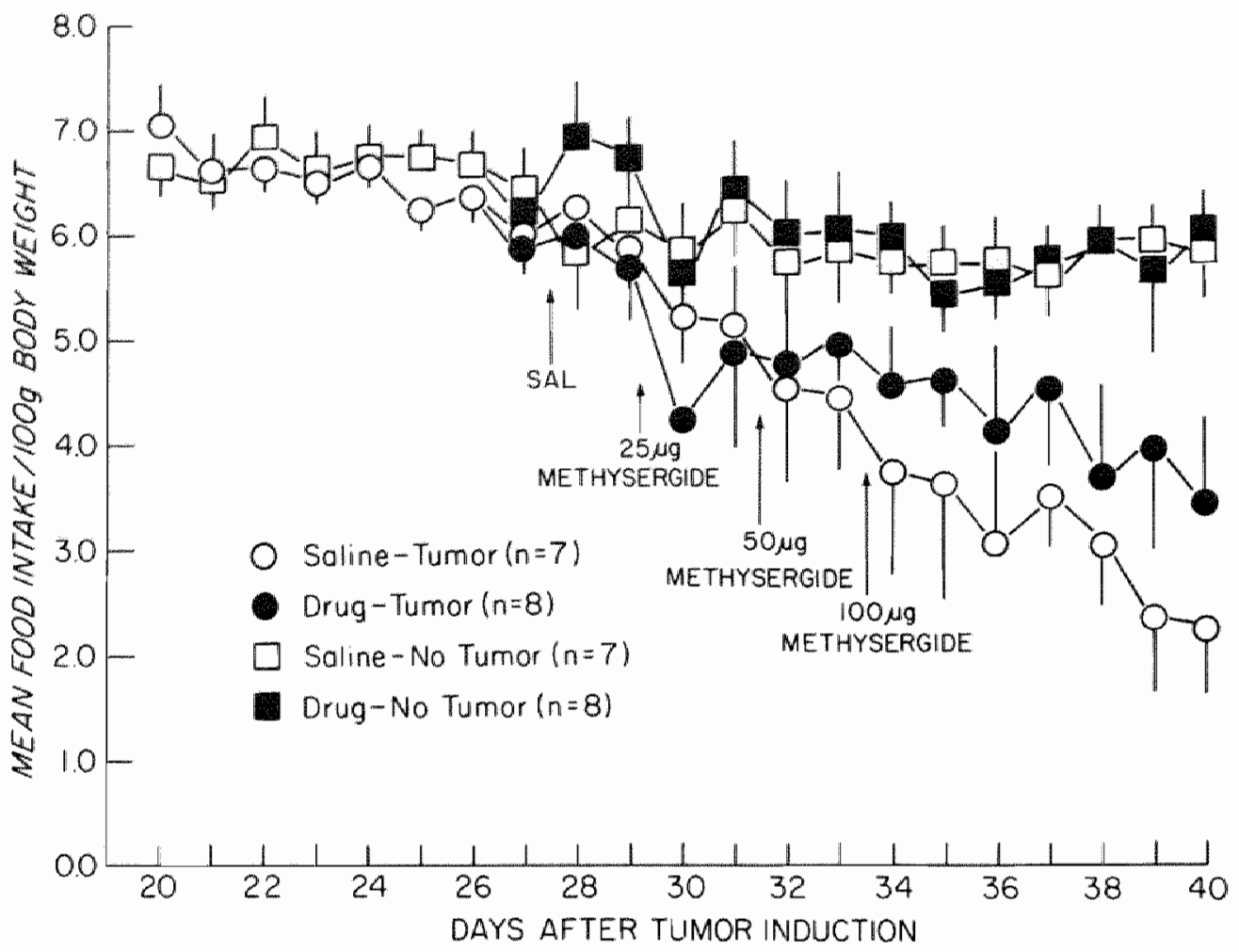

Fig. 10.3.1 Mean (I SEM) daily food intake by adult male MCA tumor bearing (circles) and control (squares) rats following the unilateral intraventricular (ivt.) injection of saline (open symbols) or various doses of the putative serotonin receptor blocker. methysergide (closed symbols). On day 27 all rats were injected (ivt.) with normal saline $(10 \mu l)$, while the drug treatments were initiated on day 29 .

Table 10.3.-1 Mean ( \pm SEM) whole brain levels of TRP, 5-HT, 5-HIAA, NE and DA in MCA sarcoma bearing (TB) and control (FF) rats following ivt administration of increasing doses of methysergide or control injections of normal saline. At the bottom $p$ values of 2-way ANOVA tests.

\begin{tabular}{|c|c|c|c|c|c|}
\hline Group & $\begin{array}{l}\text { TRP } \\
(\mu g / g)\end{array}$ & $\begin{array}{l}5 m \mathrm{HT} \\
(\mathrm{ng} / \mathrm{g})\end{array}$ & $\begin{array}{l}5-H I A A \\
(n g / g)\end{array}$ & $\begin{array}{l}\mathbb{N E} \\
(n g / g)\end{array}$ & $\begin{array}{l}D A \\
(n g / g)\end{array}$ \\
\hline $\begin{array}{l}\text { TB-Methysergide } \\
(\mathrm{n}=8)\end{array}$ & $3.49+0.4$ & $902 \pm 43$ & $541 \pm 32$ & $613 \pm 23$ & $1,217 \pm 36$ \\
\hline $\begin{array}{l}\text { TQ-Saline } \\
(n=6)\end{array}$ & $4.14 \pm 0.6$ & $970 \pm 57$ & $571 \pm 38$ & $580+17$ & $1,209 \pm 37$ \\
\hline $\begin{array}{l}\text { FF-Methysergide } \\
(n=8)\end{array}$ & $2.99 \pm 0.1^{b}$ & $859 \pm 20$ & $402 \pm 9^{a}$ & $550 \pm 15^{b}$ & $1,164 \pm 29$ \\
\hline $\begin{array}{l}\text { FF-Saline } \\
(n=7)\end{array}$ & $3.09 \pm 0.1^{b}$ & $894 \pm 26$ & $417 \pm 14^{a}$ & $553 \pm 14^{\circ}$ & $1,217 \pm 49$ \\
\hline
\end{tabular}


Co., Reno, Nev.) connected to a repeating depenser (Hamilton Co. Reno, Nev.) at a rate of 4 fil/min.

\section{Dissection and biochemical analysis}

Following sacrifice by decapitation, brains were rapidly removed, hemisectioned and frozen in liquid nitrogen in the first experiment. Brain TRP, 5-HT, 5-HIAA. NE and DA levels were determined according to techniques described in chapter 2

\subsection{Results}

Mean food intake in the first, dose response, experiment is shown in Fig. 10.3-1. As may be seen significant anorexia in TB rats as compared to NTB rats $(p<0.05)$ was first observed on day 25. Although the $100 \mu \mathrm{g}$ dose appeared to have a positive effect, in that it maintained eating in TB animals, variability in the obtained food intake data precluded achievement of statistical significance of this effect.

Analysis of biochemical assay results by means of a 2 -way ANOVA revealed significant

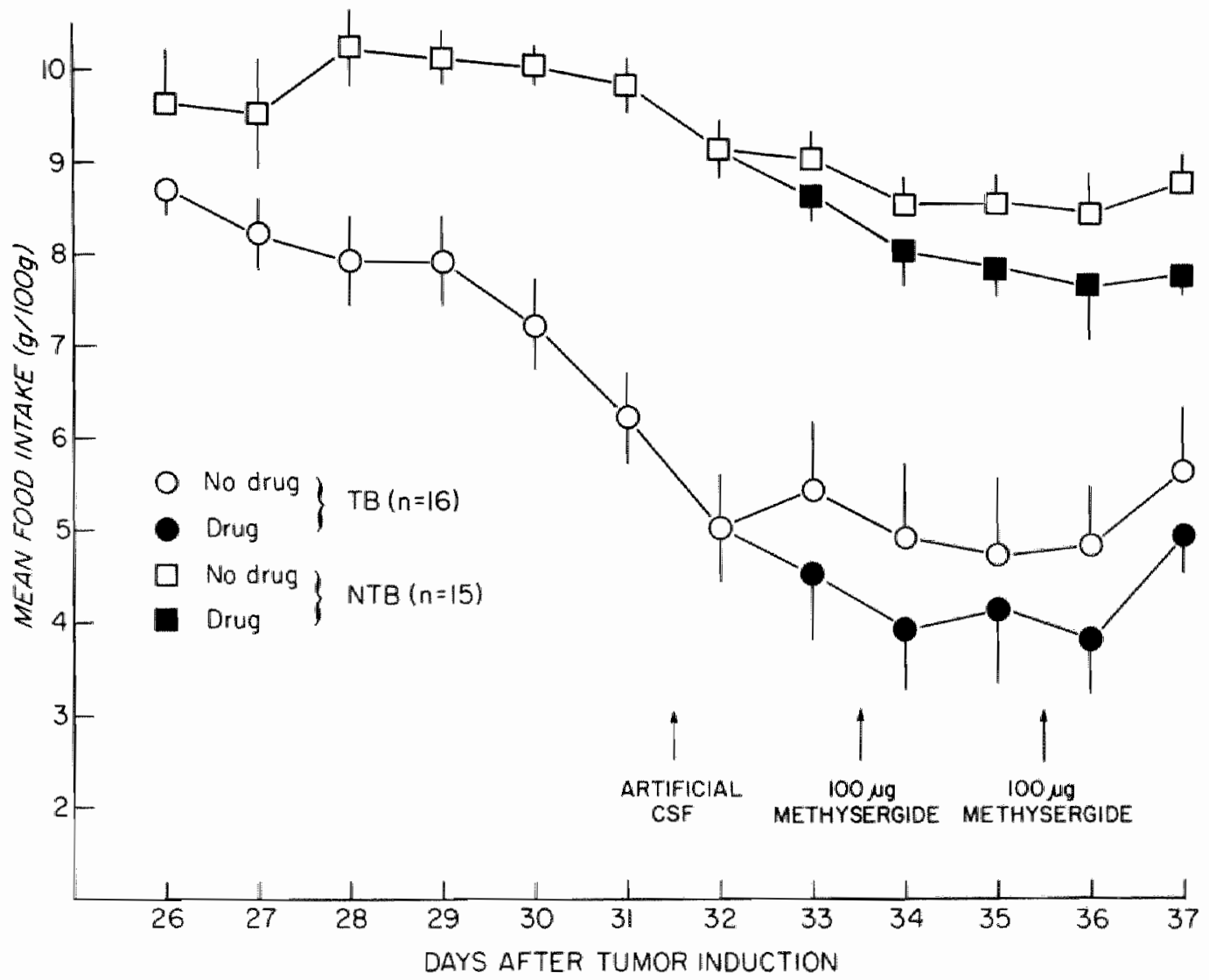

Fig. 10.3.-2 Mearr (i. SEM) daily tood intake by adult male MCA fumor bearing (circles) and control (squares) rats lollowing the unilaterall intraventricular (ivt.) injection $(10 \mu$ ) of artifheial cerebrospinal fluld (CSF) (open symbols) or $100 \mu \mathrm{g}$ of methysergide (closed symbols). Control rats from both groups continued to be injected (ivt.) with CSF on days 33 and 35. 
changes across groups for TRP $(F(1,27)=5.22 ; p<0.05), 5-H A A,(F(1,27)=34.49 ; 0<0001)$ and $N E(F(1,27) \cdots 4.81, p<0.05)$. As was to be expected with the use of this drug no changes were observed comparing data across drug treatments (Table 10.3.-1.)

In an effort to achieve statistical significance ot the trend towards maintained eating in $T B$ animals by methysergide treatment, $100 \mu \mathrm{g}$ methysergide was infused ivt. on 2 days in a second experiment of identical design. When infusions started on day 31 post tumor implantation anorexia was well developed. As may be observed from Fig. 10.3.-2 methysergide treatment failed to increase eating in TB animals to a significant degree.

\subsection{Discussion}

Methysergide has been reported to antagonize 5-HT induced behavior (Corne, 1967; Mawson, 1970; Barrett, 1975), as well as fenfluramine induced anorexia (Jespersen, 1970; Blundell, 1973; Clineschmidt, 1974). In these experiments, employing the ivt. administration of this 5-HT receptor blocker, no effect on anorexia in TB animals could be recorded, although analysis of biochemical data in the first experiment indicated increased levels of brain TRP and 5-HIAA in these animals.

There could be several reasons for the discrepancy with the previous suggestions of serotonin inwolvement in the mediation of cancer anorexia, one being that our assumption to this effect was wrong, and the changes observed a concomitant effect of diminished feeding (Curzon, 1972; Perez-Cruet, 1972).

Secondly, no experience with the ivt. administration of this drug in relation to food intake is available, and therefore it may be argued that a suboptimal dosage was administered. Thirdly, a biphasic action of methysergide on fenfluramine induced anorexia has been described, first antagonizing fenfluramine induced anorexia, and after approximately $4 \mathrm{hr}$ starting to potentiate the drug induced anorexia. The above observation may be of importance reviewing the results of this experiment, in which $24 \mathrm{hr}$ food intake was. evaluated.

Finally, recent reports describe 2 distinct serotonin receptors (Haigler, 1977; Bennett, 1978 Peroutka, 1979, 1981), designated serotonin 1 and serotonin 2 , that mediate inhibition and excitation respectively. Since the serotonin behavioral syndrome, that includes resting tremor, hindlimb abduction, snake tail side-to-side head weaving and head twitching. appears to involve serotonin 2 receptors, it may reflect excitatory synaptic actions of central serotonin (Peroutka, 1981).

Synaptic antagonistic effects of methysergide are more potent at serotonin 2 receptors. while methergoline, which blocks both inhibition and excitation of serotonin, displays high affinity for both receptors (Peroutka, 1981). This drug has been associated with 10-30 times more antagonistic activity on fenfluramine induced anorexia (Mawson, 1970; Clineschmidt, 1974). Thus, in accordance with the observed lack of effect (Jespersen, 1973), moderate (Clineschmidt, 1974) or dualistic blocking effect (Blundell, 1973) of methysergide on fenfluramine induced anorexia, it could be hypothesized that fenfluramine induced inhibition of food intake behavior is transmitted via the inhibitory, serotonin 1 receptor. This interpretation would explain the lack of, or minimal response to methysergide blockade, and the better response to methergoline blockade, as reported in literature (Mawson, 1970; Clineschmidt, 1974).

In conjunction with the above, it could further be hypothesized that cancer anorexia is also transmitted wia the inhibitory serotonin 1 receptor. If so, the lack of effect on food intake by methysergide blockade, as demonstrated in these experiments, could be explained by the low affinity of methysergide for serotonin 1 receptors. 


\subsection{Conclusions}

1. Across two experiments the ivt administration of the putatiwe serotonin blocker methysergide up to a dose of $100 \mu \mathrm{g}$, failed to increase food intake in TB animals in the presence of increased central serotonin activity in these animals.

2. Elewated brain NE levels in TB animals may inadequately signal satiely. 


\section{The effect of 5,7-dihydroxytryptamine on serotonin metabolism and food intake in cancer bearing rats}

\subsection{Introduction}

The drug 5,7-dihydroxytryptamine (5,7-DHT) has been reported to inhibit TRP-hydroxylase activity (Baumgarten, 1973 ${ }^{\text {a }}$, and to cause a degeneration of serotonergic neurons and "to a lesser extent of noradrenergic neurons (Baumgarten, 1973") in the CNS. The depletion of NE is selectively antagonized by agents that inhibit uptake of 5,7-DHT into NE neurons such as desmethylimipramine (DMU) (Breese, 1975). Thus, pretreating experimental animals with DMI would limit lesions induced by the administration of 5,7-DHT to serotonergic neurons. Saller and Stricker (1976) reported hyperphagia and body weight gain in juvenile male rats. after administration of $200 \mu 95,7-\mathrm{DHT}$ intraventricularly following pretreatment with $25 \mathrm{mg} / \mathrm{kg}$ DMI. If this treatment resulted in 60-80\% depletion of forebrain serotonin, without affecting NE levels, hyperphagia was obsarved. To accomplish serotonin depletion in cancer bearing and control rats, and thus further investigate a possible role of this neurotransmitter in the mediation of cancer anorexia, we used 5.7-DHT in immature female SD rats, that were to be inocullated with $W_{256}$ tumors.

\subsection{Materials and methods}

\section{Design}

Twenty eight 40-60 g female SD rats were, after pretreatment with DM1, divided into two groups: 16 rats received 5,7-DHT intracisternally (ict.), while the remaining rats received an equal volume of normal saline in an identical manner. Two weeks later $W_{256}$ tumors were induced in 7 rats of the 5,7-DHT treated group, and 5 animals of the Sal injected group. Five of the TB animals died prior to sacrifice on day 9 post tumor implantation "the day the experiment was terminated.

\section{Tumor induction}

Because the animals had become slightly larger than usual at the time tumors were to be induced, it was decided to increase the number of tumor cells to be injected, to $1 \times 10^{\text {sin }}$ $0.1 \mathrm{cc}$.

\section{Drug treatment}

All rats received $30 \mathrm{mg} / \mathrm{kg}$ desmethylimipramine ip. (Merrell $\mathrm{Co}$. Cincinnat, $\mathrm{OH}$ ) followed in half an hour by ict. injection of $100 \mu \mathrm{g}$ 5.7-DHT (Sigma Chem. Co., St. Louis, MO) contained in $20 \mu$ of normal saline plus $0.5 \%$ ascorbic acid in the test drug treated group, or an equal wolume $(20 \mu 1)$ normal saline plus ascorbic acid in controls.

Ascorbic acid was added to the medium to preserve the drugs efficacy by delaying its oxidation (Baumgarten, 1976). 
adminigtrationj, brains were rapidly removed, hemisectioned and frozen in liquid nitrogen for deterimination of TRP, 5山HT, 5-HIAA, NE and DA, using techniques described in chapter 2.

\subsection{Results}

As may be observed from fig. 11,3-1, the ict. injection of $5,7-\mathrm{DHT}$ did not affect the anorectic response of TB rats, nor did it lead to hyperphagia in the drug treated FF animals Since 5 of the TB animals died prior to sacrifice on day 9 , the statistical evaluation of food intake data was conducted over days 4 through 8 . A repeated measures ANOVA confirmed the absence of a drug effect, while indicating significant tumor $(F(1,29)-32.2 p<0.01)$, days $(F(4,116)=23,8 ; p<0.01)$ and tumor $x$ days $(F(4,116)=4.14, p<0.01)$ effects. Thus, food intake by both TB groups was progressively decreased as the tumors grew.

Stetistical analysis of biochemical data, employing a 2 -way analysis of variance, indicated changes in TRP $(F(1,24)=7.30 ; p<0.05), 5-H I A A(F(1,24)=18.37 ; p<0.001)$ and $N E(F(1,24)=8.72$; $p<0.01)$ across tumor treatment. Comparisons across drug treatment revealed significant changes of 5-HT $(F(1,24)=82.21 ; p<0.001)$ and 5-HIAA $(F(1.24)=116.15 ; p<0.001)$, with NE levels remaining unaffected. A significant tumor $x$ drug interaction was observed only within $5-H I A A$ results, $(F(1,24)=8.92 ; p<0.01)$. Although tumor treatment did not alter brain $5-H T$, post hoc $t$-tests indicated that levels of 5 -HIAA were significantly $(p<0.001)$ elevated in untreated TB animals. Drug treatment was associated with significantly $(p<0.001)$ reduced levels of

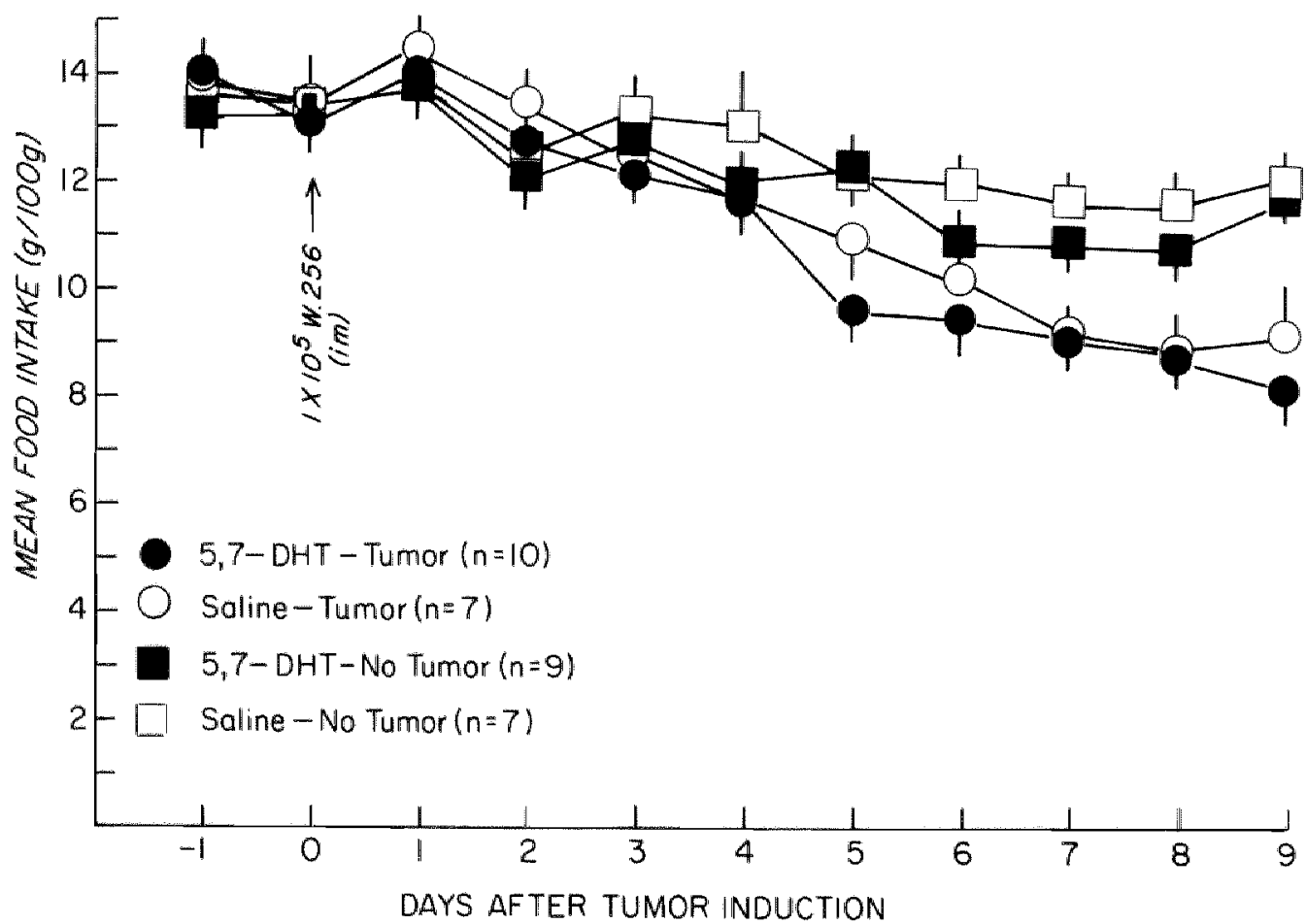

Fig. 11.3-1 Mean ( 1 SEM) daily food intake by immature female rats bearing Walker 256 tumors (circles) and corresponding saline-injected control (squares) rats. Two weeks prior to the induction of tumors, rats from each group received intracisternal (ict.) injections of the serotonergic neurotoxic drug. 5,7-dihydroxytryptamine $(100 \mu \mathrm{g})$ (closed symbols) or contral injections $(20 \mu l)$ of normall saline (open symbols). 
Table 11.3.-1 Mean (1 SEM) whole brain levels of TRP, 5-HT, 5-HIAA, NE and DA in W.5. tumor bearing (TB) and control (FF) rats following depletion of indoles by ict administration of 5,7-dihydroxytryptamine (5,7-DHT) or control injections of normal saline (Sal). At the bottom $p$ values of post hoc t-tests.

\begin{tabular}{llllll}
\hline & $\begin{array}{l}\text { TRP } \\
(\mu \mathrm{g} / \mathrm{g})\end{array}$ & $\begin{array}{l}5-\mathrm{HT} \\
(\mathrm{ng} / \mathrm{g})\end{array}$ & $\begin{array}{l}5-\mathrm{HIAA} \\
(\mathrm{ng} / \mathrm{g})\end{array}$ & $\begin{array}{l}\mathrm{NE} \\
(\mathrm{ng} / \mathrm{g})\end{array}$ & $\begin{array}{l}\mathrm{DA} \\
(\mathrm{ng} / \mathrm{g})\end{array}$ \\
\hline $\begin{array}{l}\text { TB-5,7-DHT } \\
(\mathrm{n}=7)\end{array}$ & $3.24 \pm 0.50$ & $356 \pm 4^{\mathrm{c}}$ & $315 \pm 34^{\mathrm{cd}}$ & $416 \pm 34^{\mathrm{a}}$ & $867 \pm 48$ \\
$\begin{array}{l}\text { TB-Sal } \\
(\mathrm{n}=5)\end{array}$ & $3.35 \pm 0.43$ & $712 \pm 33$ & $676 \pm 22^{\circ}$ & $424 \pm 38$ & $838 \pm 67$ \\
$\begin{array}{l}\text { FF-5,7-DHT } \\
(\mathrm{n}=9)\end{array}$ & $2.13 \pm 0.02$ & $405 \pm 31^{\mathrm{c}}$ & $266 \pm 21^{\mathrm{C}}$ & $503 \pm 19$ & $911 \pm 24$ \\
$\begin{array}{l}\text { FF-Sal } \\
(n=7)\end{array}$ & $2.61 \pm 0.28$ & $711 \pm 36$ & $474 \pm 21$ & $484 \pm 16$ & $841 \pm 35$ \\
\hline
\end{tabular}

$a=p<0.05$ vs. FF groups; $b=p<0.001$ vs FF groups; $c=p<0.001$ vs non depleted controls; $d=p<0.001$ vs drug + condition controls

5-HT and 5-HIAA in both TB and NTB groups, as compared to non depleted controls. Strikingly, 5,7-DHT treated TB animals were anorectic, although 5-HIAA levels were significantly $(p<0.001)$ reduced compared to non drug treated FF animals. Furthermore, post hoc t-tests indicated that within the TB group animals displayed significantly $(p<0.05)$ decreased NE levels.

\subsection{Discussion}

The data from this experiment are in agreement with results from previous experiments in so far, that brain levels of TRP and 5-HIAA were significantly elevated in anorectic TB-rats. In contrast to findings reported by Saller and Stricker (1976), we did not observe hyperphagia or body weight gain in drug treated NTB animals. However, several other investigators have not been able to replicate Saller and Stricker's lindings; thus Coscina (1978 ${ }^{\text {b }}$. Hoebel (1978) and Meyers (1978) did not observe hyperphagia or body weight glain, while Breese (1975) observed reduced body weights in 5,7-DHT treated rats, apparently because of 'a reduction in suckling by the rat pups'. Coscina $\left(1978^{\circ}\right)$ extensively reviewed possible reasons for the discrepancy between his and Saller's observations but found none valid. In our experiments the same route of drugadministration was used as Coscina's, and similar 5-HT depletions (43-50\%) and even greater 5-HIAA decrements (44-53\%) were obtained. The above observed depletions however did still not reach the 60-70\%, or even $80 \%$, thought by Saller and Stricker (1976) to be essential to cause hyperphagia. In contrast to this opinion. Hoebel (1978) could not replicate hyperphagia, even in the presence of 5.HT depletions greater than $80 \%$, and normal NE levels.

The above evidence, that serotonin depletion does not necessarily disinhibit feeding (Hoebel, 1978; Samanin, 1980 ${ }^{\mathrm{b}}$ ), makes interpretation of our observations in tumor bearing animals more difficult. Lack of effect on food intake in tumor bearing animals, in the presence of significant 5-HT and 5-HIAA depletions, may suggest, that serotonin is not 
inwolved in the mediation of cancer anorexia, but may also reflect the ineffectiveness of this drug in intervening in any behavior, traditionally thought to be influenced by serotonin (Lorens, 1978). This ineffectiveness is not well understood (Coscina, 1978) and may result from denervation supersensitiwity within 5-HT systems. Another explanation may lie in that this drug does not deplete functionally active $5 . H T$.

The rolle of NE in the observed behavioral results in this experiment is unclear: the $\pm 15 \%$ decreased levels of NE in TB animals, unaffected by 5,7-DHT treatment, are probably unimportant, since they do not amount to the $30 \%$ depletions, implicated by Saller (1976) in the lack of thyperphagic response to 5,7-DHT treatment. Obviously more investigations to clarify the roles of both 5,7-DHT and NE in the development of cancer anorexia are indicated.

\subsection{Conclusions}

1. The observed changes in TRP and 5-HT indoles, and food intake behavior in TB animals replicate our previous results.

2. Serotonin depletion by administration of $5,7-D H T$ does not disinhibit feeding in either control or TB animals, thus making a rolle for 5-HT in the mediation of cancer anorexia uncertain.

3. NE reduction in TB animals requires additional investigation. 


\section{The effect of intrahypothalamic administration of norepinephrine with and without manipulation of the serotonin system on food intake in tumor bearing animals}

\subsection{Introduction}

In 1962 Grossmann first demonstrated, that norepinephrine (NE), when injected through chronic cannulae directly into the perifornical hypothalamus of satiated rats, could ellicit a vigorous feeding response. His results were confirmed by Leibowitz $\left(1976,1978^{b}\right)$, who established a dose response curve for NE induced eating, focusing stimulatory effects on the paraventricular hypothalamus ( $\mathrm{PVH}$ ).

Although previous research has suggested that declined food intake in cancer disease occurs in the presence of normal hypothalamic mechanisms, this research was based on gross lesions of large areas of the ventromedial hypothalamus (VMH) or lateral hypothalamus. Thus, Baillie (1965) described the onset and quality of tumor induced anorexia in SD rats being unaltered in VMH lesioned, hyperphagic rats implanted with the Walker 256 tumor. In addition, lesions to the lateral hypothalamus produced true aphagia and adipsia, suggesting normal control mechanisms being functional in this area. Also, the feeding response to exogenous insulin, which is thought to be mediated through the hypothalamus, is not impaired in the presence of $W_{256}$ tumors (Morrison, 1975).

No research of chemical stimulation of the PVH in TB animals has been attempted however. We therefore investigated eating elicited by the injection of NE in the PVH in MCA TB and FF animals. In addition we investigated the role of $5-\mathrm{HT}$ as inhibitory factor (Leibowitz, 19789 by examining the effects of intrahypothalamic pretreatment with methysergide, and of 5-HT depletion by parachloroamphetamine (PCA) on eating, elicited by NE stimulation of the PVH. PCA apparently achieves depletion of brain 5-HT by irreversibly inhibiting TRP-hydroxylase, by inhibiting high affinity 5-HT uptake and by its selective neurotoxicity (Sanders-Bush, 1977).

\subsection{Materials and methods}

\section{Design}

Forty nine male Fisher $344( \pm 250 \mathrm{~g})$ rats were used across two experiments. In the first experiment MCA tumors were induced in 12 animals, while an equal number served as sham injected controls. Cannulae were stereotaxically implanted into the PVH, and on day 33 , when anorexia was well developed, biochemical manipulations started. Injections in the hypothalamus were repeated on days $35,37,39$ and 41 , when all rats were sacrificed. In the second experiment MCA tumors were induced in 17 rats, with 8 rats serving as controls. One week after tumor induction $B$ TB rats were injected with PCA $(10 \mathrm{mg} / \mathrm{kg}) \mathrm{ip}$. Biochemical manipulations took place on days $18,20,25,27$ and 29 , followed by sacrifice of all rats on day 32 .

In these experiments eating was assessed for one hour following the control intrahypothalamic (int.) injection of $1 \mu$ l of normal saline (NS). Immediately thereafter NE (Sigma Chemical Co. St. Louis, MO), dissolved in $1 \mu$ INS, was injected and eating was again assessed for one hour. Presented values represent food intake in the drug period 
minus lood intake in the $\mathrm{NS}$ period. Twenty four hr food intake was also recorded and is presented as $\mathrm{g} / 100 \mathrm{~g} \mathrm{BW}$.

\section{Intrahypothalamic injections of chemicals}

Cannulae (24 ga) were stereotaxically implanted into the PVH unilaterally, at the following coordinates relative to bregma: $A=-0.5 \mathrm{~mm}, L= \pm 0.5 \mathrm{~mm}$ and $V=-7.2 \mathrm{~mm}$ (Pellegrino and Cushman Atlas).

In the first experiment. NE $(24$ nmol, $1 \mu \|)$ was first infused on day 33 , when TB rats displayed significant anorexia, and repeated on days 35 and 37 . NE infusion on days 39 and 41 was preceded $(5 \mathrm{~min})$ by iht. injection of either methysergide $(5 \mu \mathrm{g}, 1 \mu \mathrm{ll}$ ) or normal saline $(1 \mu l)$ in both groups in a counterbalanced order.

Because in the previous experiment NE induced eating appeared to be consistently although slightly reduced in TB animals, the second experiment investigated a doseresponse relationship of $\mathbb{N E}$ to eating in TB animals. To allow further investigation of a serotonin involvement, in this experiment 8 TB rats were injected ip. with $P C A(10 \mathrm{mg} / \mathrm{kg})$

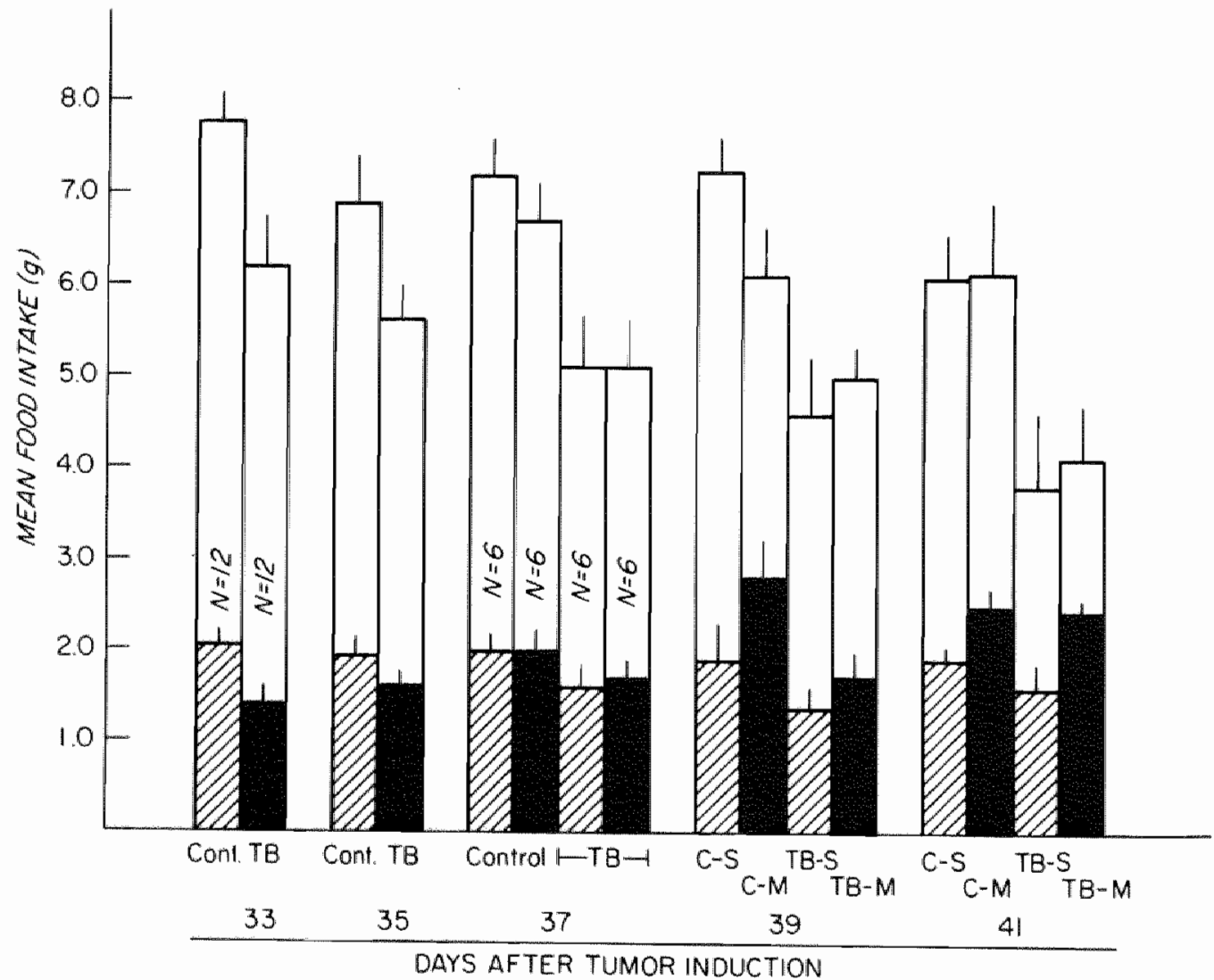

Figure 12.3.-1 Mean (t. SEM) dally food intake ( $9 / 100 \mathrm{~g}$ body weight, open bars) and norepinephrine (NE)-induced eating (total g. shaded bars) of adult male MCA sarcoma bearing (TB) and control (Cont. or C.) rats. Cannulae were implanted into the periventicular area of the hypothalamus of all rats. Shaded bars represent eating during the hour following intrahypothalamic (iht.) injection of 24 nmol of NE minus eating during the previous hour following the control injection ( 1 ul) of normal saline. On days 39 and 41 each group was halved lor pretreatment (5 min) with iht. methysergide $(M, 5 \mu g / \mu 1)$ or inormal satine (1 $\mu$ l). 
(Sigma Chemical Co. St. Louis, MO) one week after the induction of MCA tumors. On days $18,20,25,27$ and 29 NE was infused into the PVH, and eating response of all rats recorded. at the following respective doses: $24 \mathrm{nmol}, 12 \mathrm{nmol}, 6 \mathrm{nmol}, 1,5 \mathrm{nmol}$ in $1 \mu 1$. Because anorexila in TB rats in this experiment did not develop until day 26 , all rats were again tested with $12 \mathrm{nmal}$, on day 29.

\subsection{Results}

As may be observed from fig. 12.3.-1, NE stimulation in the first experiment was undertaken during significant anorexia $(t=2.86 . p<0.01)$ in TB animals, that increased across days. NE NE elicited eating was somewhat diminished in TB animals as compared to NTB control animals, without gaining significance, on days 33 through 37 . A 2-way ANOVA of NE induced food intake following methysergide or normal saline pretreatment on days 39 and

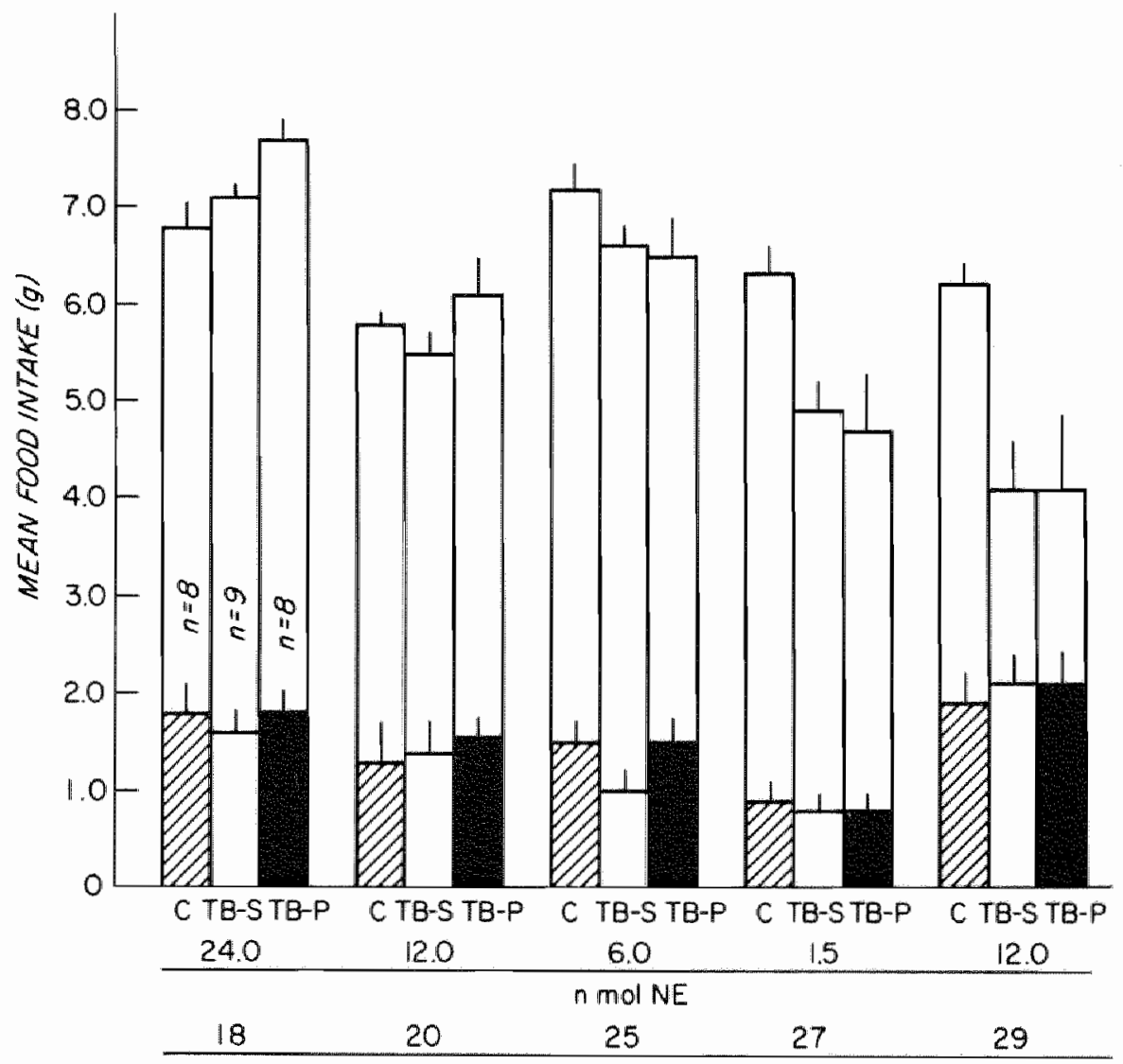

DAYS AFTER TUMOR INDUCTION

Fig. 12.3.-2 Mean ( $\$$ SEM) daily food intake ( $\mathrm{g} / 100 \mathrm{~g}$ body weight, open bars) and dose-response analysis of eating (total $g_{\text {。 }}$ insert bars) following the injection of norepinephrine (NE) into the periventricular hypothalamus of MCA sarcoma bearing (TB) and control (C) rats. Drug-stimulated eating is corrected for eating following the intrahypothalamic (ht). injection (1 $\mu$ ) of normal saline. Brain serotonin was depleted in one group of TB rats (TB-P) by the injection (ip.) of parachloroamphetamine (PCA) $(10 \mathrm{mg} / \mathrm{kg})$ one week prior to the induction of tumars. 
41 revealed significant methysergide effects for both days $(F(1.21)=5.50: p<0.05$. and $F(1,21)=14,60,0<0.04$ respectiwely). In addition, NE induced eating was significantly different as compared to Sal injected controls across groups on day $39(F(1.21)=6.16 ; p<005)$, but no group $x$ drug interaction was observed.

ANOVA of NE induced eating results in the second experiment, undertaken to investigate a dose-response relationship of $N E$ to eating in $T B$ animals, and to further inwestigate a possible serotonergic inhibition of NE induced eating through 5-HT depletion by administration of PCA, indicated no differences across groups on each test day (Fig. 12.3.-2). Analysis of $24 \mathrm{hr}$ food intake data revealed significant differences across groups from day 26 on $(F(2,20)=3.99 \%, 0.05)$. Past hac t-tests indicated diminished eating in both TB groups $(p=0.05)$, with no differences being observed between the TB groups.

\subsection{Discussion}

The results of these experiments may fit into a concept of serotonin exerting an antagonistic effect on NE induced feeding at the level of the paraventricular hypothalamus (Leibowitz, $1978^{\circ}$ ). Thus, the significant facilitation of NE induced eating on days 39 and 41 by methysergide intervention in experiment 1 may support this concept.

However, although disinhibition of NE induced eating by int administration of methysergide occurred in both TB and FF groups, no differential response by TB animals was observed, indicating that the anorexia producing mechanism(s) in TB animals remained unaffected by NE or methysergide manipulation. Although some indication of a diminished response of TB animals to NE stimulated eating existed in the first experiment, this difference could not be substantiated in the second experiment. It may be argued that at the time of the actual doseresponse investigation in the second experiment anorexia was not present, and consequently the hypothesized serotonergic inhibition not active. It should be emphasized however that later on in this experiment, when anorexia was present, no different response was observed following two different NE-doses. These results thus confirm reports that suggest the hypothalamus not being involved in the mediation of cancier anorexia (Morrison, 1968). Neither did our own results (Chapter 6 ) demonstrate altered 5 - HT metabolism in the hypothalamus of anorectic MCA TB rats.

The explanation, given in chapter 10 for the observed lack of effect of methysergide in TB animals may be valid for this experiment as well: the inhibitory serotonergic activity on food intake may be mediated primarily through the serotonin 1 receptor, probably at other levels than the hypothalamus, while the antagonistic effects of methysergide are more potent at serotonin 2 receptors (Peroutka, 1981).

Although ip. administered para-chloroamphetamine (PCA) has been described to induce selective brain 5-HT depletions (Sanders-Bush, 1977) no effects of this intervention on food intake have been described.

Results of this experiment suggest, that 5-HT depletions per se do not result in hyperphagia in TB rats, without knowing however what effects would be induced in FF rats, and therefore seem to parallel results obtained and discussed after administration of another neurotoxic drug, 5,7-dihydroxytryptamine (Chapter 11).

\subsection{Conclusions}

1. Norepinephrine induced eating is not influenced by the presence of a tumor. There may be severall reasons for this observation: no interaction taking place between hypothesized - increased 5-HT activity and NE at the level of the investigation (PVH), or 5-HT not being inwolved in the mediation of cancer anorexia. 
2. Although postsynaptic blockade of serotonergic neurons at the level of the PVH does facilitate NE induced eating. the lack of a differential response of TB animals, as compared to NTB controls, suggests again that - hypothesized - inhibitory 5-HT activity in TB rats does not occur at the level of investigation, or that 5-HT is not involved at all in the mediation of cancer anorexia.

3. Serotonin depletion by PCA administration does not facilitate NE induced feeding in TB animals, thus contributing to the uncertainty of serotonin mediating cancer anorexia. 


\section{Chapter 13}

\section{Summary of the results and conclusions of the experiments described in part III}

\subsection{Tryptophan and serotonin}

Experiments in this part were designed to manipulate the serotonin system and were described in a sequence that parallels serotonin synthesis and activity.

\subsubsection{Effort to lower brain tryptophan}

Tryptophan transport into the brain has been proposed to be serotonin synthesis-rate affecting. This transport is influenced by competition for binding to the neutral amino acid carrier by the other neutral amino acids, among them the branched chain amino acids. Diet enrichment with these compounds to a level of $37 \%$ of total casein resulted in increased plasma levels of the branched chain amino acids, but failed to alter the plasma ratio free tryptophan over the neutral amino acids in either test group. Therefore, the mechanism by which the significant delay of 2 days in the onset of cancer anorexia in Walker 256 tumor bearing animals was effected, is not certain.

In addition, further enrichment of the diet with branched chain amino acids " to $47 \%$ of total casein, did not result in further delay of cancer anorexia. This finding may result from the diet having become imbalanced, or too nitrogen-enriched, thus exerting inhibitory effects on food intake behavior. Finally, learned food aversions may have played a role to this effect.

\subsubsection{Effect of serotonin synthesis inhibition}

Inhibition of the serotonin synthesis-rate affecting enzyme, tryptophan hydroxylase, by the intraventricular administration of parachlorophenylalanine in Walker 256 tumor bearing and control animals resulted in significant depletion of brain serotonin, illustrated by significant lower brain serotonin levels, and decreased levels of its metabolite 5-hydroxyindoleacetic acid.

This depletion of central serotonin was in tumor bearing animals associated with a significant delay in the onset of cancer anorexia by two days, while no hyperphagia was observed in parachlorophenylalanine injected control animals as compared to saline injected control animals.

The presence of significant anorexia in parachlorophenylalanine injected tumor bearing animals in combination with still significantly decreased levels of the serotonin metabolite 5-hydroxyindoleacetic acid in these animals suggests additional mechanisms being active in the mediation of cancer anorexia.

\subsubsection{Effect of postsynaptic receptor blockade}

Methysergide, intraperitoneally administered, has been reported to inhibit the appetite 
suppressant activity of fenfluramine, which presumably acts through a serotonergic mechanism. Across two experiments the intraventricular administration of this serotonin blocker, up to a dose of $100 \mu \mathrm{g}$. failed to increase food intake in methylcholanthrene induced sarcoma bearing animals, in the presence of increased central serotonin acitivity in these animals.

The reasons for this fallure may be manifold: no serotonin involvement in the mediation of cancer anorexia, use of a suboptimal dose, or a too extended lood intake observation period. Finally, it was hypothesized that inhibition of food intake by serotonin is primarily transmitted via the inhibitory, serotonin 1 receptor, for which methysergide has low affinity. as compared to its affinity for serotonin 2 receptors.

\subsubsection{Effect of the neurotoxic druig 5,7-dihydroxytryptamine}

The drug 5,7-dihydroxytryptamine has been reported to inhibit tryptophan hydroxylase and to cause a degeneration of serotonergic neurons selectively, if norepinephrine neurons are protected against depletion by pretreatment with desmethylimipramine.

Although hyperphagia and body weight gain has been reported to occur following selective serotonin depletion with this drug, these observations have proven not to be reproducible. Serotonin depletion by administration of 5,7-dihydroxytryptamine did not disinhibit feeding in either control or Walker 256 tumor bearing animals, while tryptophan and 5-hydroxyindole values, and food intake data replicated our previous results.

\subsubsection{Effect of central chemical stimulation}

Norepinephrine, when injected into the perifornical hypothalamus, has been demonstrated to elicit eating. This response was not influenced by the presence of a methylcholanthrene induced sarcoma, but was facilitated by pretreatment with the serotonin receptor blocker methysergide albeit that no differentiation between tumor bearing and non-tumor bearing animals regarding this blocking effect was observed.

It was hypothesized that no interaction was taking place between the presumed increased serotonin activity and norepinephrine at the level of investigation (paraventricular hypothalamus), or that serotonin was not involved in the mediation of cancer anorexia if serotonin mediated inhibition of norepinephrine induced eating would take place at the level of the paraventricular hypothalamus, as has been postulated.

In addition, parachloroamphetamine induced serotonin depletion did not facilitate the norepinephrine induced feeding in methylcholanthrene induced sarcoma bearing rats, thus contributing to the uncertainty of serotonin as a mediator of cancer anorexia.

\subsubsection{Conclusion}

Results of experiments described in this part do not show a consistent support for the hypothesized serotonergic mediation of cancer anorexia. Thus, although - presumed inhibition of serotonin synthesis seemed to postpone the onset of anorexia, blocking serotonergic receptors, destroying serotonergic neurons and chemical stimulation of the paraventricular hypothalamus in combination with manipulations of the serotonin system, did not result in restoration of food intake in tumor bearing animals to normal levels, or a differential response to chemical stimulation in tumor bearing animals, respectively. 


\subsection{Catecholamines}

In some experiments brain levels of norepinephrine and dopamine were determined. No changes were observed in dopamine levels on any occasion. Norepinephrine levels were reduced in Walker 256 tumor bearing animals, that were treated with parachlorophenylalanine. This observation may account for the delay in the onset of cancer anorexia by the above treatment.

In two other experiments (chapter 10 an 11) no consistent pattern of norepinephrine changes could be observed, thus making interpretation of a possible role for this neurotransmitter in the mediation of cancer anorexia wery difficult. 
Part IV

Concluding reflections 


\section{Chapter 14}

\section{Cancer anorexia}

Experiments in this thesis have focused the influence of amino acids, and more specifically of tryptophan, on the neuroregulation of food intake in tumor bearing animals. The hypothesis that was tested concerns altered peripheral TRP metabolism, inducing increased brain TRP levels, followed in turn by increased central $5-H T$ synthesis and degradation. Associated with reports from literature indicating 5-HT as a mediator of satiety it was hypothesized that cancer anorexia may be mediated by this increased central serotonergic activity.

\subsection{Plasma tryptophan and cancer anorexia}

Although the study of peripheral TRP alterations has not been the main purpose of these experiments, the observed fairly consistent changes need further reflection. Tryptophan is an essential amino acid to most mammals, including the rat, distributed in serum between an unbound pool, and a fraction that is bound to albumin (Chapter 1, section 1.4.). Thus, plasma TRP concentrations are dependent on intake of this amino acid in the diet. At the other end plasma TRP concentrations are determined by degradation, primarily by two pathways. One involves hydroxylation to 5-hydroxytryptophan by tryptophan hydroxylase, primarily present in the brain, followed by decarboxylation to serotonin by 5-hydroxytryptophan decarboxylase, also present in the brain, but also in the kidney. The other pathway involwes oxidation to kynurenine by 2,3-dioxygenase, primarilly present in the liver. Activation of this enzyme is induced by TRP or adrenal corticosteroids. Madras (1974) found liver concentrations of TRP unaltered by diet induced fluctuations in the serum unbound pool, nor was 2,3-dioxygenase activity altered. However, administration of salicylates caused TRP displacement from albumin, followed by increased 2,3-dioxygenase activity. Chronic use of this drug induced decreased plasma total TRP levels, thus suggesting that chronic elevations of the plasma unbound fraction may induce decreasing plasma total TRP concentrations, possibly by activation of 2,3-dioxygenase in the liver. Insulin administration increases TRP concentrations in rat plasma, in contrast to the lowering effect on all other amino acids (Fernstrom, 1972 $2^{\text {b) }}$. Glucagon administration however reduces plasma TRP levels (Fernstrom, 1972 ). Whether this effect is the result of the lipolytic state that is associated with elevated glucagon levels, thus inducing higher plasma unbound TAP fractions, is not known. Thus far the observed lower plasma total TRP levels in tumor bearing animals have been ascribed to decreased dietary intake. Recently however, $v$. Lammeren (1982) published results of farced feeding experiments, in which Walker 256 tumor bearing animals received $125 \%$ of $24 \mathrm{hr}$ food intake by matched FF control animals. These results indicated that plasma total TRP concentrations increased to the levels of total TRP in pair-fed animals, that were, however, still significantly lower than the concentrations in FF animals, while plasma free TRP levels in forced fed TB animals remained $200 \%$ of normal values. These results may suggest that in TB animals, in addition to decreased intake, increased TRP degradation is responsible for the observed lowered plasma tolal TRP concentration. This phenomenon, in turn, may be induced by increased 
glucagon levels that we recently described ( $v$. Lammeren, 19829$)$ in $W_{256}$ TB animals, and that have been reported to reduce plasma total TRP concentrations (Fernstrom, $1972^{\circ}$ ). The plasma free TRP traction being continually elevated in forced fed TB rats may also be explained by these glucagon concentration increments. Thus, elevated glucagon levels would be responsible for the lipolytic state, accompanied by increased plasma FFA levels. that is generally associated with cancer disease (Bodansky, 1975).

The above summarized observations of altered peripheral TRP metabolism may not be limited to cancer disease. Thus, decreased albumin and increased FFA levels, in combination with a relative insulin resistance, and elevated glucagon levels, are observations also made under circumstances of metabolic stress, e.g. sepsis, trauma etc. Little is known about total and free TRP levels under these conditions, and especially if these conditions have a protracted course. Therefore, the observed changes in TRP metabolism may not be a tumor specific phenomenon, but rather an aspecific expression of metabolic stress, as for example induced by necrosis in the tumor, or by sepsis, known to have a high incidence in cancer patients (Dionigï, 1980). The lack of knowledge regarding the regulation of free amino acid levels prohibits further characterization of the observed TAP-metabolism changes in experimental cancer.

\subsection{Brain serotonin and cancer anorexia}

Although brain 5-HT activity correlated well with cancer anorexia in these animal studies (Chapter 7), no consistent evidence in support of a causal relationship between the two observations could be obtained (Chapter 13)

A resolution to these seemingly contradictory conclusions may lie in one of the following explanations.

\subsubsection{Serotonin receptors}

Serotonin is involved in the mediation of cancer anorexia but it is only the serotonin 1 receptor that contributes to this phenomenon. Thus, the increased serotonergic actiwity suggested by the results in part II of this thesis would be mediated through serotonin 1 receptors; the facilitating effect of serotonin synthesis inhibition by branched chain amino acids or parachlorophenylalanine on food intake would not disturb this concept. That NE induced eating responses in our experiments were not different in TB animals as compared to non tumor bearing controls, can only be explained by the assumption that NE and the anorexia causing mechanism do not interact at the level of the paraventricular hypothalamus.

It leads to rejection of the hypothesis under investigation that methysergide did facilitate NE induced eating, thus confirming reports by Mabry (1973) and Leibowitz (19789) of serotonergic inhibition of NE systems in the regulation of food intake, but also suggesting that cancer anorexia is not being transmitted by serotonin, at least not at the level of the paraventricular hypothalamus.

However, in itself this contradiction would not have to interfere with the assumption, that the inhibitory effects on food intake would be transmitted only through the serotonin 1 receptor, because little is known about the anatomical distribution of serotonin 1 and 2 receptors. In addition to the possibility of only one type serotonin receptor trans-mitting inhibition signals, alterations in serotonin receptor binding under influence of a tumor should be considered. This alteration may result in increased affinity of the receptor for serotonin, irrespective of involvement of either one of the 2 receptors or both. Samanin (1980 atb) and Fuller (1980) reported different binding properties to serotonin receptor sites of 5-HT 
agonists, such as quipazine, and metachlorophenylpiperazine. Furthermore, Enna (1981) described altered receptor binding in different neuropsychiatric disorders. Thus, according to this concept of altered serotonin receptor binding, serotonin synthesis inhibition would facilitate eating by whole brain serotonin depletion, while serotonin antagonists and neurotoxins would not act at the sites that are of primary importance in the transmission of feeding inhibition through serotonergic neurons. Such a concept could explain the results im part II and part III of this thesis. Contradictory to this concept. however, would be the modest serotonin depletions, obtained with branched chain amino acid enriched diets and p-CPA, having an effect on food intake, and a far better depletion of whole brain serotonin presumably obtained with for example PCA, which does not disinhibit feeding.

Finally, transmitter synthesis rates may be different relative to precursor supply in different brain regions, as has been suggested in Chapter 6, and by Knott (1974) and Curzon (1980). The lack of effect of serotonin antagonists, such as methysergide, and neurotoxins, such as PCA, should then be explained by a failure of these drugs to act at the sites of increased serotonin synthesis.

\subsubsection{False neurotransmitter formation}

Another product of TRP metabolism, tryptamine, may mediate cancer anorexia. The increased amounts of brain TRP would, according to this hypothesis be decarboxylated to tryptamine in both catecholaminergic and serotonergic neurons (Young. 1980). The synaptic activities of this putative neurotransmitter would not be affected by antagonists and meurotoxic drugs that act on 5-hydraxytryptaminergic neurons. Both the results in part II and part $1 \| l$ could be explained by this hypothesis. On the other hand, synthesis of tryptamine is known to occur in a very small portion of circulating TRP under normal circumstances.

\subsubsection{No specific serotonin involvement}

Another interpretation of the observations may be that serotonin is not specifically involved The increased serotonergic activity, reported in part 11, would be a consequence of undernutrition (Perez-Cruet, 1972; Tagliamonte, 1973; Curzon, 1975; Young, 1976), not present in pair-fed animals, because these animals are not deprived enough "as compared to tumor bearing animals, that are under the additional metabolic stress induced by the presence of the tumor. Subsequently, this increased serotonergic activity induced by the sewerely deprived TB animals, would mediate a general inhibitory control of feeding behavior, on top of which other systems would influence specifically eating and drinking. Thus, results in part II would support this concept, while the feeding facilitation obtained by administration of branched chain amino acids and P-CPA being limited to 48 hrs could be explained by the assumption that mechanisms specifically modulating eating and drinking become of overriding importance, and thus induce anorexia. The lack of such a transient effect of methysergide and 5,7-dihydroxytryptamine should in this respect be explained with the same arguments discussed in chapters 10 and 11 respectively. Finally, the apparently normal function of the paraventricular hypothalamus in TB animals that was described in chapter 12 , does not disturb the above hypothesis.

In summary, the observed increased serotonergic activity may be non specific in the sense, that this system exerts inhibitory control over a large variety of motivated behaviors. Thus, it inhibits waking, self stimulation, sexual behavior and aggressiveness' (Panksepp, 1979). 


\subsubsection{Conclusion}

Although each one of the above mechanisms may be active, and responsible for the diverse responses to manipulations of the 5-HT system, and for that reason require further investigation, the possibility that serotonin mediates a general inhibitory control over motivated behaviors, including feeding, on top of which other systems specifically modulate eating and drinking. should be considered in the genesis of cancer anorexia.

\subsection{Tyrosine, catecholamines and cancer anorexia}

Tyrosine is a nonessential amino acid, that is synthetized by a one-step reaction, hydroxylation, from the essential amino acid phenylalanine. Thus, if tyrosine is fed, the diettary requirement for phenylalanine is reduced substantially.

Tyrosine, and phenylalanine, are neutral amino acids that compete for the same NAA-carrier in the blood-brain barrier. Furthermore, the Michaelis-Menten constant of tyrosine hydroxylase is high relative to brain concentrations of this $A A$, suggesting that brain tyrosine concentration constitutes an additional factor controlling catecholamine synthesis. Thus, Wurtman (1974) hypothesized that the administration of any NAA other than tyrosine, or phenylalanine, may be expected to suppress catecholamine symthesis, and consequently that dietary intake of phenylalanine and tyrosine may be expected to influence central catechol synthesis, thereby parallelling mechanisms in the TRP - 5-HT system. Krause (1979 ${ }^{6}$ ) described increased tyrosine levels in plasma and brain in both $W_{256}$ tumor bearing and pair-fed animals. Because pair-fed animals showed even higher tyrosine levells than TB rats did, this phenomenon was assumed to be a result of malnutrition, and an indicator that NE and DA were probably not involved in the mediation of cancer anorexia. However, based on his observations of diet induced alterations of brain tyrosine and phenylalanine concentrations Wurtman (1974) hypothesized that norepinephrine and dopamine-containing brain neurons may participate in the mechanisms by which the brain monitors the plasma amino acid pattern and the nutritional state.

\subsubsection{Norepinephrine}

Suggestive for NE involvement in the processes regulating feeding are reports of vigorous eating being ellicited by direct intrahypothalamic administration of NE (Grossman, 1962; Leibowitz, $\left.1978^{a+b}\right)$. This effect can selectively be blocked by the central administration of a-adrenergic blockers (Leibowitz, 1976).

Other lines of evidence indlicate however, that NE may also mediate satiety signals (Margulles, 1972). Leibowitz (1976) raised the possibility of anatomically distinct a-adrenergic feeding and $\beta$-adrenergic satiety systerns.

Our observations regarding brain NE levels were inconclusive. Thus, no changes correlating with cancer anorexia were seen in experiments described in part II, while on two different occasions in part III NE levels in TB amimals changed in opposite directions.

The observation by Ahlskog (1975), who saw hyperhagia accompanied by a $\pm 95 \%$ depletion of forebrain NE 'argues against a specific role of NE in the elicitation of feeding and may' indicate that (NE systems) are nonspecific in the sense that they represent some aspect of general arousal' (Panksepp, 1979). 


\subsubsection{Dopamine}

Brain dopamine (DA) activity also seems to promote feeding. Thus, lesions of the nigrostriatal DA systems produce feeding deficits, that are similar to those of lateral hypothalamic lesions (Ungerstedt, 1971). Furthermore. Heffner (1980) observed feeding in food deprived rats accompanied by increased production of the dopamine metabolite 3,4-dihydroxyphenylacetic acid (DOPAC) in the brain. This neurochemical change occurred in the nucleus accumbens, the posterior hypothalamus and the amygdala, but not in other dopaminergic nerve terminal fields. Dopamine levels were not changed in any of the groups in any brain area. This observation may illustrate that whole brain DA levels not being changed in any of the experiments in this thesis do not necessarily exclude this neurotransmitter from playing a specific role in the mediation of cancer anorexia. Indeed most recently $v$. Lammeren $\left(1982^{\circ}\right)$ reported differentially affected whole brain concentrations of the DA metabolite 3-methoxytyramine (3-MT) by tumor and pairfeeding treatments. Furthermore, the absence of reversal of changes in both 3-MT and homovanillic acid levels by an enterall forced feeding schedule suggests that alterations in brain concentrations of these DA metabolites are not due to the effects of undernutrition. Indeed. although it has been suggested that "feeding deficits following DA system lesions are largely the result of a global impairment of the capacity to generate goal directed behavior,.... there may be some specificity at the terminal fields of DA systems, .... or there is the possibility that DA systems receive imputs from specific regulatory networks' (Panksepp, 1979).

\subsubsection{Conclusion}

Although NE systems seem to play a non specific role in the control of feeding behavior, recent publications seem to indicate that DA systems may mediate specific effects on feeding and drinking. In addition, the most recent publications in the field of food intake control in cancer anorexia indicate that DA may also play a specific role in the control of feeding and drinking in this condition. Thus, DA may be (one of) the system(s) that specifically modulate(s) eating and drinking, on top of an activated general inhibitory control on this behavior mediated by serotonin. 


\section{Chapter 15}

\section{Cancer anorexia and cachexia}

Rapid weight loss, cachexia, in patients with cancer disease is due to negative energy and nitrogen balances (Lundholm, 1981). The word cachexia describes a conception that summarizes the results of several metabolic events. Thus, the presence of a growing mass may induce reactions beyond the normal range of adaptation to various kinds of stress in the host "making cancer cachexia a unique entity (Karlberg, 1980). In contrast to Karlbergs opinion, Lundholm (1981) concluded that cancer cachexia metabolism is not unique in any known fashion, being a result of anorexia and the metabolic demand of the exponentially growing tumor. This substrate demand of tumor cells was not beyond that of comparable non malignant immunereactive cells, activated by ip. injection of bacterial antigens. This demand being chronic, and becoming of quantitative importance due to the mass of the tumor, may foster the "uniqueness' of cancer cachexia metabollism.

In summary cancer cachexia is different from the cachexia, that accompanies clinical

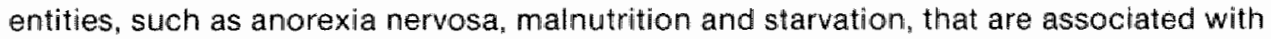
decreased intake and malabsorption of nutrients. It may show resemblance to such entities as trauma and sepsis, associated with increased energy expenditure in host organisms. The observed increased energy expenditure seems to be to a great extent the result of increased glucose turn-over, partly via increased activity of futile "cycles', such as the Cori-cycle and glucose-alanine cycle (Edström, 1980).

The above consideration, together with the observation that glycogen stores in liver tissue from tumor bearing mice were profoundly decreased, which was not found in pait-fed mice (Lundholm, 1980) may be linked to the increased glucagon levels, which we measured in $W_{256}$ TB rats. Thus, antibady-levels specific for the $\mathrm{C}$ - and the $\mathbb{N}$-terminal regions of glucagon ( $315 \pm 110$ and $538 \pm 85 \mathrm{pg} / \mathrm{ml}$ respectively) in TB animals were greater than these levels in PF (92 \pm 20 and $284 \pm 35 \mathrm{pg} / \mathrm{ml})$ and FF (136 \pm 11 and $360 \pm 23)$ control animals. while insulin levels remained unchanged ( $v$. Lammeren, 1982 ).

Furthermore, the increased energy expenditure has also been described to result from energy costs for protein synthesis in the tumor and for increased hepatic protein synthesis in the tumor host (Ekman, 1981). To which extent increased energy expenditure is tumor induced, or increased relative to the decreased intake of nutrients, is not known. Therefore. Morrison (1976 ) stating that the central problem in the cancer anorexia-cachexia syndrome is the incapability of the tumor host to adapt energy intake to the nutrient needs of host and tumor, is probably too simple a conception. The disappointing results of neutralizing decreased nitrogen and energy intake by the use of total parenteral nutrition (TPN) (Brennan, 1981) may illustrate the above.

Until recently there have been few efforts to quantitate to what extent on one hand decreased food intake, anorexia, and on the other hand altered, tumor induced increased, energy expenditure contributes to this state.

It may be important to discern these entities, because it may help to define the expectations. one may have of efforts to neutralize mechanisms causing cancer anorexia, and of interventions aimed at normalization of metabolic processes in the host.

Thus, Karlberg (1982) recently described decreased protein synthesis in skeletal muscle in MCA tumor bearing rats as a result of anorexia, whereas the increased muscle catabolism 
appears to be associated with the umor itself. Furthermore, it was observed by the same group (Lindmark, 1982) that with the onsel of anorexia the metabolic rate was significantly decreased in TB animals, resulting in decreased amino acid and glucose oxidation, whereas whole body fat oxidation was elevated, resulting in a negative energy balance.

Considering time relationships between the onset of altered host metabolism, and the onsel of anorexia in the course of tumor growth, may contribute to the understanding of the importance of each factor relative to the development of cachexia. Most likely to occur is that the development of anorexia is preceded by increased energy expenditure, initiated by alterations in the metabolic pathways in the tumor host.

Indeed. Pratt and Putney reported already in 1958 increased energy expenditure occurring right after inoculation of Walker 256 tumors in rats, well betore tumors became palpable. Also, intrinsic to the hypothesis investigated in this thesis, is that anorexia caused by increased CNS - 5-HT activity is preceded by changes that are considered parameters of cancer cachexia, e.g. lowered plasma albumin levels and increased lipolysis, resulting in increased plasma free fatty acid concentrations.

Furthermore, alterations in liver composition, limited to TB animals, though determined with techniques of limited sophistication (Chapter 3), seemed to precede the development of anorexia.

However, the clinical importance of anorexia and therefore the ultimate goal of research efforts, is lying in the notion, that restoration of normal (central) control mechanisms of food intake, will not only maintain energy and nitrogen intake on "normal" (pre-disease) levels, as was the reference standard for definition of anorexia in this thesis, but also adapt intake to expenditure, thus expelling any additional claim from the part of decreased intake on the development of the cachexia syndrome in cancer disease. 


\section{Summary}

Cancer anorexia is a common and oftem believed early phenomenon in cancer disease. The incidence of anorexia is not known, and no quantification of the problem has been attempted, partly because of difficulty to isolate this phenomenon in the multiplicity of events that take place once a cancer is growing, and partly because normal physiology of hunger and satiety is not well understood.

In chapter 1 some of the theories proposed for the regulation of food intake, and their possible role in the mediation of cancer anorexia, are reviewed. Among others the influence of amino acids on the neuroregulation of food intake is reviewed. Some years ago it became under consideration that the brain is susceptible to dietary influences and consequently that diet induced changes of plasma amino acid levels will result in alterations of brain concentrations of these amino acids. The essential amino acid tryptophan has been widely studied because it is the precursor amino acid for synthesis in the brain of the neurotransmitter serotonin, that has been associated with inhibitory control on food intake behavior. Therefore, factors influencing tryptophan transport into the brain are reviewed. In relation to cancer Krause et al. described increased plasma free tryptophan concentratians in tumor bearing rats, as compared to semistarved and normal control animals. This alteration was found to be a result of decreased tryptophan binding to albumin because of decreased albumin concentrations, and increased concentrations of the for binding competing free fatty acids. He subsequently indicated that these increased plasma free tryptophan levels were in the presence of normal blood-brain barrier amino acid carrier activity probably responsible for the concomitantly observed increased brain levels of tryptophan, and of the serotonin degradation product 5-hydroxyindoleacetic acid. By association of these indications for increased central serotonergic activity with reports from literature that suggest serotonin to mediate satiety, Krause hypothesized that increased central serotonergic activity, induced by peripheral tryptophan binding alterations, could mediate the anorexia, observed in experimental cancer.

The purpose of the studies in this thesis is to test the functional validity of this hypothesis. To achieve this, two types of experiments are used: on one hand correlations, proposed by Krause between cancer anorexia and altered biochemistry are further detailed (chapters $3,4,5,6$, summarized in chapter 7 ). On the other hand manipulations of the central sertotonin system are performed and their effects on food intake behavior in cancer bearing rats evaluated (chapters $8,9,10,11,12$, summarized in chapter 13).

In chapter 2 experimental designs, the animal-tumor models, and the biochemical and statistical techniques used in the thesis are described.

Chapter 3 describes 4 experiments undiertaken to confirm earlier data, and to further define the Walker 256 carcino sarcoma model, that was used in the earlier experiments. Results show that implantation of a Walker 256 tumor in immature female Sprague-Dawley rats will kill the tumor host, will induce anorexia on a constant moment in the course of tumor growth, but will not induce the emaciation, generally associated with cancer disease, while tumor progression is limited to the stage of regional disease. Biochemical analyses show, compared with semistarved and normal control animals, decreased plasma total tryptophan levels in tumor bearing rats, and increased free tryptophan levels, which may, in the 
presence of normal blood-brain barrier neutral amino acid carrier activity, be responsible for increased brain TAP levels, that in turn may cause the higher levels of brain serotonin and 5 hydroxyindoleacetic acid, suggestive for increased serotonin tumover.

The experiments in chapter 4 investigate the time relationship between the development of cancer anorexia and the onset of biochemical changes. These experiments were performed in the Walker 256 tumor model, because the onset of anorexia is predictable in this model Results show changes in central nervous system serotonin activity occurring immediately prior to the onset of cancer anorexia. Catecholamine levels did not show any consistent correlation with the onset or severity of cancer anorexia.

The experiments in chapter 5 were performed in a different tumor model. A methyleholanthrene induced sarcoma implanted in adult male Fisher 344 rats induces prolonged anorexia, and significant cachexia in tumor bearing animals while growth in pairfed control animals is not sustained. The absence of such changes in the undernourished pair-fed animals, and the similarity of the tryptophan and serotonin metabolism changes with the Walker 256 tumor bearing animals suggest that the biochemical alterations in the serotonin system in these tumor bearing rats have a causative relationship to the development of cancer anorexia, rather than being a consequence of decreased food intake. Furthermore, the biochemical changes seem to be a general phenomenon, demonstrated in two tumor lines. Finally, the increased dopamine and norepinephrine levels relative to the values measured in pair-fed animals may suggest a catecholaminergic involvement in the mediation of cancer anorexia in addition to the hypothesized role of serotonin.

Chapter 6 reports results of analyses of regional brain serotonin activity in tumor induced anorexia in both tumor models. These studies seem to indicate that serotonin activity in anorectic tumor bearing rats is elevated in gross reglons, that include areas that have been implicated in the control of food intake, although the functional relationship of regional changes in serotonin metabolism to feeding behavior is uncertain.

In chapter 7 results of the above described experiments are summarized. It was concluded that the Walker 256 tumor model in immature female Sprague-Dawley rats is a highly reproducible model of cancer anorexia, so acute that onlly mild cachexia will develop before the tumor kills the host, and that the methylcholanthrene induced sarcoma in adult male Fisher 344 rats is a model of prolonged anorexia and severe cachexia. By association of the observed suggestions for increased central serotonergic activity in both tumor models, with the published claims of serotonin mediation of satiety, it is hypothesized that cancer anorexia may be mediated, at least in part, by serotonin. Results of the determinations of catecholamines should be followed by a more systematic investigation into a possible role for these neurotransmitters in the mediation of cancer anorexia.

As tryptophan shares a common transport carrier with the other neutral amino acids in the blood-brain barrier, transport of this amino acid is susceptible to competition by these neutral amino acids. Increasing plasma levels of the branched chain amino acids, accomplished by diet enrichment with these amino acids, in order to obtain increased competition was the purpose of experiments described in chapter 8 . Feeding a $37 \%$ branched chain amino acid diet to Walker 256 carcino sarcoma bearing animals results in a 48-hour delay in the onset of cancer anorexia, as compared to control animals, fed a 25\% branched chain amino acid, isonitrogenous control diet. Further enrichment of the diet with these compounds does not show such a behavioral effect. The mechanisms behind these observations are not known.

Inhibition of the key enzyme in serotonin synthesis, tryptophan hydroxylase, is achieved by central administration of parachlorophenylalamine. Intraventricular injection of this drug in tumor-bearing animals (chapter 9) results in significant depletion of brain serotonin associated with a significant delay in the onset of cancer anorexia by two days. The tumor bearing animals eventually becoming anorectic in the presence of continued lower 5-hydroxyindoleacetic acid levels, suggests an aspecificity of serotonin in the mediation of 
cancer anorexia ${ }$ and additional mechanisms being active.

Intraventricular administration of the serotonin receptor blocker methysergide in different doses failed to increase food intake in tumor bearing animals, though increased central serotonin activity was suggested in these animals (chapter 10). It was hypothesized, that cancer anorexia could be mediated primarily through serotonin 1 receptors, because methysergide shows a higher affinity tor serotonin 2 receptors.

Selective serotonin depletion by central administration of the serotonin neurotoxic drug 5,7-dihydroxytryptamine does not disinhibit feeding in either control or tumor bearing animals (chapter 11). Although administration of this drug has once been reported to result in increased food intake and body weight gain, subsequent studies have not been able to replicate these results. Thus, the significance of the absence of a response to serotonin depletion by this drug in cancer anorexia is uncertain.

The response to chemical stimulation of the paraventricular hypothalamus in tumor bearing animals was tested in two experiments described in chapter 12. It was concluded that norepinephrine induced eating is not influenced by the presence of a tumor and that mamipulation of the serotonin system at the level of investigation, viz. the paraventricular hypothalamus, does not alter differentially the response to this stimulation in tumor bearing animals. Thus, these results again contribute to the uncertainty of serotonin mediating cancer anorexia.

Chapter 13 summarizes the results of the previous experiments and concludes that manipulation of the serotonin system does not lead to consistent support for the hypothesized serotonergic mediation of cancer anorexia. Furthermore, determination of norepinephrine and dopamine levels did inot indicate catecholaminergic involvement in the mediation of cancer anorexia.

Chapter 14 discusses the mechanisms that may cause the observed indications for altered tryptophan metabolism, and concludes that further research concentrating on factors that regulate tryptophan plasma levels, should be conducted before any specificity of the observed changes in relation to cancer disease can be claimed. Furthermore, the seemingly contradictory conclusions of the two sets of experiments presented in this thesis regarding the hypothesized serotonergic role in cancer anorexia is discussed. The general conclusion is that serotonin may mediate a general inhibitory control over motivated behavior, including feeding. On top of this phenomenon other mechanisms may mediate specifically cancer anorexia. Most recent research indicates that the dopaminergic system may be one of such mechanisms.

Chapter 15 reviews very briefly to what extent cancer anorexia contributes to the development of cancer cachexia , the state of emaciation in cancer disease that contributes significantly to the morbidity and mortality of this disease. It is concluded that little is known about this specific quantification, but also that adaptation to energy expenditure, and thus restoration of normal regulatory mechanisms, will expell any additional claim from the part of decreased intake on the development of cachexia in cancer disease. 


\section{Samenvatting}

Anorexie, het verlies van eetlust is een frequent voorkomend symptoom bij kanker, dat, naar algemeen aangenomen wordt, vaak in een vroeg stadium van de ziekte optreedt.

De incidentie van anorexie is onbekend, en tot nu toe werden geen pogingen ondernomen om het probleem te quantificeren, zowel voor wat betreft de incidentie, als voor wat betreft de bijdrage van anorexie in het ontstaan van cachexie. Met name dit laatste lenomeen is bij kanker van significant belang voor het ontstaan van morbiditeit en mortaliteit. Indirect wordt daarmee de noodzaak onderstreept om kennis te verkrijgen omtrent de pathofysiologie van kanker anorexie. Het gebrek aan deze kennis is enerzijds te wijten aan de moeilijkheid om de anorexie te isoleren uit de veelheid van veranderingen, die optreden onder invloed van een maligniteit, en anderzijds aan het feit, dat de fysiologie van eetgedrag, d.w.z. van honger en verzadiging, niet goed bekend is

In hoofdstuk 1 wordlen enkele theoriën beschouwd die opgesteld werden met betrekking tot de regulering van eetgedrag en hun mogelijke rol in het ontstaan van kanker anorexie. Onder andere wordt de rol van aminozuren in de regulering van eetgedrag dooir neuronale structuren bezien. Ten aanzien van dit systeem kwam men enkele jaren geleden tot het inzicht dat de hersenen miet autonoom voedingsstoffen uit de bloedbaan kunnen opnemen, ongeacht de voedingstoestand van het individu, maar dat het milieu achter de bloed-hersen barrière ook beinvloedbaar is door veranderingen in het dieet. Derhalve zullen door het dieet geinduceerde veranderingen van plasma-aminozuur concentraties resulteren in veranderingen van de concientratie van deze aminozuren in het hersenmilieu. Het essentiële aminozuur tryptophaan is in dit opzicht uitgebreid bestudeerd, omdat dit aminozuur in de hersenen de voorloper is van de synthese van de neurotransmitter serotonine. Deze neurotransmitter is geassocieerd met een remmende werking op eetgedrag. In dit hoofdstuk wordt daarom een kort resumé gegeven van de factoren die het bloed-hiersen barrière transport van tryptophaan beinwloeden. Met betrekking tot kanker hebben Krause en medewerkers een verhoogde concentratie van het ongebonden tryptophaan in het plasma wan tumordragende ratten in vergelijking met ondervoede en normale controle ratten beschreven. Deze afwijking werd toegeschreven aan verminderde tryptophaan binding aan albumine, omdat de albumine concentraties bij deze dieren verlaagd waren en de voor binding competerende vrije vetzuren concentraties verhoogd bleken. In de aanwezigheld van een normale activiteit van het aminozuur transport systeem in de bloed-hersen barriere. duidde hij deze plasma-afwijkingen als voornaamste veroorzaker van de tegelijkertijd in de hersenen gevonden verhoogde tryptophaan concentraties bij tumordragende ratten. Deze verhoogde concentratie van tryptophaan in de hersenen zou aanleiding geven tot verhoogde serotonine synthese en degradatie. Door deze biochemische afwijkingen te correleren aan de van serotonine bekende inhibitie van eetgedrag, kwamen Krause en medewerkers tot de hypothese, dat verhoogde centrale serotoninerge activiteit gebaseerd op veranderingen in de tryptophaan binding in het plasma, een oorzakelijke rol zou kunnen spelen in het ontstaan van kanker anorexie bij proefdieren. Het doell van de studies in dit proefschrift is de functionele waarde van bovengenoemde hypothese te testen. Om dit te kunnen doen werden twee soorten experimenten gebruikt; in de ene groep experimenten werden de correlaties tussen kanker anorexia en veranderde serotonine biochemie, waarvan krause het 
bestaan suggerearde, werder uitgeverk (hooldstuk $3,4,5,6$, samengevat in hoofdstuk 7 ), terwill in de andere groep het serotoninerge systeem op werschillende wijzen werd gemamipuleerd, waarbij hel effect van deze manipulaties op eetgedrag bij kankerdragende ratten werd geevalueerd (hoofdstukken $8,9,10,11,12$, samengevat in hoofdstuk 13).

In hoofdstuk 2 worden de opzet yan de experimenten, en de gebruikte tumor-modellen bij de rat beschreven, alsmede de biochemische en statistische technieken die voor de studies in dit proeischrift werden aangewend.

Hoofdstuk 3 beschrifft wer experimenten, die werden ondemomen om de resultaten van Krause te bevestigen, en het Walker 256 carcinosarcoma tumormodel bij de rat, dat voor deze oorspronkelijke studies werd gebruikt, te definièren.

De resultaten van deze proeven toonden aan dat implantatie van de Walker 256 tumor in onvolwassen vrouwelijke Sprague-Dawley ratten de tumor-gastheer zal doden, anorexie zal induceren op een constant moment in het tumorgroeimproces maar niet zal leiden tot de ernstige vermagering die in het algemeen geassocieerd wordt met kanker. De tumorgroei bilift beperkt tot een regionaal proces. De biochemische analyses toonden aan dat bij tumardragende ratten vergeleken met ondervoede en normale controle dieren de plasma totaal tryptophaan concentraties verlaagd en plasma ongebonden tryptophaan concentraties werhoogd waren. Deze afwijkingen zouden bij normale aminozuur transport activiteit in de bloed-hersen barrière verantwoordelijk kunnen zijn voor de tegelijkertijd vastgestelde werhoogde hersen tryptophaan concentraties die geassocieerd bleken met verhoogde hersen serotonine concentraties en verhoogde concentraties van de serotonine metaboliet 5-hydroxyindolazijnzuur.

De experimenten in hooldstuk 4 onderzoeken de relatie in tijd tussen het ontstaan van kanker anorexie en het ontstaan van de biochemische verandering in het serotonine systeem. Deze experimenten werden gedaan in het Walker 256 tumor model, omdat bij dit model het ontstaan van anorexie met vrij grote zekerheid voorspelbaar is. De resultaten tonen aan dat de verandering van serotoninerge activiteit in de hersenen onmiddellijk voorafgaat aan het ontstaan van kanker anorexie. Catecholamine concentraties in de hersenen vertoonden geen enkele correlatie met het ontstaan of de ernst van de kanker anorexie.

De experimenten in hoofdstuk 5 werden in een ander tumormodel gedaan. Een methylcholanthreen geinduceerd sarcoma, dat geimplanteerd werd in volwassen mannelijke Fisher 344 ratten, veroorzaakte langdurige anorexie en een significante cachexie bij de tumordragende dieren, terwijl de normale groei bij gepaard gevoede controle dieren ongedaan werd gemaakt. Ongewijzigd blijven van het serotonine metabolisme bij deze ondervoede niet kankerdragende ratten en de gelijkenis van de afwijkingen in het tryptophaan en serotonine metabolisme met de waarnemingen bij het Walker 256 tumormodel suggereren dat de biochemische veranderingen in het serotoninerge systeem een causale relatie hebben met de ontwikkeling van kanker anorexie, en niet het gevolg zijn van de ondervoeding die bij dit ziektebeeld wordt giezien. Voorts lijkt de conclusie gerechtvaardigd dat deze biochemische veranderingen een algemeen fenomeen zijn en niet gebonden aan een bepaald tumormodel. Tenslotte werden bij dit tumormodel voor het eerst afwijkingen in het catecholaminerge systeem gemeten die op een additionele rol in het ontstaan van kanker anorexie van deze neurotransmitters zouden kunnen wijzen.

Hoofdstuk 6 geeft de resultaten van de analyse van serotoninerge activiteit in verschillende hersendelen weer, zoals die werd uitgevoerd bij beide tumormodellen. Deze studies suggereren dat serotoninerge activiteit bij anorectische tumordragende ratten verhoogd is in gebieden die kernen bevatten welke in de literatuur geassocieerd zijn met de controle over voedselinname gedrag. Er is echter weing bekend over de functionele relatie tussen parameters van regionale serotoninerge activiteit en eetgedrag.

In hooldstuk 7 worden de resultaten van de experimenten uit de vorige hoofdstukken samengevat; ten aanzien van de tumormodellen wordt geconcludeerd dat het Walker 256 
tumormodel in onvolwassen wrouwelijke Sprague-Dawley ratten een goed reproduceerbaar model voor kanker anorexie is dat zo snel tol de dood van de gastheer leidt, dat slechts een milde cachexie ontstaat en dat het methylcholanthreen geinduceerde sarcoma geimplanteerd in volwassen mannelijke fisher 344 ratten een model voor langdurige anorexie en ernstige cachexie is. Door associatie van de gevonden suggesties voor het bestaan van verhoogde cemtrale serotoninerge activiteit bij beide tumormodellen met de bewering uit de literatuur dat serotonine verzadiging zou overbrengen, wordt de hypothese bevestigd dat kanker anorexie, op zijn minst gedeeltelijk, door serotonine veroorzaakt zou kunnen worden. Voorts zou een mogelijke catecholaminerge rol in het ontstaan van kanker anorexie nader onderzocht dienen te worden op basis wan de afwijkingen, gevonden in de hiervoor beschreven experimenten.

Omdat tryptophaan woor transport naar de hersenen door de bloed hersen barriere een aminozuur transport systeem gebruikt dat dit aminozuur deelt met andere neutrale aminozuren, is dit transport gevoelig woor competitie van deze neutrale aminozuren. Door de plasma concentraties van de vertakte keten aminozuren te verhogen, hetgeen bereikt werd door het rattenvoer te verrijken met vertakte keten aminozuren, zou de competitie voor binding aan het aminozuur transport systeem vergroot kunnen worden (hoofdstuk 8).

Inderdaad bleek dat consumptie wan rattenvoer dat $37 \%$ vertakte keten aminozuren bevat, in vergelijking met hel controlevoer, dat $25 \%$ vertakte keten aminozuren bevat, uitstel van 48 uur in het ontstaan van kanker anorexie tot gevolg heeft. Verdere verrijking van het woer mot vertakte keten aminozuren tot een total van $47 \%$ leidide niet tot een verder vitstel in het ontstaan van kanker anorexie. Het mechanisme dat deze gedragsveranderingen induceert, is echter onbekend en zou aanleiding moeten zijn woor nader onderzoek.

Remming van het sleutelenzym in de synthese van serotonine, te wetem tryptophaanhydroxylase, kan worden bereikt door intracerebrale toediening van para-chlorophenylalanine. Toediening van deze stof bij tumordragende ratten resulteert, zoals beschreven in hooidstuk $\mathbf{9}$, in een significante reductie van hersenconcentraties van serotonine, terwijl een eveneens significant uitstel van 2 dagen in het ontstaan van kanker anorexie werd bereikt. De tumordragende dieren werden echter uiteindelijk toch anorectisch, hoewel het 5-hydroxyindolazijnzuur nog steeds verlaagd was. Deze bevinding suggereert dat serotonine niet specifiek is wanneer het gaat om modificeren van kanker anorexie.

Intracerebrale toediening van de serotonine receptor blokker methysergide aan tumordragende ratten leidde niet tot modificatie van het eetgedrag bij deze dieren, zoals is beschrewem in hoofdstuk 10. Ten aanzien van de reden woor het gebrek aaln effectiviteit van deze serotonine blokker werd gehypothetiseerd dat kanker anorexie voornamelijk door serotonine 1 receptoren wordt gemedieerd terwill methysergide een hoge affiniteit heeft voor serotonine 2 receptoren.

Hooldstuk 11 beschrijft dat selectieve serotonine depletie door intracerebrale toediening van het serotonine neurotoxicum 5,7-dihydroxytryptamine geen ontremming van het eetgedrag van tumordragende dieren tot gevolg heeft. Hoewel eenmaal werd beschreven dat toediening van dit neurotoxicum resulteert in vermeerdering van voedselinname en verhoging wan lichaamsgewicht bij ratten, konden aanvullende onderzoekingen deze resultaten niet bevestigen. Derhalve is de significantie van de afwezigheild van enig effect van dit neurotoxicum op het voedselinname gedrag bij kankerdragende ratten niet duidelijk. In hoofdstuk 12 worden de resultaten van chemische stimulatie van de paraventriculaire hypothalamus beschreven. De conclusie van deze experimenten is dat norepinephrine geinduceerd eten onveranderd is bij tumordragende dieren en dat manipulatie van het serotoninerge systeem op het niveau van de paraventriculaire hypothalamus ook niet tot andere effecten bij tumordragende ratten leidt in vergelijking met niet tumor dragende controledieren. Derhalve dragen deze resultaten bij tot de onzekerheid welke bestaat omtrent de rol van serotonine als mediator van kanker anorexie. 
Hoofdstuk 13 vat de resultaten uit de boven beschreven experimenten samen. De conclusie luidt dat manipulatie van het serotoninerge systeem niet leidt tof een consistente steun voor de gehypothetiseerde rol van serotonine in het ontstaan van kanker anorexie. De resultaten wan de catecholamine bepalingen in de experimenten in het derde deel leidden niet tot een aanwijsbare rol van deze neurotransmitters in hel ontstaan van kanker anorexie.

Het discussiehoofdstuk 14 probeert de gewonden indicaties voor veranderd tryptophaan metabolisme te verklaren en komt tot de conclusie, dat, waar er te weinig kennis bestaat omtrent de mechanismen die onder normale omstandigheden tryptophaan concentraties in het plasma reguleren, verdere research nodig is om de oorzaken van de tryptophaan afwijkingen op te sporen, en daarmee een oordeel te geven of dit een woor de kankersituatie specifieke afwijking is. Voorts worden de schijnbaar tegenstrijdige conclusies van de twee groepen experimenten in dit proefschrift ten alanzien van de gehypothetiseerde rol van serotonine in het ontstaan van kanker anorexie beschouwd. De algemene conclusie van deze beschouwing luidt dat serotonine een algemeen remmende werking zou kunnen uitoefenen over een groot aantal gemotiveerde gedragingen, waaronder voedingsgedrag. Bovenop dit fenomeen gesuperponeerd zouden andere mechanismen meer specifiek anorexie bij kanker kunnen veroorzaken. De meest recente research lijkt aan te geven dat het dopaminerge systeem tot die additionele mechanismen zou kunnen behoren.

Hoofdstuk 15 beschouwt zeer in het kort in hoeverre kanker anorexie bijdraagt in het ontstaan van kanker cachexie, de uitmergelingstoestand, die vaak zo kenmerkend is voor kanker en zo significant bijdraagt in de morbiditeit en mortaliteit van deze ziekte. Vooralsnog blijkt weinig bekend omtrent deze specifieke quantificering wan de rol wan kanker anorexie, maar de algemene veronderstelling lijkt gerechtvaardigd, dat herstel van normale regelmechanismen van eetgedrag aanpassing aan de verhoogde energie behoefte van de tumor gastheer mogelijk maakt, waardoor die bijdrage in het ontstaan van kanker cachexie wordt geneutraliseerd, welke afkomstig is van verminderde inname van energie. 


\section{References}

Ahlskog, J.E., Randall, P.K. and Hoebel, B.G. (1975).

Hypothalamic hyperphagia: dissociation trom hyperphagia following destruction of noradrenergic neurons.
Science $190, p .399$.

Anamd, B.K. and Brobeck, J.R. (1951).

Hypothallamic control of food intake in rats and cats. Yale J. Biol. Med, 24, p. 123

Anand, B.K., China, G.S., Sharma, K.N., Dua, S. and Singh, B. (1964).

Actwity of single newrons in the hypothalamic teeding centers: effect of glucose. Am. J. Physiol. 207. P. 1146.

A.mderson, H.L., Benevenga, N.J. and Harper, A.E. (1968).

Associations among food and protein intake, serine dehydratase, and plasma amino acids. Am, J. Physiol. 214. p. 1008.

Ashley, D. Y.M, and Anderson, G.H. (1975).

Corretation between the pliasma tryptophan to ineutral anino acid ratio and protein intake in the self-selectimg weanling rat. $\downarrow$. Nutr. 105. p. 1412.

Balille, P., Millar, F.K. and Pratt, A.W. (1965).

Food and water intakes and Walker tumor growth in rats with hypothalamic lesions. Arn. I. Physiol. 209, p. 293.

Barrett, A.M. and McSharry, L. (1975).

Inhibition of drug-induced anorexia in rats by methysergide. ل. Pharm. Pharmac. 27, p. 889.

Baumgarten, H.G Gictor, S.J. and Lovenberg, W. (1973a).

Effect of intraventricular injection of 5,7 -dihydroxytryptamine on regional tryptophan hydroxylase of rat brain. J. Neurochem. 21, p. 251.

Baumgarten, H.G., Björklund, A., Lachenmayer, L. and Nobin, A. (1973b).

Evaluation of the effects af 5,7 -dihyidroxytryptamine an serotonin and catechallamine meurons in the rat CNS Acta Physiol Scand. 391, p. 3.

Baumgarten, H.G. and Björklund, A. (1976).

Neurotoxic indoleamines and monoamine neurons. Ann. Rew. Pharmacol. Toxicol, 16, p. 101.

Baxter, M.G., Miller, A.A. and Soroko, F.E. (1970).

The elfect of cyproheptadine on food consumption in the fasted rat. Br. J. Pharmacol. 39, p. 229.

Bender, D.A. (1978).

Regulation of 5-hycroxytryptamine synthesis. Proc. Nutr. Soc. $37, p .359$.

Bennet, J.P. Jir. and Snyder, S.H. (1978).

Serotonin synaptic receptors in the mammalian central nervows system. Fad. Proc. 37, p. 137.

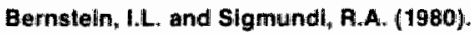

Tumor anorexia: a learned food aversion? Science 209 , p. 416

Blackburn, G.L., Moldawer, L.L. Usul, S., Bothe, A. Ji", OKeofe, S.J.D., Bistriam, B.F. (1979).

Branched chain amino acid administration and metabolism during starwation, injury, and infection. Supgery B6, $p$. 307 .

Bloxam, D.L. and Warren, W.H. (1974).

Errof in the determination of tryptophan by the method of Dencla and Dewey: A revised procedure.

Anal. Biochem, 60, p. 621.

Bitundell, J.E., Latham, C.J. and Leshem, M.B. (1973)

Biphasic action of a 5-hydroxytryptamine inhibitor on fentluramine-induced anorexia. J. Pharm. Fharmac. 25 , p. 492.

Blundell, J.E. and Leshern, M.B. (1975).

The effect of 5-hydroxytryptophan on tood intake and on the anomexts action of amphetamine and fentluramine.

J. Pharm. Pharmac. 27, p. 31. 
Blundetil, J.E and Latham, C.J. (1979).

Serotonergic infuences on food intake: elfect of 5 -hydroxylyptophan on parameters af ieeding behaviour in deprived

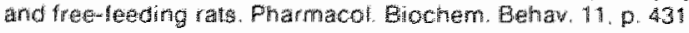

Bodartaky, $0 .(1975)$.

Blochemigtry of humam cancer. Academic Press. New York San Francisco. London

Breese, G.R. and Cosper, B.R. (1975)

Behaworal and biochemical interactions of 5,7 dihydroxytryptamine with various drugs when administered intracisternally to adult and developing rats. Brain Fes. 98, p. 597.

Brelach, S.T., Zemllan, F.P. and Howebel, B.G. (41976).

Hyperphagia and obesity following serotomir depletion by intraventicular p-chlorophemylalianine. Science 192. . 382

Brennan, M.F. (1977).

Uncomplicated starvation versus cancer cachexia. Cancer Res. 37 , p. 2359

Brennan: M.F. (1981).

Tatal parenteral nutrition in the cancer patrent. W. Engl. J Med. 305. p. 375.

Brobeck, J.R., Teppermian, d, and Long, C.N.H. (1943).

Experimental hypothalamic hyperphagia in the albino rat. Yate d. Eiol. 15. p. B3it.

Buse, M.G, and Reld, S.S. (1975).

A possible regulator of protein turnower in muscle. J. Cin. Invest. $56, p$. 1250.

Cllneschmidt, B.V., MeGutlin, J.C. and Werner, A.B. (1974).

Role of monoamines in the anorexigenic actions of fenfluramine, amphetamine and p-chlloromethamphetamine Eur J Pharmacol. 27, p. 313,

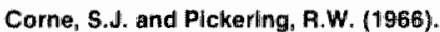

A posisible corretation between drug-induced hallucinations in man and a behawioural response in mice Psychopharmacologia (Berl.) 11, p. 65.

Coscina, D. V., Daniel, J. and Warsich, J.J. (1978a).

Potential non-serotonergic basis of hyperphagia elicited by intraventricular p-chlorophenylalanine.

Pharmacol. Biochem. Behaw. 9. p. 791.

Coscina, D.V. (1978b).

Effects of central 5,7 -dihydroxytryptamine on the medial hypathalamic syndrome in rats.

Ann. Wr Acad. Sci. 305, p. 627 .

Cummings, M.G., James, J.H., Soeters, P.B., Keane, J.M., Foster, J. and Fischer, J.E. (1976).

Fegional brain study of indoleamine melabolism in the rat in acute thepatic failure. J. Neurochem. 27, $p .74$.

Curzon, G. and Green, A.A. (1970).

Rapic method for the determination of 5-hydroxylryptamine and 5-hydroxyindaleacetic acid in small regions of rat brain. Br. S. Pflarmacol 39, p. 653 .

Curzon, $G_{n}$, Joseph, M.H. and Knott, P.J. (1972).

Elfects of immobilization and lood deprivation on rat brain tryptophan metabolism. J. Neurochem. 19, p. 1967 .

Curzan, $G_{n}$, Frledel, J. and Knott, P.J. (1973).

The eflect of latty acids on the binding of tryptophan to plasma protein. Nature $242, \mathrm{p} .498$

Curzon, G. and Knott, P.Ju, (1975).

Rapid elfecis of environmental disturbance on rat plasma unesterified tatty acid and tryptophan concentrattions and their prevention by antilipolytic drugs. Br. J. Pharmac. 54, p. 389.

Curzon, G. (1978).

Influefuce of nutritional state on transmitter symthesis. Proc. Nutr. Soc. 37, p. 155.

Dahliström, A. and Fuxe, K. (1965).

Evidence tor the existence of monoamine containing neurons in the central nervous system. Demonstration of monoamines in the cell bodies of brain stem neurons. Acta Physiol Scand. 62. suppl. 232. p. 1.

Della-Fera, M.A. and gille, C.A. (1979).

Cholecystokinin octapeptida: continuous picomole injections into the cerebral ventricles of sheep suppress leeding. Science 206, p. 471.

Deutsch, ل.A., Young, W.G. and Kalogerls, T.J., (1978).

The stomach signals satiely. Science 201, p. 165. 
DeWys, W.D. and Pascucci-Cimino, (1978).

Arrorexia, taste changes, and dief in cancer Compr. Ther, 4, p. 7 .

Dlonigi, R., Dominlonil. L. and Campani, M. (1980).

Infections in cancer patients. Surg. Clin. North Am, 60 . p 145 .

Doumas, B.T., Watson, W.A. and Biggs, H.G. (1971).

Albumin standards and the measurement of serum albumin with bromcresol green. Ohin. Chin. Acha 3. p. or

Edstrơm, S. (1980).

Giucose metabolism in cancer disease. A sutuy with special reference to the development of cancer cachexia

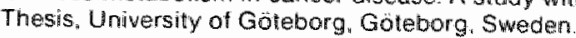

Ekman, L. Karlbergi, I., Edström, S., Scherstén, T. and Lundhoim, K. (1981).

Energy cost for inappropriately high protein synthesis in non growing sarcoma bearing mice.

Abstract EORTC symposium on Nutrition of the cancer patient. Brussets. Wan. 8-9.

Enna, S.J. (1981).

Brain Serotonin receptors and neuropsychiatric disorders in: Serotonin, current aspecis of neurochemistry and function, Haber, Gabay, Issidorides, Alivisatos, Eds. Adv, in Exp. and Med. Biol. Vol. 133. Plenum Press, London, New rork.

Epsitein, A.N. and Teitelbaum, P. (1967).

Splecific loss of the hypoglycemic control of feeding in recovered lateral rats. Am. J. Physiof. 213, p. 1159.

Felig. P. (1973),

Progress in endocrinology and metabolism. The glucose-alanine cycle. Metabolism 22, p. 179

Fenninger, L.D. and Mider, G.B. (1954),

Energy and in ltrogen metabolism in cancer. Adv. Cancer Fes. 2, p. 229.

Fernistrom, J.D. and Wurtman, R.J. (1971).

Brain serotonin content: increase following ingestion of carbohydrate diet Scilence $174, p .1023$.

Ferinstrom, J.D. and Wurtman, R.J. (1972a).

Brain serotonin content: physiological regulation by plasma neutral amino acids. Science 178, 0.414.

Fernstrom, J.D. and Wurtman, R.J. (1972b).

Elevation of plasma tryptophan by insulin in rat. Metabolism 21, p. 337.

Fernstrom, J.D. and Faller, D.V. (1978).

Neutral amino acids in the brain: changes in response to food ingestion. J. Neurocthem. 30, p. 1531

Fernstrom, J.D. (1979).

Diet-induced changes in plasma amino acid pattern effects on the brain uptake of large neutral amino acids, and on brain serotonin synthesis. J. Nieural Transm. Suppl. 15, p. 55.

Feinstrom, J.D. (1981).

Dietary precursors and brain neurotransmitter tormation. Ann. Rew. Med. 32, p. 413

Fleming D.G. (1969).

food intake studies in parabiotic rais. Ann. NY Acad. Sci. 157, p. 985

Freund, H.R., James, J.H. and Fischer, J.E. (1981).

Nitrogen-sparing mechanisms of singly administered branched-chain amino acids in the irjulurad rat.

Surquery 90 . P. 237.

Frledman , P.A., Kappelman, A.H. and Kaufman, S. (1972).

Partial purification and characterization of tryptophan hydroxylase from rabbit hindbrain. Wiol. Chem. 247, 0.4165

Fulks, A.M., LI, J.B. and Goldberg, A.L.. (1975).

Effects of insulini, glucase, and amino acids on protein turnover in rat diaphragm. U. Biol. Chem. 250, p. 290.

Fuller, R.W. and Wong, D.T. (1977).

Inhibition of serotonin reuptake. Fed. Proc. $36_{n}$ p. 2154

Fuller, P.W. (1980).

Pharmacology of central serotonin neurons. Arn. Rev. Pharmacol. Toxicol. 20, p. 111.

Funderburk, W.H., Hazelwood, J.C., Ruckart, R. T. and Ward, J.W. (197I).

is 5-hydroxytryptamine involved in the mechanism of action of fenturamine? J. Pharm. Pharmac. $23, \mathrm{p} .468$ 


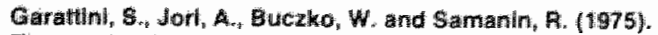

The mechanism of action of terthuramine. Pastgrad. Med J 51 (Suppl. 1). p. 27

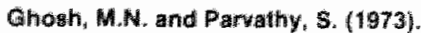

The effect of cyproheptadine on water and food intake and on body weight in the lasted adult and weanling rats Br. J. Phamacol. 48 , p. 328.

Gillbbs, J., Yowing, A.C. and Smith, G.P. (1973).

Cholecystokintn decreases tood intake in rats J. Comp. Physiol. Psychol, 84, p. 488.

Glowinski, $d$. and twergen, L.L., (1966).

Regional studies ot catecholamines in the rat brain - 1. N. Neurochem. $13,0,655$

Goldman, H.W., Lehr, D. and Fritedman, E. (1971).

Antagonistic gffects of alpha and beta-adrenergically coded hypothalamic neurons on consummatory behaviour in the rat. Nature $231, p, 453$.

Goudle, A, $\mathrm{J}_{3}$, Thoirnton, E.W. and Wheeler, T.J. (1976).

Effects of Lilly 110140 , a specific inhibitor of 5-hydroxyryptamine uptake, on food intake and on 5-hydroxylryptophaninduced anorexila. Evidence for serotoninergic inhibition of feeding. J. Pharm. Pharmac, 28, p, 318.

Grant ${ }_{n}$ J.P. and Weills, S.A. (1974).

Tumor resistance in rats immunized lo tetal tissues. J. Surg. Res. 16, p. 533.

Crossman, S.P. (1960).

Eating or drinking elicited by direct adrenergic or cholinergic stimulation of hypothalamus. Science 132, 0.301.

Grosgman ${ }_{H}$ S.P. (1962).

Direct adrenergic and cholinergic stimulation of hypothalamic machanisms. Am. J. Physiol. 202 (5), p. 872 .

Girossman, S.P. (1980).

The ineuroanatomy of eating arad drinking behavior. Chapter 14 in: Neuroendocrinology. Eds. Krieger, D. and Hughes, J.C.; Sinauer Associates Inc., Publ., Sunderland, Massachusetts, 1980

Halgler, H.J. and Aghajanian, G.K. (1977).

Serotonin receptors in the brall. Fed. Froc. 36, p. 2159.

Harper, A.E. and Rogers, Q.R. (1965).

Amino acid imbalance. Proc. Nutr. Soc. 24, p. 173.

Heffner, T.G., Hartman, J.A., Selden, L.S. (1980).

Fegding increases dopamine metabolism in the rat brain. Sicience 208, p. 1168.

Hett, F., Melamed, E. and Wurtman, R.J. (1980).

Partial lesions of the dopaminergic nigrostratal system in rat brain. biochemical characterization.

Brain Res. 195, p. 123.

Hetheringlon, A.W. and Aamson, S.W. (1940).

Hypothalamic lesions and adiposity in the rat. Anat. Aec, 78, p. 149.

Hoebel, B.G.,Zemlan, F.P., Trulson, M.E, MacKenzle, R. G., DuCret, R.P. and Noreill, C. (1978).

Differential elfects of $\mathrm{p}$-chlorophenylatanine and 5,7 -dihydraxytryptamine on feeding in rats.

Ann. NY Acad. Scl. 305, p. 590 .

Jeppason, B., Gimmon, Z. James, H.J. and Fischer, J.E. (1980a).

Changes in plasma and brain amino acids in acute respiratory and metabolic acidosis. Surg. Forum $3 \%_{\text {" }} p .218$.

Jeppsson, B. Freund "H.F., Gimmon, Z."James, J.H., Von Mleyenleldt, M.F. and Fischer, J.E., (1980b).

Blood-brain barrier derangement in sepsis: cause of septic enceplnalopathy? Am. J. Surg. 141, p. 136.

Jappsson, . Frewind, H.A., Gimman, Z., James, J.H., Von Meyenfeld, M.F. and Fischer, J.E.

Slood-brain batrier derangement in uremic encephalopathy. Submitted for publication in Metabolism, 1982.

Jequler, E., Loventberg, W. and Sjoerdsma, A. (1967).

Tryptophan hydroxylase inhibition: the mechanism by which p-chlorophenylalanine depletes rat brain serotonin.

Mol. Pharmacol. 3. p. 274

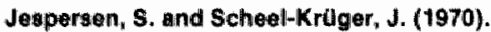

Antagonism by methysergide of the 5-hydroxytryptamine-like action of toxic dloses of fenfluramine in dogs.

J. Pharm. Pharmas. 22, p. 637 . 


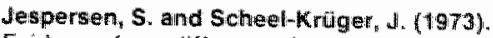

Evidence for a difference in mechanism of action between fenturamine-and amphetamine-induced anorexis.

J. Phalm. Pharmac. 25, p. 49.

Kantak, K.M., Wayner, M.J. and Stein, J.M. (1978).

Effects of various periods of food depivation on serotonin synthesis in the lateral hypothalamus

Pharmac Biochem. Behaw 9. p. 535 .

Karlberg, !. (1980).

Cancer cachexia, the role of anorexia and cell proliferation. Thesis, Universily of Götaborg. Goteborg. Sweden.

Karlberg, II., v. Lammeren, F.M. and Fischer, J.E. (1982).

Anorexia decreases skeletal musclle protein synthesis in cancer cachexia

Abstract 4th ESPEN Congress. Clinical Nutrition 1. Spec. Suppi, p. 131.

Kawtamura, 1., Moldawer, L.L., Bothe, A., Bistrian, B.R. and Blackburn, G.L.

Effects of branched cham amino acid enriched hyperalmentation an proten dynamics in tumor bearing rats.

Abstract ASPEN 6th Clinical congress febt. 3-6, 1982, San Francisco.

Kirsch, R.E., Saunders, S.J., Firth, L., Wheht, S, Kelman, L, and Brock, J.F. (1969).

Plasma amino acid concentration and the regulation of albumin symthesis. Am. J. Cin. Nuin. 22. p. 1559.

Knott, P.ل. and Curzan, G. (1974).

Elfect of increased rat brain tryptophan on 5-hydroxytryptamine and 5-hydroxyindolyl acetic acid in the hypothalamus and other brain regions. N. Neurochem. $22, \mathrm{p}$. 1065 .

Koe, B.K. and Weilssman, A. (1966)

p-Chlorophenylaianine: a specitic depletor of brain serotonin. J. Pharmacol. Exp. Ther. 15t, p. 499.

Krause, R., James, J.H., Zlparo, V. and Fischer, U.E. (1979a).

Brain tryplophan and the neoplastic anorexia-cachexia syndrome. Cancer 44, p. 1003.

Krause, R., James, H., Humphrey, Chr. and Fischer, J.E. (1979tb).

Plasma and brain amino acids in Waiker 256 carcino sarcoma bearing rats. Cancer Fes. 39, p. 3065

Krause, A. $_{\text {(1980). }}$.

Anorexia in cancer. Thesis, Uniw, of Limburg. Maastricht, The Netherlands.

Krleger, D.T., Crowley, W.R., O Donohue, T.L. and Jacobowitz, D.M. (1980).

Effects of food restriction an the periodicity of carlicosteroids in plasma and on monoamine concentrations in discrete brain nuclei. Brain Res. 188, p. 167.

Kruk, Z.L. (1973).

Dopamine and 5-hydroxytryptamine inhibit feeding in rats. Nature New Biol 246, p. 52.

Lammeren v., F.M., Chance, W.T. and Fischer, J.E. (1982a).

Unpublished observations.

Lammeren W. F.M., Chance, W.T., Karlberg, I, and Fischer, J.E. (1982b).

Supraoptimal enteral fexeding normalizes increased brain indoleamine metabolism associaled with cancer anorexia.

Abstract 4th ESPEN Congress, Chinical Nutrition 1, Spec. Suppl, p 130

Lammeren v., F.M., Kariberg, I, Vorhees, M., Chen, M.H., Chance, W.T, Von Meyenteldit, M.F., Jidfle, J.N. and Mutrphy, R.F. (1982C).

The effect of tumor burder on lood intake and circulating immu no reactivities of glucagon and insulin in anonectic fats. Abstract, 4th Intemational Symposion Gastrointestinal Horm. Stockholm, Sweden, 20-23 Junt, 1982.

Lammeren Y., F.M., Chance, W.T., Karlberg, U. and Fischer, J.E. (1982d).

Normalization of brain indoleamines in anorectic tumor bearing rats by forced enteral fieeding

Soc Neurasci. Abstr, 8. p. 606.

Latham, C.J. and Blundelli, J.E. (1979).

Evidence for the effect of tryptophan on the pattern of tood consumption in free feeding and food deprived rats Eife Sci. 24. p. 1971.

Lehr, D, and Goldman, W. (1973).

Continued pharmacologic analysis of consemmatory behavior in the albino rat. Eur. J. Pharmacol. 23, p. 197

Lelbowitz, S.F. (1976).

Brain catecholaminergic mechanisms for control of hunger. In: Hunger: basic mechanisms and chlinical implications ed. by Novin, D., Wyrwicka, W. and Bray, G. Riaven Press, New Vork. 
Latbowitz, S.F. (1978)

Par aventricular nuclidus a primary site medianing ad rengrgic stimulation of leeding and orinking. Pharmacol. Biochem Behay. B. p. 163

Lelbowitz, S.F. (1978b)

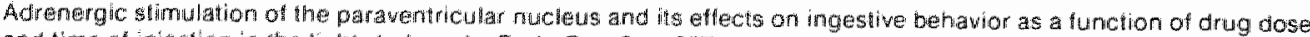
and time of injection in the light-dark cycle Brain Res. 3. p. 357

Lelbowitu, S,F, and Papadakois, P.d. (1978c).

Serotonin-ronepinephthe interaction in the paraventricular nucleus: antagonstic efects on feeding behawion the the rat. Soc. Neuroset. Abstr. 4, p. 177

Leung, P.M-B. and Pogers, O.R. (1969).

Food intake regulation by plasma amino acid pattern. Lite Sci. 8. p. 1.

Llebelt, A., A, Liebelt, A. G, and Johnston, H.M. (197i).

Lipid mobitization and food intatke fli experimentally obese mice bearing transpllanted umors

Proc Soc. Exp. Biol Med. 138, p. 482

Lindmarlk, L., Ekman, L., Edsitröm, S. Kartberg, I. and Lumdholm, K. (1982).

Adaptive changes in whole body energy metabolism in experimental cancer

Aibstract 4th ESPEN Congress, Clinical Nutrition 1. Spec. Suppt. p. 134

Lorens, S.A. ("⿻1978).

Some behavioral ellects of serotonin depletion depend on method a comparison of 5.7-dihydrowytryptamine pwohlorophenylatanine, p-chloroamphelamire and electrolytic raphe lesions. Ann. NY Acad. Sci. 305, p. 532.

Lundholm, K., Karlberg, I. andi Scherstên, T. (1978a).

Studies on brosiynthesis of albumin and hepatic proteins in cancer patients. Eur. J. Clin. Invest. 8, p. 331.

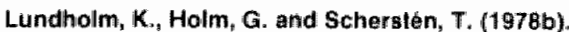

Insulin resistance in patients with cancer. Cancer Res. 38 , p. 4665

Lundhollm, K, Ekman, L., Edstrom, $S_{,}$Karlberg, l., Jagenburg, R. and Schersten, T. (1979).

Protein synthesis in liver tissue under the influence of a methylcholanthrene-induced sarcoma in mice.

Cancer Fes. 39. p. 4657

Lundho\#m, $K_{\text {, }}$ Edström, $S_{n,}$ Karlberg, L., Ekman, L. and Scherstén, T. (1980).

Relationship of food intake, body composition, and tumor growth to host metabolism in non growing mice with sarcoma. Cancer Ales. 40, p. 2516

Lundholm, K. (1981).

Cachexia metabolism. Abstract EORTC Symposium on Nutrition of the cancer patient. Brussels Jan. 8-9.

Mabry, P.D. and Campbell, B.A. (1973).

Serotonergic inhibition of catecholamine-induced behavioral arousal. Brain Res, 49, p. 381.

Mackenzie, A.G., Hoebel, B.G., Ducret, F.P. and Truison, M.E. (1979),

Hyperphagia following intraweritricular p-chlorophenylalanine-, leusine- ar Iryptophan-methyl esters: lack of correlation with whole brain serotonin lowels. Pharmacol. Biochem. Behav. 10, p. 951

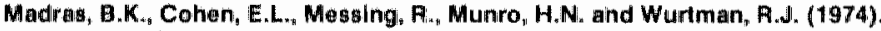

Relovance of free tryptophan in serum to tissue tryptophan concentrations. Metabolism 23, p. 1107

Margules, D.L. Lewlis, M.d., Dragovich, d.A. and Margules, A.S. (1972).

Hypothalamic norep mephrine: circadian rhythms and the control of feeding behavior. Science 178, p. 640.

Marshall, J.F., Richardison, J.s., and Teltelbaum, P. (1974),

Nigirostriatal bundle damage and the lateral hypothallamic symdrome. J. Comp. Physiol. Psychol. 87, p. 80B.

Martyama, Y., Oshima, T. and Naka]ima, E. (1980).

Simultaneous determination of catecholamines in rat brain by reversed-phase liquid chromatography with electrochemical idetection. Life Sci. 26, p. 1115

Mawson, $C_{\text {. }}$ and Whittington, $H .(1970)$.

Evaluation of the peripheral and central antagonistic activities against 5-hydroxytryptamine of some new agents Br. u. Pharmiacol. 39, p. 223.

Mayer, J. (1955).

Regulation of energy intake and the body weight: the ghucostatic theory and the lipostatic hypothesis

Ann. NY Acad. Sci. 63 , p. 15 
McCaleb, M.L. and Myers, R.D. (1980).

Cholecystokinin acts on the hypothatamic noradrenergic system inwolved in teecting. Peptides 1. p. 4h"

McMenamy, R.H. and Oncley, $\mathbb{H}$. L. (1958).

The specific binding of I-tryptophan 10 serum albumin. J. Biol. Chem. 233, p. 1436.

McMenamy, R.H., Lund, C.C., wan Marcke, J. and Oncley, J.L. (1961).

The binding of lltyptophan in human plasma at $37^{\circ} \mathrm{C}$. Arch. Blochem. Bophys 93, p. 135.

Melamed, $E_{n y}$ Hefti, F, and Wurtman, $R$.J. (1980).

Tyrosine administration increases striatal dopamine release in rats with partial nigrositrialal lesions.

Proc. Natl Acad. Sci USA 77 . p. 4305.

Mider, G.B., Testuk, H. and Morton, J.J. (1948).

Effects of Walker carcinoma 256 on lood intake, body weight and nitrogen metabolism of growing rats.

Acta Union Int. Contra Cancrum 6. p. 409.

Morrison, S.D. (1968).

Effect of growth of a tumor on the regulation of water intake. Jl. Nat. Cancer Inst. 41, p. 1241.

Mormison, S.D. (1973).

Limited capacity for motor activity as a cause for declining food intake in cancer. J. Nat. Cancer inst. 54. p. 1535.

Morrison, S.D. (1975).

Origins of nutritional imbalance in cancer. Cancer Res. 35, p. "3339.

Morrison, S.D. (1976a).

Geneiration and compensation of the cancer cachectic process by spontaneous modification of feeding behavion.

Cancer Res. 36. P. 228.

Morrison, S.D. (1976b).

Theoretical review; control of food intake in cancer cachexia: a challenge and a tool. Physiol. Behav, 17, p. 705

Morrison, S.D. (1978).

Origins of anorexia in neoplastic disease. Am. J. Clin. Nutr. 31, p. 1104.

Munro, H.N. and Young, V.F. (1977).

3- Methylhistidine as an index of rate of muscle protein breakdown. In: Kinische Anasthesiologie und

Intensivtherapie. Eds. Ahneteld, F.W. Bergman, H., Burri, C. and Dick, W., vol, 13, p. 35. Springer Verlag. New York.

Myers, R.D. (1978).

Hypothalamic actions of 5-hydroxytryptamine neurotoxins: feeding, drinking, and body temperature.

Ann. NY Acad Sici. 305, p 556.

Nishizawa, N., Noguchi, T., Hareyama, S. and Funablkt, R. (1977).

Fractional flux rates of N-methylhistidine in skin and gastrointestine: the contribution of these tissues to urinary excretion of $\mathrm{N}$-methy/histidine in the rat. Br. J. Nutr. $38, \mathrm{p} .149$.

Norton, J.A., Shamberger, Riv, Stein, T.P., Milne, G.W.A. and Brennan, M.F. (1981)).

The influence of tuimor-bearing on protein metabolism in the rat. $J$. Surg. Pes. 30. p. 456

Odessey, R., Khalirallah, E.A. and Goldberg, A.L. (1974),

Origin and possible signiticance of alanine production by skeletal muscle. J. Bioll. Chem. 249, p. 7623

Oldendort, W.H. (1971).

Brain uptake of radiolabeled amino acids amines, and hexoses after arterial injection, Am. J. Physiol. 221, p. 4629

Oomura, Y. (1976) .

Sigmificance of glucose, irsulin, and free fatty acid on the hypothalamic feeding and satiety neurons. In: Hunger: besic mechanisms and clinical implications, ed by Novin. D. Wyrwicka, W. and Bray, G. Raven Press, New York

Osborne, T.B. and Mendel, L.B. (1916a).

The amino-acd minimum for maintenance and growth, as exemplified by further experiments with lys ine and tryptophane. J. Biol Chern. 25, f. 1.

Osboirne, T.B. and Mendel, L.B. (1916b).

A quantitative comparison of casein, lactalburnin, and edestin for growth or maintenance, J. Biol. Chem. 26. p. 1

Panksepp, لlu, Bishop, P. and Rossi, J.llll (1979).

Neurohumoral and eindocrine control of feeding. Psychoneuroendocrinology t, p. 89. 
Pardifidge; W. W. (1977):

Kinefics of compet the inhibition of rewtrat amino acid transport across the blood-brain barter.

J. Nasurothem. 28. p. 103

Parditidge, W.M. (1979a).

Tryptophan lransport through the blood-bram barmer. in wivo measurement of free and abumin-bound amino acio. Lite Sci. 25 , p. 1519

Pardridge, W.M. (1979b).

The role of blood-bram barrier transpont on tryptophan and other neutral amino acids in the regulation of substratelimited pathways of brain amino acid metabolism. J. Neural Transm. Suppl. 15, p. 43.

Pellegrino, L.J. and Cushmain, A.J. (1967).

A stereotaxio allas of the rat brain. New York. Appleton-Century-Crofs.

Pérez-Cruet, J, Tagllamonte, A., Tagllamonte, P. and Gessa, G.L. (1972).

Changes in braily serotonirs metabolism assochated with fasting and satiation in rats. Life Sci. 11, p. 31

Perez-Cruet, d, Chase, T.N. and Murphy, D.L. (1974).

Diatary regutathon of brain tryplophan metabolism by plasma ratio of tree tryptophan and neutral amino acids in humans. Vature 248, p. 693 .

Peroutka, S.J. and Snycler, S.H. (1979).

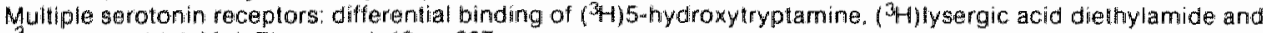
$\left({ }^{3} \mathrm{H}\right)$ spiroperidol Mot. Pharmacol. 16, p. 387

Percutka, S.d., Lebovitz, R.M. and Snyder S.H. (1981),

Two distinct central serotonin receptors with different physiological functions. Science 212, P. 827

Popp, M.B., Morrison, S.D. and Brenman, M.F. (1981)

Tolal parenteral nutrition in a methylcholanthrene-induced rat sarcome model.

Cancer Treat. Rep. 65 (Suppl. 5) p. 137.

Prath A.W. and Putney, F.K. (1958).

Observations on the energy metabolism of rats receiving Walker tumor 256 transpllants. J. Nat. Cancer $\mid$ inst. 20 . p. 173 .

Rodwell, V.W. (1977).

Catabolisim of amino acids. In: Review of physiological chemistry, 16th edition. Eds.: Harper, H.A. Fodwell, V.W. and

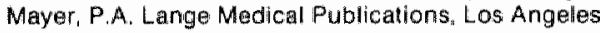

Rogers, O.R. and Leung, P.M.B. (1973).

The influence of amino acids on the neuroregulation of food intake. Fed. Proc. 32, p. 1709

Rose, W.C. (1931).

Feeding experiments with mixtures of highly purified amino acids. J. Biot. Chem. 74, p. 155.

Rogen, H.M. Yoshimura, N., Hodgman, J.M. and Flscher, J.E. (1977),

Plasma amino acid patlarns in hepatic encephalopathy of differing etiology. Gastroenteroliogy 72, p. 483

Saller, C.F. and Stricker, E.M. (1976).

Hyperphagia and increased growth th rats alter intraventricular injection of 5,7 -dihydroxytryptamine Sclence 192 , p. 365 .

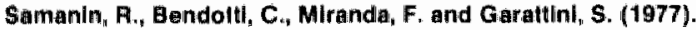

Decrease of food intake by quipazine in the rat relation to serotoninergic receptor stimulation.

J. Pharm. Pharmac, $29,0.53$

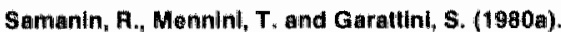

Evidence that it is possible to cause anorexia by increasing release and/or directly stimulatimg postsynaptic serotonin receptors in the brain. Prog. Neuromsychopharmacol. 4. p. 363.

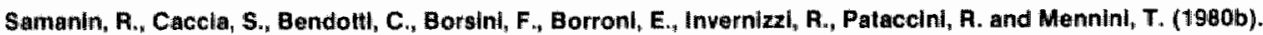
Further studies on the mechanism of serotonln-dependent anorexia in rats. Psychopharmacalogy 68, p. 99.

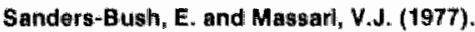

Actions of drugs that deplete serotonin. Fad. Proc. $36, p .2149$.

Sharma, K.NN . Anand, B.K., Dua, S and Baldev Singh (1961).

Fole of stomact in regulation of activities of hypothatamic feeding centers. Am. A. Physiol. 201, p. 593.

Sherman, C.D. Jir, Morton, J.J. and Mlder, G.B. (1950).

Potential sources of tumor nitrogen. Cancer Res. 10, p. 374 
Singer, G., Sanghwi, I. and Gershon, S. (1971).

Exploration of certain behaworal patterns induced by psychoactive agents in the rat. Commum. Behav. Biol. 6. p. 3at.

Stevensom, J.A.F., Box, B.M. and Wright, R.B. (1963)"

The effect of a cold enviromment on malignant anorexia. Can. J. Biochem. Physiol. 41.p.531.

Strominger, J.L. and Brobeclik, J.R. (1953).

A mechanism of regulation of food intake. Yale d. Biol. Med. $25,0.383$

Soeters; P.B. (1979).

Hepatic enceps;

Theologides, A. (1974).

The anorexia-cachexia sy ndrome; a new hypothesis. Ann. NY Aciad. Sci. 230, p. 14.

Theologlides, A. (1976).

Anorexla-producing intermediary metabolites. Am. J. Clin. Nutr. 29, p. 552.

Ungerstedt, $U_{n}(1971)$.

Stereotaxic mapping of the monoamine pathways in the rat brain. Acta Physial. Scandinat. Suppli. 367, p. If

Warnold $I_{1}$, Lundholm, $K_{\text {. }}$ and Scherstên $n_{n} T$. (1978).

Energy balance and bordy composition in cancer patients. Cencer Res. 38, p. 1801.

Wooley, O.W., Wooley, S.C. and Woods, W.A. (1975).

Effect of calories on appetite for palatable food in cbese and nonobese humans.

J. Comp. Physiol. Psychol. 89 (6), p. 619

Wurtman, A.J., Larim, F., Mostafapour, S. and Fernstrom, J.D. (1974).

Brain catechol synthesis: control by brain tyrosine concentration. Science 185, p. 183.

Wurtman, Ri.J. (1979).

When - and why - should nutritional state contral neurotransmitter synthesis? J. Neurali Transm. Suppl. $15, p .69$.

Yaksh, T.L. and Audy, T.A. (1976).

Chronic catheterization of the spinal subarachnoid space. Physiol Behav. 17, p. 1031.

Young, S.N., Lal, S., Feldmuiler, F., Sourkes, T.L., Ford, R.M., Kiely, M. and Martín, J.E. (1976).

Paraltel variation of ventricular CSF tryptophan and free serum tryptophan in man.

J. Neurol. Neurosurg. Psychiatry 39, p.6.1.

Young, S.N. and Lal, S. $(1980)$.

CNS tryptamine metabollism in hepatic coma. J. Neuratransmission $47, p .153$.

Young, V.R., Alexis, S.D., Baliga, B.S. and Munro, H.N. (1972).

Metabolism of administered 3-methylhistidine. J. Biol. Chem. 247, p. 3592

Yuwlier, A., Oldendort, W.H., Gieller, E. and Braun, L. (1977).

Effect of albumin binding and amimo acid competition on tryptophan uptake into birain. J. Neurochem. $28,0.1015$ 



\section{Acknowledgements}

Many people have supported me in the activities which resulted in publication of this thesis. It is impossible to thank everyone in person for their great help, but I want to acknowledge specifically the contributions of those, without whom these activities would never have started, let alone finished.

Prot. Co Greep, your dynamic personality created the infrastructure of a Department of Surgery that gave me the opportunity to spend 23 months in Cincinnati and work on the subject of this thesis. It guarantees also the special productive climate in which a resident receives his surgical training in your department. I am deeply grateful for your encouragement and guidance.

Prof. Joe Fischer, I shall always remember the hospitality shown by the staff in your laboratory, department and hyperalimentation unit. You provided me with every opportunity to make my stay in Cincinnati a fruitful one. Without the help of you and your family not only $\|$ but also. Ellen, Suzanne and Erik would not have enjoyed the two years in Cincinnati as much as we did.

William Chance, your help proved essential in many experiments, that otherwise would not have been performed. Your fine teaching has been of great support to me on many occasions. I also want to acknowledge your help in reviewing the thesis, transforming my "English" into understandable language. Our collaboration meant a great deal to me, and has led to a warm friendship between us and our families.

Dr. Soeters, Prof. Struyker Boudier and Prof. Visser, II greatly appreciate your critical reading of the manuscript, resulting in remarks that I feel, added to the quality of this thesis Prof. Lemmens, you enabled me to start my surgical training in Maastricht.

Dr. Ruud Krause, the encouragement you gave me to continue in Cincinnati with the work you initiated in Boston, and the lengthy, stimulating discussions are of great value to me. Howard James, your technical advice and assistance, and Drs. Bengt Jeppsson,

Zvil Gimmon, Patrizia Cardelli-Cangiano, Carlo Canglano and Per Herlin, your collaboration and interesting discussions I remember with gratitude.

Laura Edwards, Christie Minnema, Mary Williams and William Brenner, I am thankful for your skilful technical assistance.

Laura, and later also Leo Strijbosch, your valuable help with the statistical analysis made interpretation of the results possible, while, Jean Loos, your charts illustrate beautifully these results.

Dr. Paul Jörning, your pushing helped me to put this text on paper.

Chrys Steegmans, I thank you for your fine cover design.

Marcia van Puljenbroek, I thank you very much for your patience, and skill in typing and changing the manuscript. Maartje Duyzings, " thank you for your assistance in secretarial work and Henk Jas, for your technical skill in processing the manuscript to the computerized typesetter in the printing office.

Finally I thank my collegues in the Department of Surgery of St. Annadal Hospital for taking over my duty when I needed time to work on this thesis.

Last but not least I want to mention Ellen, Suzanne and Erik, for their endurance and understanding, allowing me 'to stay in my corner'. We will soon make up for this. 



\section{Curriculum vitae}

Maarten Frederik von Meyenfeldt was born on January 26 th 1947 in Makassar, Netherlands Indies. He graduated from high school (Incrementum, Het Baarnsch Lyceum. Gymnasium B) in 1966. In the same year he went to Medical School at the University of Utrecht, and graduated in 1973. From January 1974 until June 1975 he served Her Majesty, Queen Juliana of the Netherlands in the military service. He started his surgical residency training at the Surgical Department of St. Barbara Hospital in Geleen (Heads Dr. J.J.H.M. Daniëls and $\mathrm{J}$. de Vries). In February 1976 his formal training started at the Surgical Department of St. Annadal Hospital, University of Limburg, Maastricht, first under supervision of Prof. Dr. H.A.J. Lemmens, later of Prof. Dr. J.M. Greep.

From January 1979 to December 1980 his surgical residency was interrupted by a clinical and research fellowship at the Department of Surgery and Surgical Physiology Laboratory of the University of Cincinnati Medical Center, Cincinnati, OHIO, U.S.A. (Head Prof. J.E. Fischer, M.D.). From December 1980 on his surgical residency program has been resumed at the Department of Surgery of St. Annadal Hospital, University of Limburg, Maastricht, The Netherlands. 